\title{
MULTILOOP ALGEBRAS, ITERATED LOOP ALGEBRAS AND EXTENDED AFFINE LIE ALGEBRAS OF NULLITY 2
}

\author{
BRUCE ALLISON, STEPHEN BERMAN, AND ARTURO PIANZOLA
}

\begin{abstract}
Let $\mathbb{M}_{n}$ be the class of all multiloop algebras of finite dimensional simple Lie algebras relative to $n$-tuples of commuting finite order automorphisms. It is a classical result that $\mathbb{M}_{1}$ is the class of all derived algebras modulo their centres of affine Kac-Moody Lie algebras. This combined with the Peterson-Kac conjugacy theorem for affine algebras results in a classification of the algebras in $\mathbb{M}_{1}$. In this paper, we classify the algebras in $\mathbb{M}_{2}$, and further determine the relationship between $\mathbb{M}_{2}$ and two other classes of Lie algebras: the class of all loop algebras of affine Lie algebras and the class of all extended affine Lie algebras of nullity 2 .
\end{abstract}

\section{INTRODUCTION}

Affine Kac-Moody algebras over an algebraically closed field of of characteristic 0 comprise one of the most widely studied and applied classes of infinite dimensional Lie algebras. Kac's realization theorem is of fundamental importance in this area as it provides an explicit construction of affine algebras from finite dimensional simple algebras. More precisely, the version of this theorem that we use states that the algebras of the form $\overline{\mathfrak{g}^{\prime}}:=\mathfrak{g}^{\prime} / Z\left(\mathfrak{g}^{\prime}\right)$, where $\mathfrak{g}$ is an affine algebra, are the same up to isomorphism as the loop algebras of the form $\mathrm{L}(\dot{\mathfrak{g}}, \sigma)$ where $\dot{\mathfrak{g}}$ is a finite dimensional simple and $\sigma$ a diagram automorphism of $\dot{\mathfrak{g}}$. (See Section 4.2 below for the definition of a loop algebra.)

Now finite dimensional simple algebras and affine algebras can be regarded as nullity 0 and nullity 1 Lie algebras, in the sense that the additive group generated by the isotropic roots is free of rank 0 or 1 respectively. Taking this point of view, it is irresistible to look for higher nullity analogues of these algebras, and to hope that loop algebras in some form will provide constructions of algebras of increasing nullity.

A considerable amount of interesting work has been done on higher nullity algebras in recent years using a number of different approaches. These approaches have included: axiomatic characterizations; realizations as multiloop algebras, iterated loop algebras, or matrix algebras over nonassociative coordinate algebras; constructions by means of generators and relations determined by root systems; constructions of representations; and the study of higher nullity algebras as forms

Date: Feb 11, 2010.

2000 Mathematics Subject Classification. 17B65, $17 \mathrm{~B} 67$.

Key words and phrases. loop algebras, multiloop algebras, extended affine Lie algebras.

B. Allison and A. Pianzola gratefully acknowledge the support of the Natural Sciences and Engineering Research Council of Canada. A. Pianzola gratefully acknowledges the support of CONICET. 
of untwisted loop algebras and their relation to torsors over Laurent polynomial rings.

In this paper, we will focus on three of these approaches (although some of the other approaches will also be mentioned), namely the construction of higher nullity algebras using multiloop algebras and iterated loop algebras, and the axiomatic description of higher nullity algebras. To outline our main results, we now introduce three classes of algebras $\mathbb{M}_{n}, \mathbb{I}_{n}$ and $\mathbb{E}_{n}$, which depend on a nonnegative integer $n$, and we say that the algebras in these classes have nullity $n$. In each case, we mention a sample of the papers which deal with these topics. There are many other interesting related articles, several of which are listed in the bibliography.

- Multiloop algebras and $\mathbb{M}_{n}$ [EMY, vdL, GP1, ABFP1, ABFP2, Na] There is a natural generalization of the loop construction that produces a multiloop algebra $\mathrm{L}(\mathfrak{g}, \boldsymbol{\sigma})$ from a Lie algebra $\mathfrak{g}$ using an $n$-tuple $\boldsymbol{\sigma}=$ $\left(\sigma_{1}, \ldots, \sigma_{n}\right)$ of commuting finite order automorphisms of $\mathfrak{g}$ (see Section 4.1 below). We denote by $\mathbb{M}_{n}$ the class of all Lie algebras that are isomorphic to multiloop algebras of the form $\mathrm{L}(\dot{\mathfrak{g}}, \boldsymbol{\sigma})$, where $\dot{\mathfrak{g}}$ is finite dimensional and simple and $\boldsymbol{\sigma}=\left(\sigma_{1}, \ldots, \sigma_{n}\right)$ is as indicated. By convention, $\mathbb{M}_{0}$ is the class of all finite dimensional simple Lie algebras.

- Iterated loop algebras and $\mathbb{I}_{n}[\mathrm{~W}, \mathrm{Po}, \mathrm{ABP} 1, \mathrm{ABP} 2, \mathrm{ABP} 2.5]$ Let $\mathbb{I}_{0}$ be the class of all finite dimensional simple Lie algebras; and, for $n \geq 1$, let $\mathbb{I}_{n}$ be the class of all Lie algebras that are isomorphic to loop algebras of the form $\mathrm{L}(\mathfrak{g}, \sigma)$, where $\mathfrak{g} \in \mathbb{I}_{n-1}$ and $\sigma$ is a finite order automorphism of $\mathfrak{g}$.

- EALAs and $\mathbb{E}_{n}$ [Sai, H-KT, BGK, AABGP, Neh2, Neh3] An extended affine Lie algebra, or EALA for short, is an algebra $\mathfrak{g}$ that satisfies a list of natural axioms that are modeled on well known properties of finite dimensional simple Lie algebras and affine algebras (see Section 5.1 below). One of the axioms requires that the rank of the group generated by the isotropic roots of $\mathfrak{g}$ be finite, and this rank is called the nullity of $\mathfrak{g}$. Further, the subalgebra $\mathfrak{g}_{c}$ of $\mathfrak{g}$ generated by the root spaces corresponding to nonisotropic roots is called the core of $\mathfrak{g}$. (In general $\mathfrak{g}_{c} \subseteq \mathfrak{g}^{\prime}$, and these algebras are equal in the affine case.) We denote by $\mathbb{E}_{n}$ the class of all algebras that are isomorphic to algebras of the form $\mathfrak{g}_{c} / Z\left(\mathfrak{g}_{c}\right)$ for some EALA $\mathfrak{g}$ of nullity $n$.

It is not difficult to show that $\mathbb{M}_{0}=\mathbb{I}_{0}=\mathbb{E}_{0}$, and that this is the class of finite dimensional simple Lie algebras (Proposition 5.4.3). Of course the classification of these algebras is well known. Also, we will see using Kac's realization theorem that $\mathbb{M}_{1}=\mathbb{I}_{1}=\mathbb{E}_{1}$ and that this is the class of derived algebras modulo their centres of affine algebras (Corollary 6.3.3). Moreover, the Peterson-Kac conjugacy theorem for affine algebras provides a classification of the algebras in $\mathbb{M}_{1}$ (Corollary 6.3.7). In general $\mathbb{M}_{n} \subseteq \mathbb{I}_{n}$ (see 4.4.2), but no other containments hold, even for $n=2$.

This paper concludes a sequence [ABP1], [ABP2], [ABP2.5], [ABFP1], [ABFP2] of papers devoted to the study of the three classes $\mathbb{M}_{n}, \mathbb{I}_{n}, \mathbb{E}_{n}$. The preceding five articles contain a number of general results about these classes, and in this paper, we apply these results to the case $n=2$. Our results provide the following:

- A complete understanding of the relationship betweens the classes $\mathbb{M}_{2}$, $\mathbb{I}_{2}$ and $\mathbb{E}_{2}$.

- A classification, up to isomorphism, of the algebras in $\mathbb{M}_{2}$. 
To discuss this in more detail, note first that by Kac's realization theorem, the algebras in $\mathbb{I}_{2}$ are, up to isomorphism, the algebras of the form

$$
\mathrm{L}\left(\overline{\mathfrak{g}^{\prime}}, \sigma\right) \text {, with } \mathfrak{g} \text { affine and } \sigma \text { a finite order automorphism of } \overline{\mathfrak{g}^{\prime}} \text {. }
$$

We show in Section 10, that an algebra of this form is in $\mathbb{M}_{2}$ if and only if $\sigma$ is of first kind. (The notion of first and second kind for finite order automorphisms of $\overline{\mathfrak{g}^{\prime}}$ is recalled in $\S 7$.) In fact, our first main theorem, Theorem 10.1.1, shows that the algebras in $\mathbb{M}_{2}$ are, up to isomorphism, the algebras of the form

$$
\mathrm{L}\left(\overline{\mathfrak{g}^{\prime}}, \sigma\right) \text {, with } \mathfrak{g} \text { affine and } \sigma \text { a diagram automorphism of } \overline{\mathfrak{g}^{\prime}} ;
$$

or equivalently they are the algebras of the form

$$
\mathrm{L}\left(\overline{\mathfrak{g}^{\prime}}, \sigma\right) \text {, with } \mathfrak{g} \text { untwisted affine and } \sigma \text { a diagram automorphism of } \overline{\mathfrak{g}^{\prime}} \text {. }
$$

Using this theorem we are able in Section 10 to describe the relationship between the classes $\mathbb{M}_{2}, \mathbb{I}_{2}$ and $\mathbb{E}_{2}$ in a sequence of corollaries. This information is summarized in Figure 1.

Our second main theorem, Theorem 13.3.1, which provides a classification theorem for algebras in $\mathbb{M}_{2}$, is proved by determining when two algebras of the form (3) are isomorphic. Two key invariants that are used for this are the relative and absolute types of an algebra $\mathcal{L} \in \mathbb{M}_{2}$; these are defined to be the relative and absolute types respectively of the central closure of $\mathcal{L}$, which is finite dimensional and simple over its centroid. The absolute type of $\mathcal{L}$ is easy to compute (see 4.4.5), but the relative type requires considerably more work.

We now outline the structure of the paper, and briefly discuss some of our other results and methods. First, after some preliminaries in Section 2, we record in Sections 3, 4 and 5 the basic definitions and results we need about relative and absolute type, multiloop algebras, iterated loop algebras, and EALAs.

In Section 6, we obtain an erasing theorem, Theorem 6.2.2, for loop algebras of symmetrizable Kac-Moody Lie algebras. This theorem is an extension of a theorem in [ABP2], which allows us to replace an arbitrary $\sigma$ in (1) by an outer automorphism, just as Kac did for loop algebras of finite dimensional simple algebras in [K2, Prop. 8.5].

In Section 7, we discuss automorphisms of $\overline{\mathfrak{g}^{\prime}}$, where $\mathfrak{g}$ is affine. In particular, we show that the kind of a finite order automorphism $\sigma$ is determined by the structure of the centroid of $\mathrm{L}\left(\overline{\mathfrak{g}^{\prime}}, \sigma\right)$.

In Sections 8 and 9, we study in detail the structure of a loop algebra as in (2). Section 8 considers the rotation case when $\mathfrak{g}$ is of type $\mathrm{A}_{\ell}^{(1)}$ and $\sigma$ is a rotation of the Dynkin diagram; we see in this case that $\mathrm{L}\left(\overline{\mathfrak{g}^{\prime}}, \sigma\right)$ is a special linear algebra over a quantum torus. Section 9 considers the nontransitive case when $\sigma$ is not transitive on the Dynkin diagram; we obtain in this case a description of the relative type of $\mathrm{L}\left(\overline{\mathfrak{g}^{\prime}}, \sigma\right)$ in terms of a projection of the root system of $\mathfrak{g}$.

As indicated previously, Section 10 contains our results about the relationship between $\mathbb{M}_{2}, \mathbb{I}_{2}$ and $\mathbb{E}_{2}$.

In Sections 11 and 12, we study the isomorphism problem for loop algebras as in (2). In Section 11, we solve the problem in the rotation case, using calculations involving cyclic algebras over the field of rational functions in 2 variables. In Section 12 , we use projections of affine root systems to compute the relative type of $\mathrm{L}\left(\overline{\mathfrak{g}^{\prime}}, \sigma\right)$ in the transitive case. For this we use methods of Fuchs, Schellekens and Schweigert [FSS] and of Bausch [Bau]. 
Section 13 contains the classification theorem for algebras in $\mathbb{M}_{2}$. The algebras are listed and assigned labels in Table 2. The list consists of 14 infinite families and 9 exceptional algebras.

In the last section, Section 14, we establish links with some work of other researchers by calculating the index of each algebra $\mathcal{L}$ in $\mathbb{M}_{2}$ and the Saito extended affine root system [Sai] of each isotropic algebra $\mathcal{L}$ in $\mathbb{M}_{2}$ (see Table 4 ). Here the index of $\mathcal{L}$ is defined to be the index, in the sense of [T], of the connected component of the identity in the automorphism group of the central closure of $\mathcal{L}$ over its centroid; whereas the SEARS of $\mathcal{L}$ is defined to be the set of nonisotropic roots of a certain EALA of nullity 2 whose core modulo its centre is $\mathcal{L}$.

It turns out that by the classification theorem there is only one infinite family of algebras $\mathcal{L} \in \mathbb{M}_{2}$ that satisfy the following condition:

(AA) The absolute type of $\mathcal{L}$ is $\mathrm{A}_{k}$ for some $k \geq 1$ and the relative type of $\mathcal{L}$ is $\mathrm{A}_{r}$ for some $r \geq 0$.

(Saying that $\mathcal{L}$ has relative type $\mathrm{A}_{0}$ means that $\mathcal{L}$ is anisotropic. See Definition 3.2.1.) Moreover, we see in Sections 13 and 14 that with the exception of these algebras, an algebra in $\mathbb{M}_{2}$ is determined up to isomorphism by its relative and absolute type (together), or by its index, or by its SEARS. The second of these facts was conjectured in [GP1].

To conclude this introduction, we make a brief comment on the title of this article. Originally this project was envisaged as a sequence of three papers, beginning with [ABP1] and [ABP2], on covering algebras, an intentionally imprecise term describing the various incarnations of loop algebras that occur in the study of EALAs. (See the introduction to [ABP1].) For this reason our working title was Covering algebras III: Classification of nullity 2 multiloop Lie algebras. However, as the sequence expanded and our topic broadened, we decided that the present title more accurately reflects the content of the paper.

Acknowledgements: We wish to thank Erhard Neher and John Faulkner for many helpful conversations about multiloop algebras, extended affine Lie algebras and their root systems. In particular, John's collaboration on [ABFP1] and [ABFP2] was essential in establishing the link between these topics. We also wish to thank the Banff International Research Station, where several of the results in this paper were obtained during a Research in Teams Programme in 2005. Finally, we thank the Mathematisches Forschungsinstitut Oberwolfach for the opportunity to announce the main results of this paper in [ABPOb].

\section{Preliminaries}

In this short section, we establish some notation and conventions that we will use in this work.

We suppose throughout the paper that $\mathbb{k}$ is an algebraically closed field of characteristic 0 .

\subsection{Algebras.}

Unless indicated otherwise, algebra will mean algebra over $\mathbb{k}$.

Many of the algebras that we will consider have natural gradings, for example by $\mathbb{Z}^{n}$ or by a root lattice. Nevertheless, unless specified to the contrary, two algebras will be regarded as isomorphic if there is an ungraded isomorphism between them. 
If $F$ is a unital commutative associative $\mathbb{k}$-algebra and $\mathcal{L}_{1}$ and $\mathcal{L}_{2}$ are algebras over $F$, we write $\mathcal{L}_{1} \simeq_{F} \mathcal{L}_{2}$ to mean that $\mathcal{L}_{1}$ and $\mathcal{L}_{2}$ are isomorphic as algebras over $F$. If $F=\mathbb{k}$ we simply write $\mathcal{L}_{1} \simeq \mathcal{L}_{2}$.

If $\mathcal{L}$ is an algebra, the automorphism group of $\mathcal{L}$ over $\mathbb{k}$ is denoted by $\operatorname{Aut}_{\mathbb{k}}(\mathcal{L})$, or simply $\operatorname{Aut}(\mathcal{L})$.

If $\mathcal{L}$ is a Lie algebra, the derived algebra of $\mathcal{L}$ is (unless indicated otherwise) denoted by $\mathcal{L}^{\prime}$ and the centre of $\mathcal{L}$ is denoted by $\mathrm{Z}(\mathcal{L})$.

If $n$ is a positive integer, we let

$$
R_{n}=\mathbb{k}\left[t_{1}^{ \pm 1}, \ldots, t_{n}^{ \pm 1}\right]
$$

denote the algebra of Laurent polynomials in the variables $t_{1}, \ldots, t_{n}$ over $\mathbb{k}$.

\subsection{Matrix Lie algebras.}

Suppose that $\mathcal{A}$ is a unital associative algebra. We use the notation $\mathcal{A}^{-}$for the Lie algebra $\mathcal{A}$ under the commutator product $[x, y]=x y-y x$.

If $g \geq 1$, let $\mathrm{M}_{g}(\mathcal{A})$ denote the associative algebra of $n \times n$-matrices over $\mathcal{A}$. We then let $\mathfrak{g l}_{g}(\mathcal{A})=\mathrm{M}_{g}(\mathcal{A})^{-}$and $\mathfrak{s l}_{g}(\mathcal{A})=\mathfrak{g l}_{g}(\mathcal{A})^{\prime}$. It is well known (and easily checked) that

$$
\mathfrak{s l}_{g}(\mathcal{A})=\left\{x \in \mathfrak{g l}_{g}(\mathcal{A}) \mid \operatorname{tr}(x) \in[\mathcal{A}, \mathcal{A}]\right\} .
$$

In particular $\mathfrak{s l}_{1}(\mathcal{A})=[\mathcal{A}, \mathcal{A}]$ under the commutator product. Finally, if $u$ is a unit in $\mathrm{M}_{g}(\mathcal{A})$, we $\operatorname{define} \operatorname{Ad}(u) \in \operatorname{Aut}\left(\mathrm{M}_{g}(\mathcal{A})\right)$ by $\operatorname{Ad}(u) x=u x u^{-1}$ for $x \in \mathrm{M}_{g}(\mathcal{A})$. Then $\operatorname{Ad}(u)$ is also an automorphism of $\mathfrak{g l}_{g}(\mathcal{A})$, and we denote its restriction to $\mathfrak{s l}_{g}(\mathcal{A})$ also by $\operatorname{Ad}(u)$.

2.3. Root systems. If $\mathfrak{g}$ is a Lie algebra and $\mathfrak{h}$ is ad-diagonalizable abelian subalgebra of $\mathfrak{g}$, then we have the root space decomposition $\mathfrak{g}=\sum_{\alpha \in \mathfrak{h}^{*}} \mathfrak{g}_{\alpha}$ of $\mathfrak{g}$ with respect to $\mathfrak{h}$, where $\mathfrak{h}^{*}$ is the dual space of $\mathfrak{h}$ and $\mathfrak{g}_{\alpha}=\{x \in \mathfrak{g} \mid[h, x]=\alpha(h) x$ for $h \in \mathfrak{h}\}$. An element $\alpha \in \mathfrak{h}^{*}$ will be called a root of $\mathfrak{g}$ relative to $\mathfrak{h}$ if $\mathfrak{g}_{\alpha} \neq 0$, and the set $\left\{\alpha \in \mathfrak{h}^{*} \mid \mathfrak{g}_{\alpha} \neq 0\right\}$ is called the root system of $\mathfrak{g}$ relative to $\mathfrak{h}$. We emphasize that with this definition 0 is a root of $\mathfrak{g}$ relative to $\mathfrak{h}$ unless $\mathfrak{g}=\{0\}$.

We use the standard definition of finite (not necessarily reduced) root system, except that we regard 0 is an element of the system. Thus, in this article a finite root system is a finite subset $\Delta$ of a vector space over $\mathbb{k}$ such that $0 \in \Delta$ and $\Delta \backslash\{0\}$ is a finite root system as defined in [Bo2, Chap. VI, $\S 1$, Def. 1]. Our notation for the type of a reduced irreducible finite root system is standard and follows [Bo2, Plates I-IX]. As usual we identify $A_{1}=B_{1}=C_{1}, B_{2}=C_{2}$ and $A_{3}=D_{3}$. In addition we will use the notation $\mathrm{BC}_{k}$ for the type of the unique nonreduced irreducible finite root system of rank $k \geq 1$ [Bo2, Chap. VI, §4, no. 14].

\subsection{Symmetrizable Kac-Moody Lie algebras.}

Here we establish the notation we will use for symmetrizable Kac-Moody Lie algebras. The reader can consult [K2, Chapters 1-4], [MP, Chapter 4] or [KW, §4] for any necessary background regarding these algebras.

Suppose that $A=\left(a_{i j}\right)_{i, j \in \mathrm{I}}$ is an indecomposable symmetrizable GCM (generalized Cartan matrix), where $I$ is a finite subset of $\mathbb{Z}$. The (symmetrizable) Kac-Moody Lie algebra determined by $A$ is the Lie algebra $\mathfrak{g}=\mathfrak{g}(A)$ generated by $\mathfrak{h}$ and the symbols $\left\{e_{i}\right\}_{i \in \mathrm{I}}$ and $\left\{f_{i}\right\}_{i \in \mathrm{I}}$ subject to the relations

$$
\begin{gathered}
{[\mathfrak{h}, \mathfrak{h}]=\{0\}, \quad\left[e_{i}, f_{j}\right]=\delta_{i j} \alpha_{i}^{\vee}, \quad\left[h, e_{i}\right]=\alpha_{i}(h) e_{i}, \quad\left[h, f_{i}\right]=-\alpha_{i}(h) f_{i},} \\
\operatorname{ad}\left(e_{i}\right)^{1-a_{i j}}\left(e_{j}\right)=\operatorname{ad}\left(f_{i}\right)^{1-a_{i j}}\left(f_{j}\right)=0(i \neq j),
\end{gathered}
$$


where $\left(\mathfrak{h}, \Pi, \Pi^{\vee}\right)=\left(\mathfrak{h},\left\{\alpha_{i}\right\}_{i \in \mathrm{I}},\left\{\alpha_{i}\right\}_{i \in \mathrm{I}}^{\vee}\right)$ is a realization of $A$.

Let $\Delta$ be the set of roots (including 0) of $\mathfrak{g}$ relative to $\mathfrak{h}$, let $Q=Q(\Delta)=$ $\sum_{i \in \mathrm{I}} \mathbb{Z} \alpha_{i}$ be the root lattice of $\mathfrak{g}$, let $\mathfrak{g}=\sum_{\alpha \in Q} \mathfrak{g}_{\alpha}$ be the root space decomposition of $\mathfrak{g}$, let $W$ be the Weyl group of $\mathfrak{g}$, and let $\Delta^{\mathrm{re}}=\cup_{i \in \mathrm{I}} W \alpha_{i}$ be the set of real roots of $\mathfrak{g}$.

Let $\mathfrak{g}^{\prime}$ be the derived algebra of $\mathfrak{g}$, in which case $\mathrm{Z}(\mathfrak{g})=\mathrm{Z}\left(\mathfrak{g}^{\prime}\right)=\left\{h \in \mathfrak{h}: \alpha_{i}(h)=\right.$ 0 for $i \in \mathrm{I}$ \}. We set

$$
\overline{\mathfrak{g}^{\prime}}:=\mathfrak{g}^{\prime} / \mathrm{Z}\left(\mathfrak{g}^{\prime}\right),
$$

and let $-: \mathfrak{g}^{\prime} \rightarrow \overline{\mathfrak{g}^{\prime}}$ be the canonical map. We also let

$$
\mathfrak{h}^{\prime}:=\mathfrak{h} \cap \mathfrak{g}^{\prime}=\sum_{i \in \mathrm{I}} \mathbb{k} \alpha_{i}^{\vee} .
$$

(Of course, contrary to our convention, $\mathfrak{h}^{\prime}$ is not the derived algebra of $\mathfrak{h}$.) Note that $\mathfrak{g}^{\prime}=\sum_{\alpha \in Q}\left(\mathfrak{g}^{\prime}\right)_{\alpha}$ and $\overline{\mathfrak{g}^{\prime}}=\sum_{\alpha \in Q}\left(\overline{\mathfrak{g}^{\prime}}\right)_{\alpha}$ are $Q$-graded algebras with

for $\alpha \in Q$.

$$
\left(\mathfrak{g}^{\prime}\right)_{\alpha}=\mathfrak{g}_{\alpha} \cap \mathfrak{g} \quad \text { and } \quad\left(\overline{\mathfrak{g}^{\prime}}\right)_{\alpha}=\overline{\left(\mathfrak{g}^{\prime}\right)_{\alpha}}
$$

Our main interest is in the special cases when $A$ is of finite type or $A$ is of affine type. If $A$ is of finite type, $\mathfrak{g}$ is a split simple Lie algebra with splitting Cartan subalgebra $\mathfrak{g}$, and hence $\Delta$ is an irreducible reduced finite root system.

If $A$ is of affine type, $\mathfrak{g}$ is an affine Kac-Moody Lie algebra. We label $A$ using Tables Aff1-Aff3 of [K2, Chapter 4]. ${ }^{1}$ If $A$ has label $\mathrm{X}_{k}^{(m)}$ using this system, we say that $\mathfrak{g}$ (or $A$ ) has type $\mathrm{X}_{k}^{(m)}$. We say that $\mathfrak{g}$ (or $A$ ) is untwisted (resp. twisted) if $m=1$ (resp. $m \neq 1$ ). We will recall some more (standard) notation for affine algebras when needed in Section 7.1.

\section{Relative And ABsolute type}

In this section, we discuss some isomorphism invariants for Lie algebras that will play a crucial role in our work on classification.

\subsection{The centroid.}

We begin by recalling the definition of the centroid. Since we will work with associative algebras as well as Lie algebras later in the paper, we make the initial definitions for arbitrary (not necessarily Lie) algebras.

Definition 3.1.1. If $\mathcal{L}$ is an arbitrary algebra, the centroid of $\mathcal{L}$ is the subalgebra of $\operatorname{End}_{\mathbb{k}}(\mathcal{L})$ defined by

$$
\mathrm{C}(\mathcal{L})=\mathrm{C}_{\mathbb{k}}(\mathcal{L}):=\left\{e \in \operatorname{End}_{\mathbb{k}}(\mathcal{L}) \mid e(x y)=e(x) y=x e(y) \text { for } x, y \in \mathcal{L}\right\} .
$$

If $\mathcal{L}$ is perfect $(\mathcal{L} \mathcal{L}=\mathcal{L})$, then $\mathrm{C}(\mathcal{L})$ is commutative. Note that $\mathcal{L}$ is naturally a left $\mathrm{C}(\mathcal{L})$-module, and, if $\mathcal{L}$ is perfect, $\mathcal{L}$ is an algebra over $\mathrm{C}(\mathcal{L}) . \mathcal{L}$ is said to be fgc if $\mathcal{L}$ is finitely generated as a left $\mathrm{C}(\mathcal{L})$-module. Finally, $\mathcal{L}$ is said to be central if $\mathrm{C}(\mathcal{L})=\mathbb{k} 1$.

3.1.2. If $\mathcal{L}$ is a simple algebra and $F=\mathrm{C}(\mathcal{L})$, then $F$ is a field and $\mathcal{L}$ is central simple as an algebra over $F$. Conversely, if $F$ is an extension of $\mathbb{k}$ and $\mathcal{L}$ is a central simple algebra over $F$, then $\mathcal{L}$ is a simple algebra over $\mathbb{k}$ with centroid naturally isomorphic to $F$. (See $[J, \S \mathrm{X} .1]$ for these facts.)

\footnotetext{
${ }^{1} \mathrm{~A}$ different system is used in [MP] to label affine matrices. We will see in Remark 6.3.6 that the system we are using from [K2] is related to the absolute type of $\overline{\mathfrak{g}^{\prime}}$, whereas the system in $[\mathrm{MP}]$ is related to the relative type of $\overline{\mathfrak{g}^{\prime}}$.
} 
3.1.3. If $\mathcal{L}_{1}$ and $\mathcal{L}_{2}$ are algebras and $\sigma: \mathcal{L}_{1} \rightarrow \mathcal{L}_{2}$ is an isomorphism, then the map $\mathrm{C}(\sigma): \mathrm{C}\left(\mathcal{L}_{1}\right) \rightarrow \mathrm{C}\left(\mathcal{L}_{2}\right)$ defined by $\mathrm{C}(\sigma)(e)=\sigma e \sigma^{-1}$ is an algebra isomorphism.

\subsection{Relative and absolute type for simple fgc Lie algebras.}

Definition 3.2.1. Suppose that $\mathcal{L}$ is a simple fgc Lie algebra, and let $F=\mathrm{C}(\mathcal{L})$. Then, by 3.1.2, $\mathcal{L}$ is a finite dimensional central simple Lie algebra over $F$. Choose a $M A D F$-subalgebra $\mathcal{T}$ of $\mathcal{L}$, by which we mean a maximal ad-diagonalizable (necessarily abelian) $F$-subalgebra of $\mathcal{L}$. We say that $\mathcal{L}$ is isotropic (resp. anisotropic) if $\mathcal{T} \neq 0$ (resp. $\mathcal{T}=0)$. If $\mathcal{L}$ is isotropic, the root system of $\mathcal{L}$ relative to the adjoint action of $\mathcal{T}$ is a (possibly non-reduced) irreducible finite root system [Se2, §I.2], and the type of that root system is called the relative type of $\mathcal{L}$. If $\mathcal{L}$ is anisotropic we define the relative type of $\mathcal{L}$ to be $A_{0}$ (which is not the type of an irreducible finite root system). These notions are well-defined since $\operatorname{Aut}_{F}(\mathcal{L})$ acts transitively on the MAD $F$-subalgebras of $\mathcal{L}\left[\mathrm{Se} 2, \S \mathrm{I} .3\right.$, Thm. 2]. Also, by [J, $\S \mathrm{X} .1$, Lemma 1], $\mathcal{L} \otimes_{F} \bar{F}$ is a finite dimensional (central) simple Lie algebra over $\bar{F}$, where $\bar{F}$ is an algebraic closure of $F$. We define the absolute type of $\mathcal{L}$ to be the type of the root system of $\mathcal{L} \otimes_{F} \bar{F}$ relative to a MAD $\bar{F}$-subalgebra of $\mathcal{L} \otimes_{F} \bar{F}$. In other words, the absolute type of $\mathcal{L}$ is the relative type of $\mathcal{L} \otimes_{F} \bar{F}$.

3.2.2. We emphasize that the absolute type of a simple fgc Lie algebra is always reduced, whereas the relative type may not be. For example, $\mathcal{K}$ could have absolute type $\mathrm{A}_{2}$ and relative type $\mathrm{BC}_{1}$.

Remark 3.2.3. Suppose that $\mathfrak{g}$ is a finite dimensional simple Lie algebra. Since $\mathbb{k}$ is algebraically closed, it follows that $\mathfrak{g}$ is central [J, Lemma X.1]. So, the relative type of $\mathfrak{g}$ equals the absolute type, and as usual we call this the type of $\mathfrak{g}$.

\subsection{Relative and absolute type for prime perfect fgc Lie algebras.}

3.3.1. Recall that a Lie algebra $\mathcal{L}$ is said to be prime if $\mathcal{L} \neq 0$ and, for all ideals $\mathcal{J}$ and $\mathcal{J}$ of $\mathcal{L},[\mathcal{J}, \mathcal{J}]=0$ implies that $\mathcal{J}=0$ or $\mathcal{J}=0$.

Proposition 3.3.2. Suppose that $\mathcal{L}$ is a prime perfect fgc Lie algebra. Then $\mathrm{C}(\mathcal{L})$ is an integral domain and

$$
\widetilde{\mathcal{L}}:=\mathcal{L} \otimes_{\mathrm{C}(\mathcal{L})} \widetilde{C}(\mathcal{L})
$$

is a simple fgc Lie algebra with centroid naturally isomorphic to $\widetilde{C}(\mathcal{L})$, where $\widetilde{C}(\mathcal{L})$ denotes the quotient field of $\mathrm{C}(\mathcal{L})$. Moreover, the map $x \mapsto x \otimes 1$ identifies $\mathcal{L}$ as a $\mathrm{C}(\mathcal{L})$-subalgebra of $\widetilde{\mathcal{L}}$.

Proof. It is easy to see (and well known) that $\mathrm{C}(\mathcal{L})$ is an integral domain. It is proved in $[\mathrm{ABP} 2.5$, Prop. 8.7$]$ that $\widetilde{\mathcal{L}}$ is a finite dimensional central simple Lie algebra over $\widetilde{C}(\mathcal{L})$, so $\widetilde{\mathcal{L}}$ is simple over $\mathbb{k}$ by 3.1.2. The last statement follows from [ABP2.5, Lemma 3.3(i)].

Definition 3.3.3. Suppose that $\mathcal{L}$ is a prime perfect fgc Lie algebra. We call the Lie algebra $\widetilde{\mathcal{L}}$ in Proposition 3.3.2 the central closure of $\mathcal{L}$. We define the relative type and the absolute type of $\mathcal{L}$ to be the relative type and the absolute type respectively of the Lie algebra $\widetilde{\mathcal{L}}$. We say that $\mathcal{L}$ is isotropic (resp. anisotropic) if $\widetilde{\mathcal{L}}$ is isotropic (resp. anisotropic).

The following tells us that the notions just defined are isomorphism invariants. 
Lemma 3.3.4. Suppose that $\mathcal{L}_{1}$ and $\mathcal{L}_{2}$ are prime prefect fgc Lie algebras that are isomorphic (as $\mathbb{k}$-algebras). Then $\mathcal{L}_{1}$ and $\mathcal{L}_{2}$ have the same relative type and the same absolute type.

Proof. It is easy to check using 3.1.3 that the central closures of $\mathcal{L}_{1}$ and $\mathcal{L}_{2}$ are isomorphic. So, replacing $\mathcal{L}_{i}$ by $\widetilde{\mathcal{L}}_{i}$, we can assume that $\mathcal{L}_{i}$ is a simple fgc algebra for $i=1,2$. Let $F_{i}=\mathrm{C}\left(\mathcal{L}_{i}\right)$ and let $\bar{F}_{i}$ for a algebraic closure of $F_{i}$ for $i=1,2$. Again using 3.1.3, it is easy to see that $\mathcal{L}_{1} \otimes_{F_{1}} \bar{F}_{1}$ and $\mathcal{L}_{2} \otimes_{F_{2}} \bar{F}_{2}$ are isomorphic. Thus, it suffices to prove the statement about relative type. Suppose that $\varphi: \mathcal{L}_{1} \rightarrow \mathcal{L}_{2}$ is a $\mathbb{k}$ algebra isomorphism, and let $\gamma=\mathrm{C}(\varphi): F_{1} \rightarrow F_{2}$ be the induced isomorphism. Let $\mathcal{T}_{1}$ be a MAD $F_{1}$-subalgebra of $\mathcal{L}_{1}$, and set $\mathcal{T}_{2}=\varphi\left(\mathcal{T}_{1}\right)$. Then it is straightforward to check that $\mathcal{T}_{2}$ is a MAD $F_{2}$-subalgebra of $\mathcal{L}_{2}$, and that the map $\alpha \mapsto \gamma \circ \alpha \circ \varphi^{-1}$ is an isomorphism of the root system of $\mathcal{L}_{1}$ with respect to $\mathcal{T}_{1}$ onto the root system of $\mathcal{L}_{2}$ with respect to $\mathcal{T}_{2}$.

Proposition 3.3.5. Suppose that $\mathcal{L}$ is a prime perfect fgc Lie algebra. Then, $\mathcal{L}$ is anisotropic if and only if the only ad-nilpotent element of $\mathcal{L}$ is 0 .

Proof. Let $\widetilde{\mathcal{L}}$ be the central closure of $\mathcal{L}$. It is well known that $\widetilde{\mathcal{L}}$ is anisotropic if and only if the only ad-nilpotent element of $\widetilde{\mathcal{L}}$ is 0 . (This follows from the fact that any nonzero ad-nilpotent element of $\widetilde{\mathcal{L}}$ is part of an $\mathfrak{s l}_{2}$-triple [J, Thm. III.17].) Since the ad-nilpotent elements of $\widetilde{\mathcal{L}}$ are precisely the $\widetilde{C}(\mathcal{L})$-multiples of the ad-nilpotent elements of $\mathcal{L}$, the conclusion follows.

\section{Multiloop algebras, loop algebras and the Classes $\mathbb{M}_{n}$ AND $\mathbb{I}_{n}$}

For the rest of the paper we fix a compatible family $\left\{\zeta_{m}\right\}_{m \geq 1}$ of roots of unity in $\mathbb{k}$; this means that $\zeta_{m}$ is a primitive $m^{\text {th }}$ root of unity for $m \geq 1$ and

$$
\zeta_{m k}^{k}=\zeta_{m}
$$

for all $k, m \geq 1$. For each positive integer $m$, we let $\mathbb{Z}_{m}=\mathbb{Z} / m \mathbb{Z}=\{\bar{k} \mid k \in \mathbb{Z}\}$ be the ring of integers modulo $m$, where $\bar{k}=k+m \mathbb{Z}$ for $k \in \mathbb{Z}$.

In this section, we recall the definition of multiloop algebras and loop algebras, and discuss some of their properties. Some of these definitions and properties will be stated for arbitrary algebras.

\subsection{Multiloop algebras.}

Suppose that $n$ is a positive integer. Let

$$
S_{n}=\mathbb{k}\left[z_{1}^{ \pm 1}, \ldots, z_{n}^{ \pm 1}\right]=\sum_{\boldsymbol{k} \in \mathbb{Z}^{n}} \mathbb{k} z^{k}
$$

be the $\mathbb{Z}^{n}$-graded algebra of Laurent polynomials over $\mathbb{k}$, where $z^{k}=z_{1}^{k_{1}} \ldots z_{n}^{k_{n}}$ for $\boldsymbol{k}=\left(k_{1}, \ldots, k_{n}\right) \in \mathbb{Z}^{n}$.

Suppose that $\mathfrak{g}$ is an arbitrary algebra over $\mathbb{k}, \boldsymbol{m}=\left(m_{1}, \ldots, m_{n}\right)$ is an $n$-tuple of positive integers, $\boldsymbol{\sigma}=\left(\sigma_{1}, \ldots, \sigma_{n}\right)$ is an $n$-tuple of commuting automorphisms of $\mathfrak{g}$ such that $\boldsymbol{\sigma}^{\boldsymbol{m}}=\mathbf{1}$ (that is $\sigma^{m_{i}}=1$ for $1 \leq i \leq n$ ), and $\boldsymbol{\theta}=\left(\theta_{1}, \ldots, \theta_{n}\right.$ ) is an $n$-tuple of elements of $\mathbb{k}$ such that $\theta_{i}$ has order $m_{i}$ in $\mathbb{k}^{\times}$for $1 \leq i \leq n$.

Definition 4.1.1. (Multiloop algebra) Let $\mathbb{Z}_{m}^{n}=\mathbb{Z}_{m_{1}} \oplus \cdots \oplus \mathbb{Z}_{m_{n}}$ and let $\boldsymbol{k}=\left(k_{1}, \ldots, k_{n}\right) \mapsto \overline{\boldsymbol{k}}=\left(\bar{k}_{1}, \ldots, \bar{k}_{n}\right)$ be the canonical group homomorphism from $\mathbb{Z}^{n}$ onto $\mathbb{Z}_{\boldsymbol{m}}^{n}$. For $\boldsymbol{k}=\left(k_{1}, \ldots, k_{n}\right) \in \mathbb{Z}_{\boldsymbol{m}}^{n}$, let

$$
\mathfrak{g}^{\bar{k}}=\left\{u \in \mathfrak{g} \mid \sigma_{j} u=\theta_{j}^{k_{j}} u \text { for } 1 \leq j \leq n\right\},
$$


in which case $\mathfrak{g}=\sum_{\overline{\boldsymbol{k}} \in \mathbb{Z}_{m}^{n}} \mathfrak{g}^{\overline{\boldsymbol{k}}}$ is a $\mathbb{Z}_{\boldsymbol{m}}^{n}$-graded algebra. The multiloop algebra of $\mathfrak{g}$ relative to $\boldsymbol{\sigma}, \boldsymbol{m}$ and $\boldsymbol{\theta}$ is the $\mathbb{Z}^{n}$-graded subalgebra

$$
\mathrm{L}_{\boldsymbol{m}}(\mathfrak{g}, \boldsymbol{\sigma}, \boldsymbol{\theta})=\sum_{\boldsymbol{k} \in \mathbb{Z}^{n}} \mathfrak{g}^{\overline{\boldsymbol{k}}} \otimes z^{\boldsymbol{k}}
$$

of $\mathfrak{g} \otimes_{\mathbb{k}} S_{n}$. In particular, we set

$$
\mathrm{L}_{\boldsymbol{m}}(\mathfrak{g}, \boldsymbol{\sigma}):=\mathrm{L}_{\boldsymbol{m}}\left(\mathfrak{g}, \boldsymbol{\sigma},\left(\zeta_{m_{1}}, \ldots, \zeta_{m_{n}}\right)\right)
$$

and call $\mathrm{L}_{\boldsymbol{m}}(\mathfrak{g}, \boldsymbol{\sigma})$ the multiloop algebra of $\mathfrak{g}$ relative to $\boldsymbol{\sigma}$ and $\boldsymbol{m} .^{2}$

\section{Lemma 4.1.2.}

(a) If $\varphi \in \operatorname{Aut}(\mathfrak{g})$, then $\mathrm{L}_{\boldsymbol{m}}\left(\mathfrak{g}, \varphi \boldsymbol{\sigma} \varphi^{-1}, \boldsymbol{\theta}\right) \simeq \mathrm{L}_{\boldsymbol{m}}(\mathfrak{g}, \boldsymbol{\sigma}, \boldsymbol{\theta})$ as $\mathbb{Z}^{n}$-graded algebras, where $\varphi \boldsymbol{\sigma} \varphi^{-1}=\left(\varphi \sigma_{1} \varphi^{-1}, \ldots, \varphi \sigma_{n} \varphi^{-1}\right)$.

(b) If $r_{i}, s_{i} \in \mathbb{Z}$ are relatively prime to $m_{i}$ and $r_{i} s_{i} \equiv 1\left(\bmod m_{i}\right)$ for $1 \leq$ $i \leq n$, then $\mathrm{L}_{\boldsymbol{m}}\left(\mathfrak{g}, \boldsymbol{\sigma},\left(\theta_{1}^{r_{1}}, \ldots, \theta_{n}^{r_{n}}\right)\right)=\mathrm{L}_{\boldsymbol{m}}\left(\mathfrak{g},\left(\sigma_{1}^{s_{1}}, \ldots, \sigma_{n}^{s_{n}}\right), \boldsymbol{\theta}\right)$ as $\mathbb{Z}^{n}$-graded algebras.

(c) If $\mathbf{r}=\left(r_{1}, \ldots, r_{n}\right)$ is an $n$-tuple of integers such that $\boldsymbol{\sigma}^{\mathbf{r}}=\mathbf{1}$ and $r_{i} \mid m_{i}$ for $1 \leq i \leq n$, then $\mathrm{L}_{\mathbf{r}}\left(\mathfrak{g}, \boldsymbol{\sigma},\left(\theta_{1}^{m_{1} / r_{1}}, \ldots, \theta_{1}^{m_{n} / r_{n}}\right)\right) \simeq \mathrm{L}_{\boldsymbol{m}}(\mathfrak{g}, \boldsymbol{\sigma}, \boldsymbol{\theta})$.

Proof. (a) and (b): For the case $n=1$, see [ABP2]. The general case is easily established along similar lines.

(c): The linear extension of $x \otimes z_{1}^{k_{1}} \ldots z_{n}^{k_{n}} \mapsto x \otimes z_{1}^{\frac{m_{1}}{r_{1}} k_{1}} \ldots z_{n}^{\frac{m_{n}}{r_{n}} k_{n}}$ is an injective algebra endomorphism of $\mathfrak{g} \otimes_{\mathbb{k}} S_{n}$ which maps $\mathrm{L}_{\mathbf{r}}\left(\mathfrak{g}, \boldsymbol{\sigma},\left(\theta_{1}^{m_{1} / r_{1}}, \ldots, \theta_{1}^{m_{n} / r_{n}}\right)\right)$ onto $\mathrm{L}_{\boldsymbol{m}}(\mathfrak{g}, \boldsymbol{\sigma}, \boldsymbol{\theta})$.

4.1.3. By Lemma 4.1.2(b), any graded algebra of the form $\mathrm{L}_{\boldsymbol{m}}(\mathfrak{g}, \boldsymbol{\sigma}, \boldsymbol{\theta})$ is equal to a graded algebra of the form $\mathrm{L}_{\boldsymbol{m}}(\mathfrak{g}, \boldsymbol{\tau})$ for some $\boldsymbol{\tau}$. Thus, there is no loss of generality in considering only graded algebras of the form $\mathrm{L}_{\boldsymbol{m}}(\mathfrak{g}, \boldsymbol{\sigma})$. For the most part, we will do this in what follows.

4.1.4. By Lemma 4.1.2(c), $\mathrm{L}_{\boldsymbol{m}}(\mathfrak{g}, \boldsymbol{\sigma})$ does not depend up to (ungraded) isomorphism on the period $\boldsymbol{m}$. Therefore, when we are regarding $\mathrm{L}_{\boldsymbol{m}}(\mathfrak{g}, \boldsymbol{\sigma})$ as an ungraded algebra, we often denote it simply by $\mathrm{L}(\mathfrak{g}, \boldsymbol{\sigma})$. An algebra of the form $\mathrm{L}(\mathfrak{g}, \boldsymbol{\sigma})$ for some $\boldsymbol{\sigma}$ as above, will be called an $n$-fold multiloop algebra of $\mathfrak{g}$.

4.1.5. An $n$-fold multiloop algebra of $\mathfrak{g}$ is a Lie algebra (resp. an associative algebra) if and only if $\mathfrak{g}$ is a Lie algebra (resp. an associative algebra).

4.1.6. Using the n-tuple $\boldsymbol{m}$, we may identify $R_{n}=\mathbb{k}\left[t_{1}^{ \pm 1}, \ldots, t_{n}^{ \pm 1}\right]$ as a subalgebra of $S_{n}=\mathbb{k}\left[z_{1}^{ \pm 1}, \ldots, z_{n}^{ \pm 1}\right]$ by setting

$$
t_{i}=z_{i}^{m_{i}}
$$

for $1 \leq i \leq n$. Now $\mathfrak{g} \otimes S_{n}$ is an algebra over $S_{n}$ and hence also an algebra over $R_{n}$. Furthermore, $\mathrm{L}_{\boldsymbol{m}}(\mathfrak{g}, \boldsymbol{\sigma}, \boldsymbol{\theta})$ is an $R_{n}$-subalgebra of $\mathfrak{g} \otimes S_{n}$, and in this way $\mathrm{L}_{\boldsymbol{m}}(\mathfrak{g}, \boldsymbol{\sigma}, \boldsymbol{\theta})$ is an algebra over $R_{n}$. So we have a natural homomorphism of $R_{n}$ into the centroid of $\mathrm{L}_{\boldsymbol{m}}(\mathfrak{g}, \boldsymbol{\sigma}, \boldsymbol{\theta})$.

Since $\mathbb{k}$ is algebraically closed, finite dimensional simple algebras are central simple [J, Lemma X.1]. Thus, by [ABP2.5, Thm. 6.2 and Cor. 6.6] (see also [GP1, Lemmas 4.1.2 and 4.6.3]), we have:

\footnotetext{
${ }^{2} \mathrm{In}$ [ABP2.5], [ABFP1] and [ABFP2], the notation $\mathrm{M}_{\boldsymbol{m}}(\mathfrak{g}, \boldsymbol{\sigma})$ was used in place of $\mathrm{L}_{\boldsymbol{m}}(\mathfrak{g}, \boldsymbol{\sigma})$. We have changed notation here to be compatible with the usual notation when $n=1$. (See Definition 4.2.1 below.)
} 
Proposition 4.1.7. (The centroid of a multiloop algebra) If $\mathfrak{g}$ is finite dimensional and simple, then the natural homomorphism from $R_{n}$ into $\mathrm{C}\left(\mathrm{L}_{\boldsymbol{m}}(\mathfrak{g}, \boldsymbol{\sigma}, \boldsymbol{\theta})\right)$ is an isomorphism (which we often treat as an identification).

The following simple properties of multiloop algebras will also be useful.

Lemma 4.1.8. Suppose $\mathfrak{b}$ is an ideal of $\mathfrak{g}$ with $\sigma_{i}(\mathfrak{b})=\mathfrak{b}$ for $1 \leq i \leq n$. Let $\left.\boldsymbol{\sigma}\right|_{\mathfrak{b}}$ denote the $n$-tuple of automorphisms of $\mathfrak{b}$ obtained by restricting $\boldsymbol{\sigma}$, and let $\overline{\boldsymbol{\sigma}}$ denote the $n$-tuple of automorphisms of $\overline{\mathfrak{g}}=\mathfrak{g} / \mathfrak{b}$ induced by $\boldsymbol{\sigma}$. Then $\mathrm{L}_{\boldsymbol{m}}\left(\mathfrak{b},\left.\boldsymbol{\sigma}\right|_{\mathfrak{b}}\right)$ is a $\mathbb{Z}^{n}$-graded ideal of $\mathrm{L}_{\boldsymbol{m}}(\mathfrak{g}, \boldsymbol{\sigma})$ and $\mathrm{L}_{\boldsymbol{m}}(\mathfrak{g}, \boldsymbol{\sigma}) / \mathrm{L}_{\boldsymbol{m}}\left(\mathfrak{b},\left.\boldsymbol{\sigma}\right|_{\mathfrak{b}}\right)$ is graded isomorphic to $\mathrm{L}_{\boldsymbol{m}}(\overline{\mathfrak{g}}, \overline{\boldsymbol{\sigma}})$.

Proof. Let $-: \mathfrak{g} \rightarrow \overline{\mathfrak{g}}$ be the canonical homomorphism. Then the map $\psi: a \otimes z^{k}$ to $\bar{a} \otimes z^{\boldsymbol{k}}$ is a surjective graded homomorphism of $\mathrm{L}_{\boldsymbol{m}}(\mathfrak{g}, \boldsymbol{\sigma})$ onto $\mathrm{L}_{\boldsymbol{m}}(\overline{\mathfrak{g}}, \overline{\boldsymbol{\sigma}})$. Also $\mathrm{L}_{\boldsymbol{m}}\left(\mathfrak{b},\left.\boldsymbol{\sigma}\right|_{\mathfrak{b}}\right)$ is contained in $\operatorname{Ker}(\psi)$; and, since $\operatorname{Ker}(\psi)$ is graded, the reverse inclusion is clear.

Lemma 4.1.9. Suppose that $\mathfrak{g}$ is a Lie algebra. Then

(a) $\mathrm{L}_{\boldsymbol{m}}(\mathfrak{g}, \boldsymbol{\sigma})^{\prime}=\mathrm{L}_{\boldsymbol{m}}\left(\mathfrak{g}^{\prime},\left.\boldsymbol{\sigma}\right|_{\mathfrak{g}^{\prime}}\right)$.

(b) $\mathrm{Z}\left(\mathrm{L}_{\boldsymbol{m}}(\mathfrak{g}, \boldsymbol{\sigma})\right)=\mathrm{L}_{\boldsymbol{m}}\left(\mathrm{Z}(\mathfrak{g}),\left.\boldsymbol{\sigma}\right|_{\mathrm{Z}(\mathfrak{g})}\right)$.

(c) $\mathrm{L}_{\boldsymbol{m}}(\mathfrak{g}, \boldsymbol{\sigma})^{\prime} / \mathrm{Z}\left(\mathrm{L}_{\boldsymbol{m}}(\mathfrak{g}, \boldsymbol{\sigma})^{\prime}\right)$ is graded isomorphic to $\mathrm{L}_{\boldsymbol{m}}\left(\mathfrak{g}^{\prime} / \mathrm{Z}\left(\mathfrak{g}^{\prime}\right), \overline{\left.\boldsymbol{\sigma}\right|_{\mathfrak{g}^{\prime}}}\right)$.

Proof. (a) and (b) are clear since the left hand sides are $\mathbb{Z}^{n}$-graded. (c) now follows using (a), (b) (applied to $\mathfrak{g}^{\prime}$ ) and Lemma 4.1.8.

Finally, we recall the following result on the permanence of absolute type for multiloop algebras from [ABP2.5, Thm. 8.16].

Proposition 4.1.10. Suppose that $\mathcal{L}$ is an $n$-fold multiloop algebra of a Lie algebra $\mathfrak{g}$. If $\mathfrak{g}$ is prime, perfect and fgc, then so is $\mathcal{L}$. Moreover, in that case, the absolute type of $\mathcal{L}$ is equal to the absolute type of $\mathfrak{g}$.

4.2. Loop algebras.

The special case of Definition 4.1.1 when $n=1$ is of particular importance.

Definition 4.2.1. (Loop algebra) Suppose that $\mathfrak{g}$ is an algebra over $\mathbb{k}, m$ is a positive integer, $\sigma \in \operatorname{Aut}(\mathfrak{g})$ with $\sigma^{m}=1$, and $\theta \in \mathbb{k}^{\times}$has order $m$. The loop algebra of $\mathfrak{g}$ relative to $\sigma, m$ and $\theta$ is the $\mathbb{Z}$-graded subalgebra

$$
\mathrm{L}_{m}(\mathfrak{g}, \sigma, \theta)=\sum_{k \in \mathbb{Z}} \mathfrak{g}^{\bar{k}} \otimes z_{1}^{k}
$$

of $\mathfrak{g} \otimes S_{1}$, where $S_{1}=\mathbb{k}\left[z_{1}^{ \pm 1}\right]$ and $\mathfrak{g}^{\bar{k}}=\left\{u \in \mathfrak{g} \mid \sigma u=\theta^{k} u\right\}$ for $k \in \mathbb{Z}$. We mainly consider the special case

$$
\mathrm{L}_{m}(\mathfrak{g}, \sigma):=\mathrm{L}_{m}\left(\mathfrak{g}, \sigma, \zeta_{m}\right),
$$

which is called the loop algebra of $\mathfrak{g}$ relative to $\sigma$ and $m$. When we are regarding $\mathrm{L}_{m}(\mathfrak{g}, \sigma)$ as an ungraded algebra, we often denote it by $\mathrm{L}(\mathfrak{g}, \sigma)$. An algebra of the form $\mathrm{L}(\mathfrak{g}, \sigma)$ for some $\sigma \in \operatorname{Aut}(\mathfrak{g})$ of finite order will be called a loop algebra of $\mathfrak{g}$.

Remark 4.2.2. When $n \geq 2$, we have

$$
\mathrm{L}_{\boldsymbol{m}}(\mathfrak{g}, \boldsymbol{\sigma}, \boldsymbol{\theta}) \simeq \mathrm{L}_{m_{n}}\left(\mathrm{~L}_{\boldsymbol{m}^{\prime}}\left(\mathfrak{g}, \boldsymbol{\sigma}^{\prime}, \boldsymbol{\theta}^{\prime}\right), \sigma_{n} \otimes 1, \theta_{n}\right),
$$

where $\boldsymbol{m}^{\prime}, \boldsymbol{\sigma}^{\prime}$ and $\boldsymbol{\theta}^{\prime}$ are obtained from $\boldsymbol{m}, \boldsymbol{\sigma}$ and $\boldsymbol{\theta}$ respectively by deleting the last entry, and $\sigma_{n} \otimes 1$ denotes the restriction of $\sigma_{n} \otimes 1 \in \operatorname{Aut}\left(\mathfrak{g} \otimes S_{n-1}\right)$ to $\mathrm{L}_{\boldsymbol{m}^{\prime}}\left(\mathfrak{g}, \boldsymbol{\sigma}^{\prime}, \boldsymbol{\theta}^{\prime}\right)$ [ABP2.5, Example 5.4]. Thus multiloop algebras can be constructed by a sequence of loop constructions. 
4.3. The class $\mathbb{M}_{n}, n \geq 0$.

4.3.1. By convention, we will regard an algebra as a 0 -fold multiloop algebra of itself.

Definition 4.3.2. If $n$ is a nonnegative integer, we let $\mathbb{M}_{n}$ be the class of all algebras that are isomorphic to $n$-fold multiloop algebras of finite dimensional simple Lie algebras. Note in particular, that $\mathbb{M}_{0}$ is the class of all finite dimensional simple Lie algebras. We write $\mathcal{L} \in \mathbb{M}_{n}$ to mean that $\mathcal{L}$ is an algebra in the class $\mathbb{M}_{n}$. Algebras in $\mathbb{M}_{n}$ will be called nullity $n$ multiloop algebras.

4.4. The class $\mathbb{I}_{n}, n \geq 0$.

Definition 4.4.1. Let $n \geq 0$. We define the class of Lie algebras $\mathbb{I}_{n}$ inductively. First let $\mathbb{I}_{0}=\mathbb{M}_{0}$ be the class of finite dimensional simple Lie algebras. Second, if $n \geq 1$, we let $\mathbb{I}_{n}$ be the class of algebras isomorphic to loop algebras of algebras in $\mathbb{I}_{n-1}$. Algebras in $\mathbb{I}_{n}$ will be called nullity $n$ iterated loop algebras.

4.4.2. It follows by induction using Remark 4.2 .2 that

$$
\mathbb{M}_{n} \subseteq \mathbb{I}_{n}
$$

that is every algebra in the class $\mathbb{M}_{n}$ is in $\mathbb{I}_{n}$.

The following propositions follow from Proposition 4.1.10 and Remark 3.2.3.

Proposition 4.4.3. Any algebra $\mathcal{L}$ in $\mathbb{I}_{n}$ is a prime perfect fgc Lie algebra, and hence its relative and absolute types are defined.

Proposition 4.4.4. If $\mathcal{L}$ is isomorphic to an $n$-fold multiloop algebra of a finite dimensional simple Lie algebra $\dot{\mathfrak{g}}$, then the absolute type of $\mathcal{L}$ is equal to the type of $\dot{\mathfrak{g}}$.

4.4.5. Proposition 4.4.4 tells us how to compute the absolute type of an algebra in $\mathbb{M}_{n}$. The determination of the relative type of an algebra in $\mathbb{M}_{n}$ is a more difficult problem. In fact, in later sections, a lot of work will be devoted to the solution of this problem when $n=1$ (see Corollary 6.3.4) and most especially when $n=2$ (see Corollary 8.3.2 and Theorem 12.2.1).

\subsection{An example: the quantum torus $Q(\theta)$.}

Quantum tori, which are constructed from multiplicatively alternating matrices $\boldsymbol{q} \in M_{n}(\mathbb{k})[\mathrm{Ma}, \S 4.6 .1]$, play a fundamental role in the study of extended affine Lie algebras and multiloop algebras (see for example [BGK] and $\S 8.3$ below). In this section, we consider the special case that will be important for our purposes: the case when $n=2$

4.5.1. If $\theta \in \mathbb{k}^{\times}$, let $Q(\theta)$ be the unital associative algebra presented by the generators $x_{1}, x_{2}, x_{1}^{-1}, x_{2}^{-1}$ subject to the inverse relations $x_{1} x_{1}^{-1}=x_{1}^{-1} x_{1}=1$, $x_{2} x_{2}^{-1}=x_{2}^{-1} x_{2}=1$, and the relation

$$
x_{1} x_{2}=\theta x_{2} x_{1} .
$$

$Q(\theta)$ has a $\mathbb{Z}^{2}$-grading $Q(\theta)=\bigoplus_{\boldsymbol{k} \in \mathbb{Z}^{2}} Q(\theta)^{\boldsymbol{k}}$ with $Q(\theta)^{\left(k_{1}, k_{2}\right)}=\mathbb{k} x_{1}^{k_{1}} x_{2}^{k_{2}}$. We call $Q(\theta)$ the quantum torus determined by $\theta$, and we call $x_{1}, x_{2}, x_{1}^{-1}, x_{2}^{-1}$ the distinguished generators of $Q(\theta)$ over $\mathbb{k}$.

4.5.2. For the rest of the section, assume that $\theta$ is an element of $\mathbb{k}^{\times}$of finite order $m$. 
4.5.3. It is easy to check that the centre $Z(Q(\theta))$ of $Q(\theta)$ is the algebra generated by $x_{1}^{ \pm m}$ and $x_{2}^{ \pm m}$. Thus we can identify

$$
R_{2}=\mathbb{k}\left[t_{1}^{ \pm 1}, t_{2}^{ \pm 2}\right]=Z(Q(\theta))
$$

by setting

$$
t_{1}=x_{1}^{m} \quad \text { and } \quad t_{2}=x_{2}^{m} .
$$

In particular, $Q(\theta)$ is an algebra over $R_{2}$. Moreover, $Q(\theta)$ is a free $R_{2}$-module with basis $\left\{x_{1}^{k_{1}} x_{2}^{k_{2}} \mid 0 \leq k_{1}, k_{2} \leq m-1\right\}$.

To describe $Q(\theta)$ as a multiloop algebra, we need some notation (which will also be used later in Section 8).

4.5.4. (Pauli matrices) If $F$ is a commutative associative unital $\mathbb{k}$-algebra, $u$ is a unit in $F$, and $m \geq 1$, we let

$$
d_{m}(u)=\operatorname{diag}\left(1, u, u^{2}, \ldots, u^{m-1}\right) \quad \text { and } \quad p_{m}(u)=\left[\begin{array}{cccc}
0 & \ldots & 0 & u \\
1 & \ddots & & 0 \\
\vdots & \ddots & \ddots & \vdots \\
0 & \ldots & 1 & 0
\end{array}\right]
$$

in $\mathrm{M}_{m}(F)$. (If $m=1$, we interpret $d_{1}(u)=[1]$ and $p_{1}(u)=[u]$.) Now $d_{m}(u)$ and $p_{m}(u)$ are units in $\mathrm{M}_{m}(F)$, so we can set

$$
D_{m}(u)=\operatorname{Ad}\left(d_{m}(u)\right) \quad \text { and } \quad P_{m}(u)=\operatorname{Ad}\left(p_{m}(u)\right)
$$

in the automorphism group of $\mathrm{M}_{m}(F), \mathfrak{g l}_{m}(F)$ or $\mathfrak{s l}_{m}(F)$. (See Section 2.2 for the notation Ad.) Note that $p_{m}(u)^{m}=u I$, so $P_{m}(u)^{m}=I$. Also, if $u^{m}=1$, then $d_{m}(u)^{m}=1$ and $D_{m}(u)^{m}=1$.

\subsubsection{Now let}

$$
\boldsymbol{m}=(m, m) \text { and } \boldsymbol{\theta}=(\theta, \theta) .
$$

We have seen in 4.1.6 that for any algebra $\mathfrak{g}$ and any pair $\boldsymbol{\sigma}=\left(\sigma_{1}, \sigma_{2}\right)$ of period $m$ automorphisms of $\mathfrak{g}$, the multiloop algebra $\mathrm{L}_{\boldsymbol{m}}(\mathfrak{g}, \boldsymbol{\sigma}, \boldsymbol{\theta})$ is naturally an $R_{2}$-algebra, where $R_{2}=\mathbb{k}\left[t_{1}^{ \pm 1}, t_{2}^{ \pm 1}\right]$ with $t_{1}=z_{1}^{m}$ and $t_{2}=z_{2}^{m}$. With this in mind we have:

Lemma 4.5.6. Let $\boldsymbol{\sigma}=\left(\sigma_{1}, \sigma_{2}\right)$, where

$$
\sigma_{1}=D_{m}(\theta) \quad \text { and } \quad \sigma_{2}=P_{m}(1)
$$

in Aut $\left(\mathrm{M}_{m}(\mathbb{k})\right)$. Let $g$ be a positive integer and identify $\mathrm{M}_{g m}(\mathbb{k})=\mathrm{M}_{g}\left(\mathrm{M}_{m}(\mathbb{k})\right)$ in the usual fashion. With this identification, let $\tilde{\boldsymbol{\sigma}}=\left(\tilde{\sigma}_{1}, \tilde{\sigma}_{2}\right)$ be the pair of automorphisms of $\mathrm{M}_{g m}(\mathbb{k})$ induced by $\boldsymbol{\sigma}$ acting on the $m \times m$-blocks. Then

(a) $Q(\theta) \simeq_{R_{2}} \mathrm{~L}_{\boldsymbol{m}}\left(\mathrm{M}_{m}(\mathbb{k}), \boldsymbol{\sigma}, \boldsymbol{\theta}\right)$.

(b) $\mathrm{M}_{g}(Q(\theta)) \simeq_{R_{2}} \mathrm{~L}_{\boldsymbol{m}}\left(\mathrm{M}_{g m}(\mathbb{k}), \tilde{\boldsymbol{\sigma}}, \boldsymbol{\theta}\right)$.

(c) $\mathfrak{g l}_{g}(Q(\theta)) \simeq_{R_{2}} \mathrm{~L}_{\boldsymbol{m}}\left(\mathfrak{g l}_{g m}(\mathbb{k}), \tilde{\boldsymbol{\sigma}}, \boldsymbol{\theta}\right)$ and under this isomorphism

$$
\mathfrak{s l}_{g}(Q(\theta)) \simeq_{R_{2}} \mathrm{~L}_{\boldsymbol{m}}\left(\mathfrak{s l}_{g m}(\mathbb{k}),\left.\tilde{\boldsymbol{\sigma}}\right|_{\mathfrak{s l}_{g m}(\mathbb{k})}, \boldsymbol{\theta}\right) .
$$

(d) $\mathfrak{g l}_{g}(Q(\theta))=R_{2} 1 \oplus \mathfrak{s l}_{g}(Q(\theta))$ as Lie algebras over $R_{2}$.

(e) If $g m>1$, the natural homomorphism $R_{2} \mapsto C\left(\mathfrak{s l}_{g}(Q(\theta))\right)$ is an isomorphism. 
Proof. (a): Let $p=p_{m}(1)$ and $d=d_{m}(\theta)^{-1}$ in $\mathrm{M}_{m}(\mathbb{k})$. Then one checks that $p d=\theta d p$,

$$
\sigma_{1}(p)=\theta p, \quad \sigma_{2}(p)=p, \quad \sigma_{1}(d)=d \quad \text { and } \quad \sigma_{2}(d)=\theta d .
$$

So $\tilde{x}_{1}:=p \otimes z_{1}$ and $\tilde{x}_{2}:=d \otimes z_{2}$ are units in $\mathrm{L}_{\boldsymbol{m}}\left(\mathrm{M}_{m}(\mathbb{k}), \boldsymbol{\sigma}, \boldsymbol{\theta}\right)$ satisfying $\tilde{x}_{1} \tilde{x}_{2}=$ $\theta \tilde{x}_{2} \tilde{x}_{1}$. Thus we have an algebra homomorphism $\varphi: Q(\theta) \rightarrow \mathrm{L}_{\boldsymbol{m}}\left(\mathrm{M}_{m}(\mathbb{k}), \boldsymbol{\sigma}, \boldsymbol{\theta}\right)$ such that $x_{i} \mapsto \tilde{x}_{i}$ for $i=1,2$. Using the well known fact that $\left\{p^{k_{1}} d^{k_{2}} \mid 0 \leq k_{1}, k_{2} \leq\right.$ $m-1\}$ is a basis for $\mathrm{M}_{m}(\mathbb{k})$, it is easy to check that $\varphi$ is an isomorphism (see the argument in [ABP2.5, Example 9.8]). Moreover, under $\varphi$ we have $x_{1}^{m} \mapsto \tilde{x}_{1}^{m}=$ $p^{m} \otimes z_{1}^{m}=1 \otimes t_{1}$ and $x_{2}^{m} \mapsto \tilde{x}_{2}^{m}=d^{m} \otimes z_{2}^{m}=1 \otimes t_{2}$, so $\varphi$ is $R_{2}$-linear.

(b): This follows from (a).

(c): The first isomorphism in (c) follows from (b); and then (5) follows from Lemma 4.1.9(a).

(d): Now $\mathfrak{g l}_{g m}(\mathbb{k})=\mathbb{k} 1 \oplus \mathfrak{s l}_{g m}(\mathbb{k})$. So we have $\mathrm{L}_{\boldsymbol{m}}\left(\mathfrak{g l}_{g m}(\mathbb{k}), \tilde{\boldsymbol{\sigma}}, \boldsymbol{\theta}\right)=R_{2} 1 \oplus$ $\mathrm{L}_{\boldsymbol{m}}\left(\mathfrak{s l}_{g m}(\mathbb{k}),\left.\tilde{\boldsymbol{\sigma}}\right|_{\mathfrak{s l}_{g m}(\mathbb{k})}, \boldsymbol{\theta}\right)$, and (d) follows using (c).

(e): This follows from (5) using Proposition 4.1.7.

Remark 4.5.7. Parts (d) and (e) of the lemma can be proved without using multiloop algebras. In particular, if $g>1$, the Lie algebra $\mathfrak{s l}_{g}(Q(\theta))$ is graded by the root system $\mathrm{A}_{g-1}$, and hence (e) follows from [BN, Thm. 5.15].

Proposition 4.5.8. Suppose $g$ is a positive integer and $\theta \in \mathbb{k}^{\times}$has order $m$.

(a) If $g m>1$, then $\mathfrak{s l}_{g}(Q(\theta)) \in \mathbb{M}_{2}$.

(b) If $m>1$, then $\mathfrak{s l}_{1}(Q(\theta))$ has relative type $A_{0}$; that is, $\mathfrak{s l}_{1}(Q(\theta))$ is anisotropic.

Proof. (a) follows from (5) since $\mathfrak{s l}_{g m}(\mathbb{k})$ is simple. For (b), let $\mathcal{L}=\mathfrak{s l}_{1}(Q(\theta)$ ), and suppose for contradiction (see Lemma 3.3.5) that $\mathcal{L}$ contains a nonzero ad-nilpotent element $x$. Now $\mathcal{L}$ is a $\mathbb{Z}^{2}$-graded subalgebra of $Q(\theta)^{-}$, so the homogeneous components of $x$ lie in $\mathcal{L}$. Replacing $x$ by its component of highest degree (using the lexicographic order), we can assume that $x$ is homogeneous. But since $\mathcal{L} \in \mathbb{M}_{2}$ is prime by Proposition 4.4.3, $\mathcal{L}$ has trivial centre. Hence, $[x, y] \neq 0$ for some nonzero homogeneous $y \in \mathcal{L}$. Since the homogeneous components of $Q(\theta)$ are each spanned by a unit, it follows that $[x, y]=a x y$ for some $a \in \mathbb{k}^{\times}$. But then, by induction, we have $\operatorname{ad}(x)^{r} y=a^{r} x^{r} y$ for $r \geq 1$, giving a contradiction.

4.5.9. Proposition 4.5 .8 holds more generally for the quantum torus $\mathbb{k}_{q}[\mathrm{Ma}$, \$4.6.1] constructed from a multiplicatively alternating matrix $\boldsymbol{q} \in M_{n}(\mathbb{k})$ such that each $q_{i j}$ has finite order in $\mathbb{k}^{\times}$. Specifically, if either $g>1$ or some $q_{i j} \neq 1$, then $\mathfrak{s l}_{g}\left(\mathbb{k}_{\boldsymbol{q}}\right) \in \mathbb{M}_{n}$. Moreover, $\mathfrak{s l}_{1}\left(\mathbb{k}_{\boldsymbol{q}}\right)$ is anisotropic if some $q_{i j} \neq 1$. Our proof of these statements uses [ABFP1, Thm. 9.2.1] as well as the argument above. We omit the details since we will not use these facts.

\section{Extended AfFine Lie algebras and the Class $\mathbb{E}_{n}$}

In this section, we recall some background on extended affine Lie algebras.

\subsection{The definition.}

Following [Neh2] and [Neh3, §6.11], we have the following:

Definition 5.1.1. (EALA) Let $\mathfrak{g}$ be a Lie algebra satisfying the following axioms: (EA1) $\mathfrak{g}$ has a nondegenerate invariant symmetric bilinear form $(\mid)$. 
(EA2) $\mathfrak{g}$ contains a nontrivial finite dimensional self-centralizing ad-diagonalizable subalgebra $\mathfrak{h}$.

Let $\mathfrak{g}=\sum_{\alpha \in \mathfrak{h}^{*}} \mathfrak{g}_{\alpha}$ be the root space decomposition of $\mathfrak{g}$ with respect to $\mathfrak{h}$, and let

$$
\Phi:=\left\{\alpha \in \mathfrak{h}^{*}: \mathfrak{g}_{\alpha} \neq 0\right\}
$$

be the root system of $\mathfrak{g}$ relative to $\mathfrak{h}$. The form $(\mid)$ restricted to $\mathfrak{h}$ is nondegenerate, and hence, as usual, we can transfer $(\mid)$ to a nondegenerate symmetric bilinear form on the dual space $\mathfrak{h}^{*}$ of $\mathfrak{h}$. Let

$$
\Phi^{\times}=\{\alpha \in \Phi \mid(\alpha \mid \alpha) \neq 0\} \quad \text { and } \quad \Phi^{0}=\{\alpha \in \Phi \mid(\alpha \mid \alpha)=0\}
$$

be the sets of nonisotropic (resp. isotropic) roots in $\Phi$. Let

$$
\mathfrak{g}_{c}:=\text { subalgebra of } \mathfrak{g} \text { generated by } \mathfrak{g}_{\alpha}, \alpha \in \Phi^{\times}
$$

be the core of $\mathfrak{g}$. We say that $(\mathfrak{g},(\mid), \mathfrak{h})$ is an extended affine Lie algebra (EALA) if in addition to (EA1) and (EA2), the following axioms hold:

(EA3) $\operatorname{ad}(x)$ is locally nilpotent for $x \in \mathfrak{g}_{\alpha}, \alpha \in \Phi^{\times}$.

(EA4) $\Phi^{\times}$cannot be decomposed as the union of two orthogonal nonempty subsets.

(EA5) The centralizer of $\mathfrak{g}_{c}$ in $\mathfrak{g}$ is contained in $\mathfrak{g}_{c}$.

(EA6) The subgroup $\left\langle\Phi^{0}\right\rangle$ of $\mathfrak{h}^{*}$ generated by $\Phi^{0}$ is finitely generated.

We will then often say for short that $\mathfrak{g}$ is an EALA.

5.1.2. An EALA over $\mathbb{C}$ such that $\Phi$ is a discrete subgroup of $\mathfrak{h}^{*}$ is called a discrete $E A L A$ [Neh3, 6.14]. (In fact, (EA6) is redundant in this definition [Neh3, 6.15].) Discrete EALAs have been studied in a number of papers including [H-KT], [BGK], $[\mathrm{AABGP}],[\mathrm{ABGP}],[\mathrm{AG}]$ and $[\mathrm{ABP} 1]$ where, beginning with $[\mathrm{AABGP}]$, they were called tame EALAs.

5.1.3. The properties of the root systems of discrete EALAs were developed in [AABGP, Chapter 2]. It was shown that these root systems can be described using a finite root system together with a family of semilattices.

5.1.4. More generally, as described in $[\mathrm{Neh} 3, \S 6]$, if $\Phi$ is the root system of an EALA $\mathfrak{g}$ then $\Phi$ has the following properties: $\Phi$ is a reduced symmetric affine reflection system with irreducible finite quotient root system; all root strings are unbroken; $\left\langle\Phi^{0}\right\rangle$ is finitely generated; and $\Phi^{0} \subseteq \Phi^{\times}+\Phi^{\times}$. (The interested reader can consult [Neh3] or [LN] for the terms used here. We will not use them subsequently.) As a consequence, it follows from [LN, §4-5] (see also [Neh3, §3.7]) that the structure of $\Phi$ can be described in terms of extension data for the finite quotient root system of $\Phi$. This generalizes the approach mentioned in 5.1.3 that uses semilattices. As a result, most of the basic properties of discrete EALAs carry over to general EALAs with similar and sometimes shorter proofs. In a few cases below we will only sketch an argument along these lines, as the details are not hard to fill in.

\subsection{5. (The quotient type of an EALA)}

Suppose that $(\mathfrak{g},(\mid), \mathfrak{h})$ is an EALA with root system $\Phi$. Then, using the properties mentioned in $\mathbf{5 . 1 . 4}$, it is easy to deduce the following facts about $\Phi$ from standard facts about finite root systems. First, let

$$
V=\operatorname{span}_{\mathbb{Q}}(\Phi)
$$


in $\mathfrak{h}^{*}$. Then $V$ is finite dimensional over $\mathbb{Q}$. Further, we can and do normalize $(\mid)$, by replacing it by a $\mathbb{k}^{\times}$multiple of itself, so that $(\alpha \mid \beta) \in \mathbb{Q}$ and $(\alpha \mid \alpha) \geq 0$ for $\alpha, \beta \in \Phi$. In that case, $(\mid)$ restricts to a $\mathbb{Q}$-bilinear form $V \times V \rightarrow \mathbb{Q}$; and this restriction, which we also denote by $(\mid)$, is positive semi-definite (that is $(\alpha \mid \alpha) \geq 0$ for $\alpha \in V$ ). We let

$$
V^{0}=\operatorname{rad}(V)
$$

be the radical of this form, and we set

$$
\bar{V}=V / V^{0}
$$

with canonical map $-: V \rightarrow \bar{V}$. So $(\mid)$ induces a positive definite $\mathbb{Q}$-bilinear form on $\bar{V}$, which we again denote by $(\mid)$. Further, $\bar{\Phi}$ is an irreducible (possibly nonreduced) finite root system in $\bar{V}$. We call $\bar{\Phi}$ the finite quotient root system for $(\mathfrak{g},(\mid), \mathfrak{h})$, or simply for $\mathfrak{g}$, and we call its type the quotient type of $\mathfrak{g}$.

Remark 5.1.6. Suppose that $\mathfrak{g}$ is a discrete EALA (over $\mathbb{C}$ ). Then the properties described in 5.1.5 hold with the rational field $\mathbb{Q}$ everywhere replaced by the real field $\mathbb{R}$, in which case one obtains a finite quotient root system over $\mathbb{R}$ rather than $\mathbb{Q}[$ AABGP, Chap. 1]. It is not difficult to check that this finite root system over $\mathbb{R}$ is obtained by base field extension (as described in [Bo2, Chap. VI, §1, Remark 1]) from the one over $\mathbb{Q}$. So these two finite root systems have the same type. Hence, the notion of quotient type defined above coincides with the notion of type defined in [AABGP, p. 27].

Lemma 5.1.7. Suppose that $\mathfrak{g}$ is an EALA with root system $\Phi$. Then

(a) $\mathfrak{g}_{c}$ is a perfect ideal of $\mathfrak{g}$.

(b) $\Phi^{0}=\{\alpha \in \Phi \mid \mathbb{Z} \alpha \subseteq \Phi\}$. Hence, $\Phi^{0}$ and $\Phi^{\times}$are determined by $\Phi$ (without reference to the form $(\mid))$.

Proof. In the case of discrete EALAs, (a) is proved in [AG, §1], and (b) follows from [AABGP, Cor. 2.31]. The arguments in general follow the same lines, as discussed in 5.1.4.

\subsection{The nullity of an EALA.}

Definition 5.2.1. Suppose that $\mathfrak{g}$ is an EALA with root system $\Phi$. Since the additive group of $\mathfrak{h}^{*}$ is torsion free, it follows from (EA6) that $\left\langle\Phi^{0}\right\rangle$ is a finitely generated free abelian group. We define the nullity of $\mathfrak{g}$ to be the rank of the group $\left\langle\Phi^{0}\right\rangle$.

Remark 5.2.2. Let $\mathfrak{g}$ be an EALA with notation as in 5.1.5.

(a) Using extension data, one can easily see that $\operatorname{span}_{\mathbb{Q}}\left(\Phi^{0}\right)=V^{0}$ (see [AABGP, $(2.10)$ and $(2.11)(b)]$ in the discrete case). Thus, the nullity of $\mathfrak{g}$ equals $\operatorname{dim}_{\mathbb{Q}}\left(V^{0}\right)$.

(b) Suppose that $\mathfrak{g}$ is discrete (over $\mathbb{C}$ ). Let $V_{\mathbb{R}}$ be the real span of $R$ and let $V_{\mathbb{R}}^{0}$ be the radical of the restriction of $(\mid)$ to $V_{\mathbb{R}}$. Then, by [AABGP, Prop. 1.4 and Cor. 2.31], the rank of $\left\langle\Phi^{0}\right\rangle$ equals $\operatorname{dim}_{\mathbb{R}}\left(V_{\mathbb{R}}^{0}\right)$. Thus, the notion of nullity defined above coincides with the notion of nullity defined in [AABGP, p. 27].

Using results of $[\mathrm{ABFP} 2]$ and $[\mathrm{ABGP}]$, we have the following characterizations of EALAs of nullity 0 and 1.

Proposition 5.2.3. 
(a) If $\mathfrak{g}$ is a finite dimensional simple Lie algebra with Killing form $(\mid)$ and Cartan subalgebra $\mathfrak{h}$, then $(\mathfrak{g},(\mid), \mathfrak{h})$ is an EALA of nullity 0. Conversely, if $(\mathfrak{g},(\mid), \mathfrak{h})$ is an EALA of nullity 0, then $\mathfrak{g}$ is a finite dimensional simple Lie algebra and $\mathfrak{g}_{c}=\mathfrak{g}$.

(b) If $\mathfrak{g}$ is an affine Kac-Moody Lie algebra with normalized invariant form ( | ) (see $[\mathrm{K} 2, \S 6.2]$ or $\mathbf{7 . 1 . 1}$ below) and with distinguished Cartan subalgebra $\mathfrak{h}$, then $(\mathfrak{g},(\mid), \mathfrak{h})$ is an EALA of nullity 1. Conversely, if $(\mathfrak{g},(\mid), \mathfrak{h})$ is an EALA of nullity 1, then $\mathfrak{g}$ is isomorphic to an affine Kac-Moody Lie algebra and $\mathfrak{g}_{c}=\mathfrak{g}^{\prime}$.

Proof. (a): The first statement follows from standard facts about finite dimensional simple Lie algebras. For the converse, suppose that $(\mathfrak{g},(\mid), \mathfrak{h})$ is an EALA of nullity 0 with root system $\Phi$. Then, by [Neh3, Prop 6.4], $\mathfrak{g}_{c}$ is a Lie $(\Lambda, \bar{\Phi})$-torus, where $\Lambda=\left\langle\Phi^{0}\right\rangle=\{0\}$ (see 5.3.3 for this terminology). So, by [ABFP2, Remark 1.2.4], $\mathfrak{g}_{c}$ is finite dimensional and simple. Now let $d \in \mathfrak{g}$. Then, since $\mathfrak{g}_{c}$ is an ideal of $\mathfrak{g}$ and every derivation of $\mathfrak{g}_{c}$ is inner, it follows that $d-e$ centralizes $\mathfrak{g}_{c}$ for some $e \in \mathfrak{g}_{c}$. So by (EA5), $d=e$. Hence, $\mathfrak{g}=\mathfrak{g}_{c}$ is finite dimensional simple.

(b): The first statement follows from standard facts about affine algebras. The second statement is proved for discrete EALAs over $\mathbb{C}$ in $[\mathrm{ABGP}]$, and the proof given there can be easily adapted to handle the general case (as discussed in 5.1.4). Indeed, suppose that $(\mathfrak{g},(\mid), \mathfrak{h})$ is an EALA of nullity 1 . Then, using extension data, one sees, as in [ABGP, p. 677-678], that the root system $\Phi$ of $\mathfrak{g}$ is one of the root systems for an affine algebra. Using that information the proof of Theorem 2.31 of $[\mathrm{ABGP}]$ gives our conclusion.

5.2.4. If $\mathfrak{g}$ is a finite dimensional simple (resp. an affine Kac-Moody) Lie algebra, we will subsequently regard $\mathfrak{g}$ as an EALA with the choices of $(\mid)$ and $\mathfrak{h}$ made in the first sentence of Proposition 5.2.3(a) (resp. Proposition 5.2.3(b)).

5.2.5. If $\mathfrak{g}$ is finite dimensional simple, the quotient type of $\mathfrak{g}$ is the type of $\mathfrak{g}$. Also, for each affine GCM $A$, the quotient type of $\mathfrak{g}(A)$ is well known (see Remark 6.3.5 below).

\subsection{Centreless cores of EALAs and Lie tori.}

Definition 5.3.1. Suppose that $\mathfrak{g}$ is an EALA with root system $\Phi$. We let

$$
\mathfrak{g}_{c c}=\mathfrak{g}_{c} / Z\left(\mathfrak{g}_{c}\right) .
$$

Since $\mathfrak{g}_{c}$ is perfect, $\mathfrak{g}_{c c}$ is centreless (that is it has trivial centre). For this reason $\mathfrak{g}_{c c}$ is called the centreless core of $\mathfrak{g}$.

Example 5.3.2. If $\mathfrak{g}$ is a finite dimensional simple Lie algebra (resp. an affine Kac-Moody Lie algebra) then by Proposition 5.2.3 we have $\mathfrak{g}_{c c} \simeq \mathfrak{g}$ (resp. $\left.\mathfrak{g}_{c c}=\overline{\mathfrak{g}^{\prime}}\right)$.

5.3.3. (Lie tori) In order to give an axiomatic description of the centreless cores of EALAs, Yoshii introduced Lie tori in [Y2]. To describe these, let $\Lambda$ be a finitely generated free abelian group and let $\Delta$ be an irreducible finite root system with root lattice $Q(\Delta)$,

A Lie $(\Lambda, \Delta)$-torus is a Lie algebra $\mathcal{L}$ with compatible gradings by $\Lambda$ and $Q(\Delta)$ so that the $Q(\Delta)$-support of $\mathcal{L}$ is contained in $\Delta$ and four natural axioms hold. We will not need to refer directly to these axioms and instead direct the interested reader to [Neh1], [ABFP2, §1] or (for an equivalent definition) [Neh3, §5.1]. 
If $\mathcal{L}$ is a Lie $(\Lambda, \Delta)$-torus, we say that $\mathcal{L}$ has full root support if the $Q(\Delta)$-support of $\mathcal{L}$ equals $\Delta$. There is no loss of generality in assuming this when convenient, since it always holds if we replace $\Delta$ by a suitable sub-root-system of $\Delta$. (See [ABFP2, Remark 1.1.11] for more about this.)

If $\mathcal{L}$ is a centreless Lie $(\Lambda, \Delta)$-torus, we say that $\mathcal{L}$ is invariant if there exists a nondegenerate invariant graded symmetric bilinear form on $\mathcal{L}$. (This is equivalent to the definition in [Neh3, §5.1] in view of [ABFP2, Prop. 1.2.2(vi)].)

We have the following relationship between EALAs and centreless Lie tori. (See [Neh2] or [Neh3, §6]. For discrete EALAs, see [AG, §1] and [Y2].)

Proposition 5.3.4. Let $\Lambda$ be a free abelian group of rank $n$, let $\Delta$ be an irreducible finite root system of type $X_{k}$. Then, a Lie algebra $\mathcal{L}$ is isomorphic to the centreless core of an EALA of nullity $n$ and quotient type $X_{k}$ if and only if $\mathcal{L}$ is isomorphic to an invariant centreless Lie $(\Lambda, \Delta)$-torus with full root support.

Remark 5.3.5. Suppose that $\mathbb{k}=\mathbb{C}$. In [Y2], Yoshii showed, using some classification results for centreless Lie tori, that every centreless Lie torus over $\mathbb{C}$ is invariant. Also, it follows from [Y2] that the term "EALA" can be replaced by the term "discrete EALA" in Proposition 5.3.4. Hence, centreless cores of EALAs are the same algebras as centerless cores of discrete EALAs. We will not make use of these facts in this article.

5.3.6. Centreless Lie tori and hence centreless cores of EALAs have been characterized as certain "matrix algebras" over (in general) infinite dimensional graded coordinate algebras using theorems that are called coordinatization theorems. There is one such theorem for each quotient type; the reader is referred to [AF] for a overview of this work by many authors. This approach provides a wealth of detailed information about centreless cores, but we will not use it in this work, except to calculate some indices in Section 14.2.

\subsection{The class $\mathbb{E}_{n}$.}

Definition 5.4.1. If $n$ is a nonnegative integer, let $\mathbb{E}_{n}$ be the class of all Lie algebras that are isomorphic to the centreless core of an EALA of nullity $n$. We call algebras in $\mathbb{E}_{n}$ nullity $n$ centreless cores.

5.4.2. Note that Proposition 5.3.4 gives a characterization of the algebras in $\mathbb{E}_{n}$ in terms of centreless Lie tori.

For each $n \geq 0$, we have now defined three classes $\mathbb{M}_{n}, \mathbb{I}_{n}$ and $\mathbb{E}_{n}$ of Lie algebras. For $n=0$, we have the following:

Proposition 5.4.3. $\mathbb{M}_{0}=\mathbb{I}_{0}=\mathbb{E}_{0}$ is the class of finite dimensional simple Lie algebras.

Proof. All that must be proved is that $\mathbb{E}_{0}$ is the class of finite dimensional simple Lie algebras, and this follows from Proposition 5.2.3(a).

\subsection{Fgc algebras in $\mathbb{E}_{n}$.}

We have the following from $[\mathrm{ABFP} 2]$ and $[\mathrm{A}]$.

Theorem 5.5.1. Suppose that $\mathcal{L}$ is an fgc centreless Lie $(\Lambda, \Delta)$-torus with full root support, where $\Lambda$ has rank $n$ and $\Delta$ has type $X_{k}$. Then, $\mathcal{L} \in \mathbb{M}_{n}$ and the relative type (as defined in Definition 3.3.3) of $\mathcal{L}$ is $X_{k}$. 
Proof. The fact that $\mathcal{L} \in \mathbb{M}_{n}$ is proved in Theorem 3.3.1 of [ABFP2], and the fact that $\mathcal{L}$ has relative type $X_{k}$ is proved in [A].

\section{Corollary 5.5.2.}

(a) If $\mathfrak{g}$ is an EALA of nullity $n$ such that $\mathfrak{g}_{c c}$ is fgc, then $\mathfrak{g}_{c c} \in \mathbb{M}_{n}$, $\mathfrak{g}_{c c}$ is isotropic, and the relative type of $\mathfrak{g}_{c c}$ is the quotient type of $\mathfrak{g}$.

(b) $\mathbb{M}_{n} \cap \mathbb{E}_{n}$ is the class of fgc algebras in $\mathbb{E}_{n}$.

Proof. (a) Let $X_{k}$ be the quotient type of $\mathfrak{g}$ and let $\mathcal{L}=\mathfrak{g}_{c c}$. By Proposition 5.3.4 and Theorem 5.5.1, we know that $\mathcal{L} \in \mathbb{M}_{n}$ and that the relative type of $\mathcal{L}$ is $X_{k}$. Finally, since $k>0$, it follows that $\mathcal{L}$ is isotropic.

(b) follows from (a) and Proposition 4.4.3.

\section{Loop algebras of Symmetrizable KaC-Moody Lie Algebras}

To obtain our main results, we will need to understand loop algebras of finite dimensional simple Lie algebras and loop algebras of affine Lie algebras. We consider in this section the more general topic of loop algebras of symmetrizable Kac-Moody Lie algebras.

We assume throughout the section that $\mathfrak{g}=\mathfrak{g}(A)$ is the Kac-Moody Lie algebra determined by an indecomposable symmetrizable $G C M A=\left(a_{i j}\right)_{i, j \in \mathrm{I}}$. We use the notation of Section 2.4.

\subsection{Automorphisms of symmetrizable Kac-Moody Lie algebras.}

We now recall some facts about automorphisms of $\mathfrak{g}, \mathfrak{g}^{\prime}$ and $\overline{\mathfrak{g}^{\prime}}$.

6.1.1. We begin by discussing some subgroups of $\operatorname{Aut}(\mathfrak{g})$.

First let $\operatorname{Aut}(A)$ be the group of automorphisms of the GCM $A$; that is $\operatorname{Aut}(A)$ is the group of permutations $\sigma$ of I so that $a_{\sigma(i), \sigma(j)}=a_{i, j}$ for $i, j \in \mathrm{I}$. By [KW, $\S 4.19]$ there exists a group monomorphism $\sigma \mapsto \tilde{\sigma}$ of $\operatorname{Aut}(A)$ into $\operatorname{Aut}(\mathfrak{g})$ such that $\tilde{\sigma}(\mathfrak{h})=\mathfrak{h}$ and

$$
\tilde{\sigma}\left(e_{i}\right)=e_{\sigma(i)} \quad \text { and } \quad \tilde{\sigma}\left(f_{i}\right)=f_{\sigma(i)},
$$

for $\sigma \in \operatorname{Aut}(A)$ and $i \in \mathrm{I}$. It is clear that $\sigma \mapsto \tilde{\sigma}$ is unique when $A$ has finite type, and this is also true when $A$ is affine (see Proposition 7.2.1 below). In any case, we fix a choice of the monomorphism $\sigma \mapsto \tilde{\sigma}$ and we use this map to identify $\operatorname{Aut}(A)$ as a subgroup of $\operatorname{Aut}(\mathfrak{g})$.

Next let $\omega$ be the unique automorphism of $\mathfrak{g}$ (the Chevalley automorphism of $\mathfrak{g}$ ) such that

$$
\omega\left(e_{i}\right)=-f_{i}, \quad \omega\left(f_{i}\right)=-e_{i} \quad \text { and } \quad \omega(h)=-h
$$

for $i \in \mathrm{I}$ and $h \in \mathfrak{h}$. Now $\omega$ has order 2 and it commutes with the automorphisms in $\operatorname{Aut}(A)$, so we may define the outer automorphism group of $\mathfrak{g}$ by

$$
\operatorname{Out}(A):= \begin{cases}\operatorname{Aut}(A), & \text { if } A \text { has finite type; } \\ \langle\omega\rangle \times \operatorname{Aut}(A), & \text { otherwise. }\end{cases}
$$

Then $\operatorname{Out}(A)$ is a finite subgroup of $\operatorname{Aut}(\mathfrak{g})$.

Next let $\operatorname{Aut}^{\mathrm{e}}(\mathfrak{g})=\left\langle\exp (\operatorname{ad}(x)) \mid x \in \mathfrak{g}_{\alpha}, \alpha \in \Delta^{\mathrm{re}}\right\rangle$ in $\operatorname{Aut}(\mathfrak{g})$. We also have $\operatorname{subgroups} \operatorname{Aut}(\mathfrak{g} ; \mathfrak{h}):=\{\sigma \in \operatorname{Aut}(\mathfrak{g}) \mid \sigma(h)=h$ for $h \in \mathfrak{h}\}$ and $\operatorname{Aut}\left(\mathfrak{g} ; \mathfrak{g}^{\prime}\right)=\{\sigma \in$ $\operatorname{Aut}(\mathfrak{g}) \mid \sigma(x)=x$ for $\left.x \in \mathfrak{g}^{\prime}\right\}$ of $\operatorname{Aut}(\mathfrak{g})$. Then $\operatorname{Aut}(\mathfrak{g} ; \mathfrak{h})$ normalizes Aut ${ }^{\mathrm{e}}(\mathfrak{g})$, while $\operatorname{Aut}\left(\mathfrak{g} ; \mathfrak{g}^{\prime}\right)$ centralizes both $\operatorname{Aut}^{\mathrm{e}}(\mathfrak{g})$ and $\operatorname{Aut}(\mathfrak{g} ; \mathfrak{h})$. Hence,

$$
\operatorname{Aut}^{0}(\mathfrak{g}):=\operatorname{Aut}^{\mathrm{e}}(\mathfrak{g}) \operatorname{Aut}(\mathfrak{g} ; \mathfrak{h}) \operatorname{Aut}\left(\mathfrak{g} ; \mathfrak{g}^{\prime}\right)
$$


is a subgroup of $\operatorname{Aut}(\mathfrak{g})$, which we call the inner automorphism group of $\mathfrak{g}$. We will recall below in Proposition 6.1.5 that $\operatorname{Aut}(\mathfrak{g})=\operatorname{Aut}^{0}(\mathfrak{g}) \rtimes \operatorname{Out}(A)$. If $\mathfrak{g}$ is finite dimensional, then $\operatorname{Aut}^{0}(\mathfrak{g})$ coincides with $\mathbf{G}_{\mathrm{ad}}(k)$ where $\mathbf{G}_{\mathrm{ad}}$ is the group of adjoint type corresponding to $\mathfrak{g}$. Furthermore, in that case, $\mathbf{G}_{\text {ad }}$ is the connected component of the identity of the algebraic group $\operatorname{Aut}(\mathfrak{g})$.

6.1.2. We have group homomorphisms

$$
\chi_{1}: \operatorname{Aut}(\mathfrak{g}) \mapsto \operatorname{Aut}\left(\mathfrak{g}^{\prime}\right) \quad \text { and } \quad \chi_{2}: \operatorname{Aut}\left(\mathfrak{g}^{\prime}\right) \mapsto \operatorname{Aut}\left(\overline{\mathfrak{g}^{\prime}}\right),
$$

where $\chi_{1}(v)=\left.v\right|_{\mathfrak{g}^{\prime}}$ for $v \in \operatorname{Aut}(\mathfrak{g})$ and $\chi_{2}(\tau)=\bar{\tau}$ for $\tau \in \operatorname{Aut}\left(\mathfrak{g}^{\prime}\right)$, and where $\bar{\tau}$ denotes the automorphism induced by $\tau$ on $\overline{\mathfrak{g}^{\prime}}=\mathfrak{g}^{\prime} / Z\left(\mathfrak{g}^{\prime}\right)$. Let

$$
\operatorname{Aut}^{0}\left(\mathfrak{g}^{\prime}\right)=\chi_{1}\left(\operatorname{Aut}^{0}(\mathfrak{g})\right) \text { and } \operatorname{Aut}^{0}\left(\overline{\mathfrak{g}^{\prime}}\right)=\left(\chi_{2} \circ \chi_{1}\right)\left(\operatorname{Aut}^{0}(\mathfrak{g})\right) .
$$

Also, it is easy to see that $\chi_{1}$ and $\chi_{2} \circ \chi_{1}$ restricted to $\operatorname{Out}(A)$ are injective, and we use these maps to identify $\operatorname{Out}(A)$ with a subgroup of $\operatorname{Aut}\left(\mathfrak{g}^{\prime}\right)$ and $\operatorname{Aut}\left(\overline{\mathfrak{g}^{\prime}}\right)$ respectively. In particular, we are regarding $\operatorname{Aut}(A)$ as a subgroup of $\operatorname{Aut}(\mathfrak{g}), \operatorname{Aut}\left(\mathfrak{g}^{\prime}\right)$ and $\operatorname{Aut}\left(\overline{\mathfrak{g}^{\prime}}\right)$.

Definition 6.1.3. We use the term diagram automorphism to refer to an automorphism of $\mathfrak{g}, \mathfrak{g}^{\prime}$ or $\overline{\mathfrak{g}^{\prime}}$ that lies in $\operatorname{Aut}(A)$.

6.1.4. There is a unique action of $\operatorname{Out}(A)$ on $Q$ such that $\nu\left(\alpha_{i}\right)=\alpha_{\nu(i)}$ and $\omega\left(\alpha_{i}\right)=-\alpha_{i}$ for all $\nu \in \operatorname{Aut}(A)$, and $i \in \mathrm{I}$. Then, with the identifications of 6.1.2, we have

$$
\sigma\left(\mathfrak{g}_{\alpha}\right)=\mathfrak{g}_{\sigma(\alpha)}, \quad \sigma\left(\left(\mathfrak{g}^{\prime}\right)_{\alpha}\right)=\left(\mathfrak{g}^{\prime}\right)_{\sigma(\alpha)} \quad \text { and } \quad \sigma\left(\left(\overline{\mathfrak{g}^{\prime}}\right)_{\alpha}\right)=\left(\overline{\mathfrak{g}^{\prime}}\right)_{\sigma(\alpha)}
$$

for $\sigma \in \operatorname{Out}(A)$ and $\alpha \in Q$.

The following result on the structure of $\operatorname{Aut}(\mathfrak{g}), \operatorname{Aut}\left(\mathfrak{g}^{\prime}\right)$ and $\operatorname{Aut}\left(\overline{\mathfrak{g}^{\prime}}\right)$ is due to Peterson and Kac [PK] (see also [ABP2, Prop. 7.3]).

Proposition 6.1.5. $\chi_{1}$ is surjective with kernel $\operatorname{Aut}\left(\mathfrak{g} ; \mathfrak{g}^{\prime}\right)$, and $\chi_{2}$ is an isomorphism. Furthermore

$$
\begin{aligned}
\operatorname{Aut}(\mathfrak{g}) & =\operatorname{Aut}^{0}(\mathfrak{g}) \rtimes \operatorname{Out}(A), \\
\operatorname{Aut}\left(\mathfrak{g}^{\prime}\right) & =\operatorname{Aut}^{0}\left(\mathfrak{g}^{\prime}\right) \rtimes \operatorname{Out}(A), \\
\operatorname{Aut}\left(\overline{\mathfrak{g}^{\prime}}\right) & =\operatorname{Aut}^{0}\left(\overline{\mathfrak{g}^{\prime}}\right) \rtimes \operatorname{Out}(A) .
\end{aligned}
$$

6.1.6. Next we let $p: \operatorname{Aut}(\mathfrak{g}) \rightarrow \operatorname{Out}(A), p^{\prime}: \operatorname{Aut}\left(\mathfrak{g}^{\prime}\right) \rightarrow \operatorname{Out}(A)$ and $\bar{p}: \operatorname{Aut}\left(\overline{\mathfrak{g}^{\prime}}\right) \rightarrow$ $\operatorname{Out}(A)$ be the projections onto the second factor relative to the decompositions in (7). Then we have the commutative diagram:

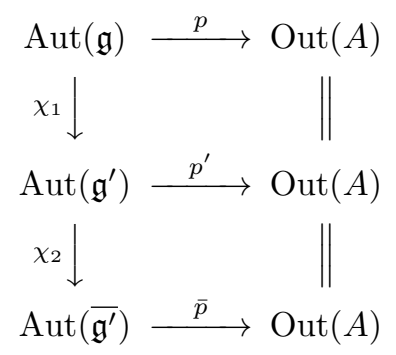


Definition 6.1.7. Suppose that $\sigma$ is an automorphism of $\mathfrak{g}, \mathfrak{g}^{\prime}$ or $\overline{\mathfrak{g}^{\prime}}$. We say that $\sigma$ is of first kind if $p(\sigma) \in \operatorname{Aut}(A), p^{\prime}(\sigma) \in \operatorname{Aut}(A)$ or $\bar{p}(\sigma) \in \operatorname{Aut}(A)$ respectively. Otherwise, we say that $\sigma$ is of second kind. ${ }^{3}$

Proposition 6.1.8. The group homomorphism $\chi_{1}$ has a section (namely a homomorphism that is a right inverse of $\left.\chi_{1}\right)$.

Proof. We use the notation and identifications of Sections 2.4 and 6.1. For convenience we set

$$
G=\operatorname{Aut}^{\mathrm{e}}(\mathfrak{g}), \quad \widetilde{H}=\operatorname{Aut}(\mathfrak{g} ; \mathfrak{h}) \quad \text { and } \quad K=\operatorname{Aut}\left(\mathfrak{g} ; \mathfrak{g}^{\prime}\right) .
$$

By Proposition 6.1.5, $\chi_{1}$ is an epimorphism with kernel $K$ and

$$
\operatorname{Aut}(\mathfrak{g})=\operatorname{Aut}^{0}(\mathfrak{g}) \rtimes \operatorname{Out}(A)=(G \widetilde{H} K) \rtimes \operatorname{Out}(A) .
$$

Note also that $\widetilde{H}$ normalizes $G$, so $G \widetilde{H}$ is a subgroup of $\operatorname{Aut}(\mathfrak{g})$. Further $\operatorname{Out}(A)$ normalizes $G \widetilde{H}$, so $(G \widetilde{H}) \rtimes \operatorname{Out}(A)$ is a subgroup of $\operatorname{Aut}(\mathfrak{g})$. So $\left.\chi_{1}\right|_{(G \widetilde{H}) \rtimes \operatorname{Out}(A)}$ : $(G \widetilde{H}) \rtimes \operatorname{Out}(A) \rightarrow \operatorname{Aut}\left(\mathfrak{g}^{\prime}\right)$ is an epimorphism with kernel $((G \widetilde{H}) \rtimes \operatorname{Out}(A)) \cap K$. Thus it suffices to show that $((G \widetilde{H}) \rtimes \operatorname{Out}(A)) \cap K=\{1\}$. Hence it suffices to show that

$$
(G \widetilde{H}) \cap K=\{1\} .
$$

Let $\Delta_{+}$be the set of positive roots of $\mathfrak{g}$; let $U_{+}$denote the subgroup of $G$ generated by the automorphisms of the form $\exp (\operatorname{ad}(x))$, where $x \in \mathfrak{g}_{\alpha}, \alpha \in \Delta^{\text {re }} \cap$ $\Delta_{+} ;$and let $\tilde{B}_{+}=U_{+} \widetilde{H}$. Then $\tilde{B}_{+}$is a subgroup of $G \widetilde{H}$ and

$$
G \widetilde{H}=\cup_{w \in W} \tilde{B}_{+} n_{w} \tilde{B}_{+},
$$

where the family $\left\{n_{w}\right\}_{w \in W}$ of elements of $G$ satisfies $n_{1}=1, n_{w}(\mathfrak{h})=\mathfrak{h}$ and $\left.n_{w}\right|_{\mathfrak{h}}=w$ for $w \in W$. (Here we are identifying $W$ as a subgroup of GL(h) as usual [MP, Lemma 5.1.2].) Indeed, this follows from the Bruhat decomposition for the derived group of $\mathfrak{g}$, since that group is mapped onto $G$ by the adjoint map [MP, Prop. 6.3.7].

Observe also from the definition of $\tilde{B}_{+}$that

$$
b \in \tilde{B}_{+} \Longrightarrow b\left(\mathfrak{n}_{+}\right) \subseteq \mathfrak{n}_{+} \text {and } b(h) \in h+\mathfrak{n}_{+} \text {for } h \in \mathfrak{h},
$$

where $\mathfrak{n}_{+}=\sum_{\alpha \in \Delta_{+}} \mathfrak{g}_{\alpha}$.

To show (9), suppose that $p \in(G \widetilde{H}) \cap K$. Then, by (10), we have

$$
p=b_{1} n_{w} b_{2},
$$

where $b_{1}, b_{2} \in \tilde{B}_{+}$and $w \in W$. Thus $n_{w}=b_{1}^{-1} p b_{2}^{-1}$. So for $h \in \mathfrak{h}^{\prime}$, we have $n_{w}(h) \in h+\mathfrak{n}_{+}$by (11). Thus $\left.w\right|_{\mathfrak{h}^{\prime}}=1$, and hence, by [MP, Cor. 5.2.1], $w=1$. Therefore, $p \in \tilde{B}_{+} \cap K$. Now it easy to see that the elements of $K$ stabilize $\mathfrak{h}$ (see for example [ABP2, Prop. 7.5(a)]). In particular, $p$ stabilizes $\mathfrak{h}$. So, since $p \in \tilde{B}_{+}$, it follows from (11) that $\left.p\right|_{\mathfrak{h}}=1$. Since $\mathfrak{g}=\mathfrak{g}^{\prime}+\mathfrak{h}$, we have $p=1$ as desired.

\footnotetext{
${ }^{3}$ Although we will not need this fact, one can show that this definition of first and second kind agrees with the usual one which is defined using the image of the positive Borel subalgebra under $\sigma[\mathrm{KW}, \S 4.6]$.
} 


\subsection{Loop algebras of symmetrizable Kac-Moody Lie algebras.}

6.2.1. If $\mu_{1}, \mu_{2} \in \operatorname{Out}(A)$, we write $\mu_{1} \sim \mu_{2}$ to mean that $\mu_{1}$ is conjugate to $\mu_{2}$ or $\mu_{2}^{-1}$ in $\operatorname{Out}(A)$.

Using the results of [ABP2], we can prove the following:

Theorem 6.2.2. Suppose that $\mathfrak{g}=\mathfrak{g}(A)$ is the Kac-Moody Lie algebra determined by an indecomposable symmetrizable GCM A.

(a) If $v_{1}$ and $v_{2}$ are finite order automorphisms of $\mathfrak{g}$, then $\mathrm{L}\left(\mathfrak{g}, v_{1}\right) \simeq \mathrm{L}\left(\mathfrak{g}, v_{2}\right)$ if and only if $p\left(v_{1}\right) \sim p\left(v_{2}\right)$.

(b) If $\tau_{1}$ and $\tau_{2}$ are finite order automorphisms of $\mathfrak{g}^{\prime}$, then $\mathrm{L}\left(\mathfrak{g}^{\prime}, \tau_{1}\right) \simeq \mathrm{L}\left(\mathfrak{g}^{\prime}, \tau_{2}\right)$ if and only if $p^{\prime}\left(\tau_{1}\right) \sim p^{\prime}\left(\tau_{2}\right)$.

(c) If $\sigma_{1}$ and $\sigma_{2}$ are finite order automorphisms of $\overline{\mathfrak{g}^{\prime}}$, then $\bar{p}\left(\sigma_{1}\right) \sim \bar{p}\left(\sigma_{2}\right)$ implies that $\mathrm{L}\left(\overline{\mathfrak{g}^{\prime}}, \sigma_{1}\right) \simeq \mathrm{L}\left(\overline{\mathfrak{g}^{\prime}}, \sigma_{2}\right)$.

Proof. (a): This is Theorem 9.3 of [ABP2].

(b): This is stated in [ABP2] as a corollary of (a). For the sake of completeness (since the proof is not entirely obvious) we include the necessary details here. By Proposition 6.1.8, we can choose $v_{i} \in \operatorname{Aut}(\mathfrak{g})$ of finite order so that $\chi_{i}\left(v_{i}\right)=\tau_{i}$ for $i=1,2$. Then $\mathrm{L}\left(\mathfrak{g}^{\prime}, \tau_{1}\right) \simeq \mathrm{L}\left(\mathfrak{g}^{\prime}, \tau_{2}\right)$ if and only if $\mathrm{L}\left(\mathfrak{g}, v_{1}\right) \simeq \mathrm{L}\left(\mathfrak{g}, v_{2}\right)$ (by $[\mathrm{ABP} 2$, Theorem 8.6]) which holds if and only if $p\left(v_{1}\right) \sim p\left(v_{2}\right)$ (by (a)). But, by (8), $p^{\prime}\left(\tau_{i}\right)=p\left(v_{i}\right)$ for $i=1,2$. So we have (b).

(c): Suppose that $\bar{p}\left(\sigma_{1}\right) \sim \bar{p}\left(\sigma_{2}\right)$. Since $\chi_{1}$ has a section and $\chi_{2}$ is an isomorphism, we can choose $v_{i}$ of finite order in $\operatorname{Aut}(\mathfrak{g})$ such that $\left(\chi_{2} \circ \chi_{1}\right)\left(v_{i}\right)=\sigma_{i}$ for $i=1,2$. Then, by (8), we have $p\left(v_{1}\right) \sim p\left(v_{2}\right)$, so $\mathrm{L}\left(\mathfrak{g}, v_{1}\right) \simeq \mathrm{L}\left(\mathfrak{g}, v_{2}\right)$ by (a). Thus, $\mathrm{L}\left(\mathfrak{g}, v_{1}\right)^{\prime} / \mathrm{Z}\left(\mathrm{L}\left(\mathfrak{g}, v_{1}\right)^{\prime}\right) \simeq \mathrm{L}\left(\mathfrak{g}, v_{2}\right)^{\prime} / \mathrm{Z}\left(\mathrm{L}\left(\mathfrak{g}, v_{2}\right)^{\prime}\right)$. So by Lemma 4.1.9, we have $\mathrm{L}\left(\overline{\mathfrak{g}^{\prime}}, \sigma_{1}\right) \simeq \mathrm{L}\left(\overline{\mathfrak{g}^{\prime}}, \sigma_{2}\right)$.

\section{Remark 6.2.3.}

(a) If $\mathfrak{g}$ is finite dimensional simple, then $\mathfrak{g}^{\prime}=\mathfrak{g}, \mathrm{Z}\left(\mathfrak{g}^{\prime}\right)=0, \overline{\mathfrak{g}^{\prime}} \simeq \mathfrak{g}$, Out $(A)=$ $\operatorname{Aut}(A)$, and the elements of $\operatorname{Aut}(A)$ are the classical diagram automorphisms.

(b) If $\mathfrak{g}$ is finite dimensional simple and $\mathbb{k}=\mathbb{C}$, Theorem 6.2.2 is a result of Kac. (See $[\mathrm{P} 1]$ and $[\mathrm{P} 2]$ for other generalizations of Kac's result.)

(c) If $\mathfrak{g}$ is affine and $\sigma_{1}$ and $\sigma_{2}$ are of first kind, we will see in Corollary 13.2.3 that the converse in Theorem 6.2.2(c) is also true.

\subsection{Loop algebras of finite dimensional simple algebras and the class $\mathbb{M}_{1}$.}

The following realization theorem for affine algebras is due to V. Kac [K1, Lemma 22]. In the untwisted case (see Corollary 6.3.2), it was proved independently by R. Moody [Mo, Thm. 2]. A detailed proof is given in [K2, $\S 7.4$ and $\S 8.3]$ in the complex case, and that proof also works in general.

Theorem 6.3.1 (Kac).

(a) If $\dot{\mathfrak{g}}$ is a finite dimensional simple Lie algebra of type $X_{k}$ and $\dot{\sigma}$ is a diagram automorphism of $\dot{\mathfrak{g}}$ of order $m$, then $\mathrm{L}(\dot{\mathfrak{g}}, \dot{\sigma}) \simeq \overline{\mathfrak{g}}^{\prime}$, where $\mathfrak{g}=\mathfrak{g}(A)$ is the Kac-Moody Lie algebra constructed from the affine GCM A of type $X_{k}^{(m)}$.

(b) Conversely, if $\mathfrak{g}=\mathfrak{g}(A)$, where $A$ is the affine $G C M$ of type $X_{k}^{(m)}$, then there is an isomorphism $\varphi$ from $\overline{\mathfrak{g}^{\prime}}$ onto a loop algebra $\mathrm{L}_{m}(\dot{\mathfrak{g}}, \dot{\sigma})$ contained in $\dot{\mathfrak{g}} \otimes S_{1}$, where $\dot{\mathfrak{g}}$ is a finite dimensional simple Lie algebra of type $X_{k}$ and $\dot{\sigma}$ is a diagram automorphism of $\dot{\mathfrak{g}}$ of order $m$. Moreover, this isomorphism can be chosen such 
that

$$
t_{1} \varphi\left(\left(\overline{\mathfrak{g}^{\prime}}\right)_{\alpha}\right)=\varphi\left(\left(\overline{\mathfrak{g}^{\prime}}\right)_{\alpha+m \delta}\right)
$$

for $\alpha \in Q$, where $t_{1}=z_{1}^{m}$ in $R_{1}=\mathbb{k}\left[t_{1}^{ \pm 1}\right]$ (see 4.1.6), and $\delta$ is the standard null root (see (13) below).

Corollary 6.3.2. A Lie algebra $\mathcal{L}$ is isomorphic to $\overline{\mathfrak{g}^{\prime}}$ for some untwisted affine Kac-Moody Lie algebra if and only if $\mathcal{L} \simeq \mathrm{L}(\dot{\mathfrak{g}}, 1)$ for some finite dimensional simple Lie algebra $\dot{\mathfrak{g}}$.

In the complex case, the equivalence of (a), (b), (c) and (d) in the following corollary is due to Kac.

Corollary 6.3.3. If $\mathcal{L}$ is a Lie algebra, the following statements are equivalent:

(a) $\mathcal{L} \in \mathbb{M}_{1}$.

(b) $\mathcal{L} \simeq \mathrm{L}(\dot{\mathfrak{g}}, \dot{\sigma})$ for some finite dimensional simple Lie algebra $\dot{\mathfrak{g}}$ and some diagram automorphism $\dot{\sigma}$ of $\dot{\mathfrak{g}}$.

(c) $\mathcal{L} \simeq \overline{\mathfrak{g}^{\prime}}$ for some affine Kac-Moody Lie algebra $\mathfrak{g}$.

(d) $\mathcal{L} \in \mathbb{I}_{1}$.

(e) $\mathcal{L} \in \mathbb{E}_{1}$.

Proof. First of all "(a) $\Rightarrow(\mathrm{b})$ " follows from Theorem 6.2.2(a) (applied to $\dot{\mathfrak{g}}$ ); while "(b) $\Rightarrow(\mathrm{a})$ " is trivial. Next (b) and (c) are equivalent by Theorem 6.3.1; (a) and (d) are equivalent by definition; and (c) and (e) are equivalent by Proposition 5.2.3(b).

Corollary 6.3.4. Suppose that $\mathfrak{g}=\mathfrak{g}(A)$, where $A$ is the affine $G C M$ of type $X_{k}^{(m)}$. Then $\overline{\mathfrak{g}^{\prime}}$ is prime, perfect and fgc; and the absolute type and relative type of $\overline{\mathfrak{g}^{\prime}}$ are respectively $X_{k}$ and the quotient type of $\mathfrak{g}$ (see 5.1.5).

Proof. This follows from Theorem 6.3.1(b), Propositions 4.4.3 and 4.4.4, and Theorem 5.5.2(a).

6.3.5. For each affine GCM $A$, the quotient type of $\mathfrak{g}=\mathfrak{g}(A)$ is calculated in $[\mathrm{K} 2$, Prop. 6.3]. We display the results in Table 1 below, where $\mathrm{X}_{k}^{(1)}$ denotes any one of the untwisted affine types. By Corollary 6.3.4, this table also displays the relative type of $\overline{\mathfrak{g}^{\prime}}$.

\begin{tabular}{|c|c|}
\hline$A$ & Quotient type of $\mathfrak{g}(A)$ \\
\hline $\mathrm{X}_{k}^{(1)}$ & $\mathrm{X}_{k}$ \\
\hline $\mathrm{A}_{k}^{(2)}, k \geq 2, k \neq 3$ & $\mathrm{BC}_{\frac{k}{2}}(k$ even $)$ or $\mathrm{C}_{\frac{k+1}{2}}(k$ odd $)$ \\
\hline $\mathrm{D}_{k}^{(2)}, k \geq 3$ & $\mathrm{~B}_{k-1}$ \\
\hline $\mathrm{D}_{4}^{(3)}$ & $\mathrm{G}_{2}$ \\
\hline $\mathrm{E}_{6}^{(2)}$ & $\mathrm{F}_{4}$ \\
\hline
\end{tabular}

TABLE 1 
Remark 6.3.6. Suppose that $\mathfrak{g}=\mathfrak{g}(A)$ is affine. If $A$ has type $\mathrm{X}_{k}^{(m)}$, using the labels from $[\mathrm{K} 2]$ as above, then by Corollary 6.3.4 the absolute type of $\overline{\mathfrak{g}^{\prime}}$ can be read from the label as $\mathrm{X}_{k}$. In [MP, $\left.\S 3.5\right]$ a different system is used to label affine matrices which allows one to read the quotient type of $\mathfrak{g}$, and hence also the relative type of $\overline{\mathfrak{g}^{\prime}}$, from the label in the same fashion. For example if $A$ has label $\mathrm{D}_{4}^{(3)}$ as above, the label for $A$ in $[\mathrm{MP}]$ is $\mathrm{G}_{2}^{(3)}$. Thus both systems convey important information about the Lie algebra $\overline{\mathfrak{g}^{\prime}}$. We will continue to use the labels from [K2] as discussed in Section 2.4.

The realization theorem leads to a classification of the algebras in $\mathbb{M}_{1}$ using the description of these algebras in Corollary 6.3.3(b).

\section{Corollary 6.3.7.}

(a) If $\dot{\sigma}_{i}$ is a diagram automorphism of a finite dimensional simple Lie algebra $\dot{\mathfrak{g}}_{i}, i=1,2$, then $\mathrm{L}\left(\dot{\mathfrak{g}}_{1}, \dot{\sigma}_{1}\right) \simeq \mathrm{L}\left(\dot{\mathfrak{g}}_{2}, \dot{\sigma}_{2}\right)$ implies that $\dot{\mathfrak{g}}_{1} \simeq \dot{\mathfrak{g}}_{2}$.

(b) If $\dot{\sigma}_{i}$ is a diagram automorphism of a finite dimensional simple Lie algebra $\dot{\mathfrak{g}}, i=1,2$, then $\mathrm{L}\left(\dot{\mathfrak{g}}, \dot{\sigma}_{1}\right) \simeq \mathrm{L}\left(\dot{\mathfrak{g}}, \dot{\sigma}_{2}\right)$ if and only if $\dot{\sigma}_{1}$ and $\dot{\sigma}_{2}$ are conjugate in the group of diagram automorphisms of $\dot{\mathfrak{g}}$.

Proof. This follows from the Peterson-Kac conjugacy theorem [PK, Thm. 2] and Theorem 6.3.1(a). (See also Remark 8.13 of [ABP2].) Another proof using Galois cohomology is given in [P2]. Alternatively, (a) follows from Proposition 4.4.4; and (b) follows from Theorem 6.2.2(a) (since every element of $\operatorname{Aut}(\mathfrak{g})$ is conjugate to its inverse).

Corollary 6.3.8. An algebra $\mathcal{L}$ is in $\mathbb{I}_{2}$ if and only if $\mathcal{L} \simeq \mathrm{L}\left(\overline{\mathfrak{g}^{\prime}}, \sigma\right)$ for some affine Lie algebra $\mathfrak{g}=\mathfrak{g}(A)$ and some finite order $\sigma \in \operatorname{Aut}(\mathfrak{g})$. Moreover, in that case, if $A$ has type $X_{k}^{(m)}$, then $\mathcal{L}$ has absolute type $X_{k}$.

Proof. The first statement follows from the definition of $\mathbb{I}_{2}$ and Corollary 6.3.3; and the second statement follows from Corollary 6.3.4 and Proposition 4.1.10.

\section{Automorphisms of affine algebras}

In Section 6.1, we discussed some general properties of automorphisms of symmetrizable Kac-Moody Lie algebras. In this section, we obtain some more detailed information in the affine case.

We assume throughout the section that $\mathfrak{g}=\mathfrak{g}(A)$ is the Kac-Moody Lie algebra determined by an affine $G C M A=\left(a_{i j}\right)_{i, j \in \mathrm{I}}$, where $\mathrm{I}=\{0, \ldots, \ell\}$ with $\ell \geq 1$.

\subsection{Notation and terminology for affine algebras.}

Before looking at automorphisms, we collect some notation, recall some terminology and discuss some basic properties of affine algebras. We will use this material throughout the rest of this paper.

7.1.1. In addition to the notation of Section 2.4, we use the following notation that is standard in the affine case [K2]. Choose relatively prime positive integers $a_{0}, \ldots, a_{\ell}$ and relatively prime positive integers $a_{0}^{\vee}, \ldots, a_{\ell}^{\vee}$ so that $A\left[a_{0}, \ldots, a_{\ell}\right]^{t}=0$ and $\left[a_{0}^{\vee}, \ldots, a_{\ell}^{\vee}\right] A=0$. Note that if $\nu \in \operatorname{Aut}(A)$, then one has

$$
a_{\nu(i)}=a_{i} \quad \text { and } \quad a_{\nu(i)}^{\vee}=a_{i}^{\vee}
$$


for $i \in \mathrm{I}$ [FSS, p. 47]. Let

$$
c=\sum_{i \in \mathrm{I}} a_{i}^{\vee} \alpha_{i}^{\vee} \quad \text { and } \quad \delta=\sum_{i \in \mathrm{I}} a_{i} \alpha_{i},
$$

in which case $\mathrm{Z}\left(\mathfrak{g}^{\prime}\right)=\mathbb{k} c$ and $\delta$ is a $\mathbb{Z}$-basis for the lattice of isotropic roots of $\mathfrak{g}$. Let $d \in \mathfrak{h}$ be a scaling element satisfying $\alpha_{i}(d)=\delta_{i, 0}$ for $i \in \mathrm{I}$, in which case

$$
\mathfrak{g}=\mathfrak{g}^{\prime} \oplus \mathbb{k} d \quad \text { and } \quad \mathfrak{h}=\mathfrak{h}^{\prime} \oplus \mathbb{k} d .
$$

Finally let $(\mid)$ be the normalized invariant form on $\mathfrak{g}[\mathrm{K} 2, \S 6.2]$; that is $(\mid)$ is the unique nondegenerate symmetric bilinear form on $\mathfrak{g}$ satisfying

$$
\left(\alpha_{i}^{\vee} \mid \alpha_{j}^{\vee}\right)=a_{j} a_{j}^{\vee} a_{i j}, \quad\left(\alpha_{i}^{\vee} \mid d\right)=\delta_{i, 0} a_{0} \quad \text { and } \quad(d \mid d)=0
$$

for $i, j \in \mathrm{I}$ (see [K2, $\S 6.1$ and 6.2$]$ where $c$ is denoted by $K$ ). Note in particular that

$$
\left(e_{i} \mid f_{i}\right)=\frac{a_{i}}{a_{i}^{\vee}} \quad \text { for } i \in \mathrm{I} .
$$

We also have $\left(\alpha_{i} \mid \alpha_{i}\right) \neq 0$ and

$$
a_{i j}=\frac{2\left(\alpha_{i} \mid \alpha_{j}\right)}{\left(\alpha_{i} \mid \alpha_{i}\right)}
$$

for $i, j \in \mathrm{I}[\mathrm{K} 2, \S 2.3]$.

Also, as for general EALAs in 5.1.5, we let

$$
V=\operatorname{span}_{\mathbb{Q}}(\Delta) .
$$

Then $\{\alpha\}_{i \in \mathrm{I}}$ is a $\mathbb{Q}$-basis for $V$. Note that the form $(\mid)$ restricted to $V$ takes rational values $[\mathrm{K} 2, \S 6.2-6.3]$ and we denote its restriction to $V$ also by $(\mid)$. Then, $(\mid): V \times V \rightarrow \mathbb{Q}$ is positive semi-definite with radical

$$
V^{0}:=\operatorname{rad}(V)=\mathbb{Q} \delta .
$$

We let $\bar{V}=V / V^{0}$ with canonical map ${ }^{-}: V \rightarrow \bar{V}$, in which case we have the induced positive definite form $(\mid): \bar{V} \times \bar{V} \rightarrow \mathbb{Q}$ on $\bar{V}$.

If $S \subseteq V$, we let

Then [K2, Prop. 5.10(c)]

$$
S^{\times}=\{\alpha \in S \mid(\alpha \mid \alpha) \neq 0\} .
$$

$$
\Delta^{\times}=\Delta^{\mathrm{re}}=\cup_{i \in \mathrm{I}} W \alpha_{i} .
$$

\subsection{Diagram automorphisms of affine algebras.}

We now recall from [Bau] that the embedding of $\operatorname{Aut}(A)$ into $\operatorname{Aut}(\mathfrak{g})$ discussed in 6.1.1 is unique in the affine case. This gives a unique interpretation of the notion of diagram automorphism.

Proposition 7.2.1. There exists a unique group homomorphism $\sigma \mapsto \tilde{\sigma}$ of $\operatorname{Aut}(A)$ into $\operatorname{Aut}(\mathfrak{g})$ such that $\tilde{\sigma}(\mathfrak{h})=\mathfrak{h}$,

$$
\tilde{\sigma}\left(e_{i}\right)=e_{\sigma(i)} \quad \text { and } \quad \tilde{\sigma}\left(f_{i}\right)=f_{\sigma(i)}
$$

for $\sigma \in \operatorname{Aut}(A)$ and $i \in \mathrm{I}$. This homomorphism is injective. Finally, the normalized invariant form ( | ) on $\mathfrak{g}$ is invariant under $\tilde{\sigma}$ for $\sigma \in \operatorname{Aut}(A)$.

Proof. We know that a homomorphism with the property indicated in the first sentence exists. Also, uniqueness follows from Lemma 2.2(b) of [Bau]. Furthermore, it is shown in the proof of that lemma that $\tilde{\sigma}(d) \in d+\mathfrak{h}^{\prime}$ for $\sigma \in \operatorname{Aut}(A)$.

Next it is clear that the map $\sigma \mapsto \tilde{\sigma}$ is injective, so all that remains is to prove the last statement. For this, let $\sigma \in \operatorname{Aut}(A)$, and define a new form $(\mid)^{\prime}$ on $\mathfrak{g}$ 
by $(x \mid y)^{\prime}=(\tilde{\sigma} x \mid \tilde{\sigma} y)$. Then, both $(\mid)$ and $(\mid)^{\prime}$ are invariant nondegenerate symmetric bilinear forms on $\mathfrak{g}$. Let

$$
\mathfrak{m}=\left\{x \in \mathfrak{g}:(x \mid y)^{\prime}=(x \mid y) \text { for all } y \in \mathfrak{g}\right\} .
$$

Then $\mathfrak{m}$ is an ideal of $\mathfrak{g}$, which we must show is $\mathfrak{g}$. First $e_{i} \in \mathfrak{m}$ for all $i$. To see this, observe that $e_{i}$ is orthogonal, using either form, to all root spaces of $\mathfrak{g}$ except $\mathfrak{g}_{-\alpha_{i}}$. Hence, it suffices to show that $\left(e_{i} \mid f_{i}\right)^{\prime}=\left(e_{i} \mid f_{i}\right)$, which follows from (12) and (14). So $e_{i} \in \mathfrak{m}$ and similarly $f_{i} \in \mathfrak{m}$. Thus, $\mathfrak{m}$ contains $\mathfrak{g}^{\prime}$. Finally, let $d^{\prime}=\sum_{i=0}^{|\sigma|-1} \tilde{\sigma}^{i} d$. Then, since $\tilde{\sigma}(d) \in d+\mathfrak{h}^{\prime}$, we see that $d^{\prime} \in \mathfrak{h} \backslash \mathfrak{h}^{\prime}$. Further, $\tilde{\sigma} d^{\prime}=d^{\prime}$ and so certainly $\left(d^{\prime} \mid d^{\prime}\right)^{\prime}=\left(d^{\prime} \mid d^{\prime}\right)$. But since $\mathfrak{m}$ contains $\mathfrak{g}^{\prime}$, we have $\left(x \mid d^{\prime}\right)^{\prime}=\left(x \mid d^{\prime}\right)$ for all $x \in \mathfrak{g}^{\prime}$. So $d^{\prime} \in \mathfrak{m}$, and we have $\mathfrak{m}=\mathfrak{g}$.

7.2.2. Suppose that $\sigma \in \operatorname{Aut}(A)$. As in 6.1.2 and 6.1.3, we will, whenever convenient, identify $\sigma$ with the automorphism $\tilde{\sigma},\left.\tilde{\sigma}\right|_{\mathfrak{g}^{\prime}}$ or $\overline{\left.\tilde{\sigma}\right|_{\mathfrak{g}^{\prime}}}$ of $\mathfrak{g}, \mathfrak{g}^{\prime}$ or $\overline{\mathfrak{g}^{\prime}}$ respectively.

\subsection{The kind of a finite order automorphism of an affine algebra.}

In the affine case, using Theorem 6.3.1(b) and the results of [ABP2.5], we can characterize the kind (as defined in Definition 6.1.7) of a finite order automorphism of $\overline{\mathfrak{g}^{\prime}}$ in terms of the centroid of the loop algebra $\mathrm{L}\left(\overline{\mathfrak{g}^{\prime}}, \sigma\right)$.

Proposition 7.3.1. Suppose that $\sigma$ is an automorphism of finite order of $\overline{\mathfrak{g}^{\prime}}$. Then, $\sigma$ is of first kind if and only if $\mathrm{C}\left(\mathrm{L}\left(\overline{\mathfrak{g}^{\prime}}, \sigma\right)\right) \simeq R_{2}$.

Proof. We use the isomorphism $\varphi$ in Theorem 6.3.1(b) to identify $\overline{\mathfrak{g}^{\prime}}=\mathrm{L}_{m_{1}}(\dot{\mathfrak{g}}, \dot{\sigma})$ in $\dot{\mathfrak{g}} \otimes S_{1}$, and we then identify $\mathrm{C}\left(\overline{\mathfrak{g}^{\prime}}\right)=R_{1}=\mathbb{k}\left[t_{1}^{ \pm 1}\right]$ as in Proposition 4.1.7, where $\dot{\sigma}$ has order $m_{1}$ and $t_{1}=z_{1}^{m_{1}}$. Then, $\mathrm{C}(\sigma) \in \operatorname{Aut}\left(R_{1}\right)$ and we have

$$
\left(C(\sigma)\left(t_{1}^{j}\right)\right)(x)=\sigma\left(t_{1}^{j}\left(\sigma^{-1} x\right)\right)
$$

for $x \in \overline{\mathfrak{g}^{\prime}}, j \in \mathbb{Z}$. Note that since $\mathrm{C}(\sigma) \in \operatorname{Aut}\left(R_{1}\right)$, we have

$$
\mathrm{C}(\sigma)\left(t_{1}\right) \in \mathbb{k}^{\times} t_{1}^{ \pm 1} .
$$

Let $m_{2}$ be a period for $\sigma$ and $\mathcal{L}=\mathrm{L}_{m_{2}}\left(\overline{\mathfrak{g}^{\prime}}, \sigma\right) \subseteq \overline{\mathfrak{g}^{\prime}} \otimes \mathbb{k}\left[z_{2}^{ \pm 1}\right]$. (We are using $z_{2}$ for the Laurent variable in this second loop construction to avoid possible confusion.) Then, by [ABP2.5, Prop. 4.11 and Prop. 4.13], we have $\mathrm{C}(\mathcal{L}) \simeq \mathrm{L}_{m_{2}}\left(\mathrm{C}\left(\overline{\mathfrak{g}^{\prime}}\right), \mathrm{C}(\sigma)\right)=$ $\mathrm{L}_{m_{2}}\left(R_{1}, \mathrm{C}(\sigma)\right)$, so

$$
\mathrm{C}(\mathcal{L}) \simeq \mathrm{L}_{m_{2}}\left(R_{1}, \mathrm{C}(\sigma)\right) \subseteq R_{1} \otimes \mathbb{k}\left[z_{2}^{ \pm 1}\right] .
$$

Therefore, by [ABP2.5, Lemma 9.1], we see that $\mathrm{C}(\mathcal{L}) \simeq R_{2}$ if and only if $t_{1} \otimes$ $z_{2}^{j} \in \mathrm{L}_{m_{2}}\left(R_{1}, \mathrm{C}(\sigma)\right)$ for some $j \in \mathbb{Z}$. But this last condition holds if and only if $\mathrm{C}(\sigma)\left(t_{1}\right) \in \zeta_{m_{2}}^{-j} t_{1}$ for some $j \in \mathbb{Z}$. So, since $\mathrm{C}(\sigma)$ has period $m_{2}$, we see that $\mathrm{C}(\mathcal{L}) \simeq R_{2}$ if and only if $\mathrm{C}(\sigma)\left(t_{1}\right) \in \mathbb{k}^{\times} t_{1}$. Therefore, it remains to show that

$$
\bar{p}(\sigma) \in \operatorname{Aut}(A) \Longleftrightarrow \mathrm{C}(\sigma)\left(t_{1}\right) \in \mathbb{k}^{\times} t_{1} .
$$

Now, by Proposition 6.1.5, we can write

$$
\sigma=\mu \rho \nu \omega^{i},
$$

where $\mu$ is a product of automorphisms of $\overline{\mathfrak{g}^{\prime}}$ of the form $\exp \left(\operatorname{ad}\left(x_{\alpha}\right)\right)$ with $x_{\alpha} \in\left(\overline{\mathfrak{g}^{\prime}}\right)_{\alpha}$ and $\alpha \in \Delta^{\mathrm{re}} ; \rho \in\left(\chi_{2} \circ \chi_{1}\right)(\operatorname{Aut}(\mathfrak{g}, \mathfrak{h})) ; \nu \in \operatorname{Aut}(A) ;$ and $i=0$ or 1 . Then, $\bar{p}(\sigma) \in \operatorname{Aut}(A)$ if and only if $i=1$. So, to prove (17), it suffices to show that

$$
\mathrm{C}(\mu)\left(t_{1}\right) \in \mathbb{k}^{\times} t_{1}, \quad \mathrm{C}(\rho)\left(t_{1}\right) \in \mathbb{k}^{\times} t_{1}, \quad \mathrm{C}(\nu)\left(t_{1}\right) \in \mathbb{k}^{\times} t_{1} \text { and } \mathrm{C}(\omega)\left(t_{1}\right) \in \mathbb{k}^{\times} t_{1}^{-1} .
$$


First, $\mu$ commutes with the action of $\mathrm{C}\left(\overline{\mathfrak{g}^{\prime}}\right)$, and hence $\mathrm{C}(\mu)\left(t_{1}\right)=t_{1}$. Next, if $\alpha \in \Delta$, we have $\rho\left(\left(\overline{\mathfrak{g}^{\prime}}\right)_{\alpha}\right)=\left(\overline{\mathfrak{g}^{\prime}}\right)_{\alpha}$. Hence for $\alpha \in \Delta$,

$$
\left(C(\rho)\left(t_{1}\right)\right)\left(\overline{\mathfrak{g}^{\prime}}\right)_{\alpha}=\rho\left(t_{1}\left(\overline{\mathfrak{g}^{\prime}}\right)_{\alpha}\right)=\rho\left(\left(\overline{\mathfrak{g}^{\prime}}\right)_{\alpha+m_{1} \delta}\right)=\left(\overline{\mathfrak{g}^{\prime}}\right)_{\alpha+m_{1} \delta} .
$$

So $C(\rho)\left(t_{1}\right) \notin \mathbb{k}^{\times} t_{1}^{-1}$, and thus $C(\rho)\left(t_{1}\right) \in \mathbb{k}^{\times} t_{1}$. Next $\nu(\delta)=\delta$ and $\nu\left(\left(\overline{\mathfrak{g}^{\prime}}\right)_{\alpha}\right)=$ $\left(\overline{\mathfrak{g}^{\prime}}\right)_{\nu(\alpha)}$ for $\alpha \in \Delta$. So calculating as above, we get $\left(C(\nu)\left(t_{1}\right)\right)\left(\overline{\mathfrak{g}^{\prime}}\right)_{\alpha}=\left(\overline{\mathfrak{g}^{\prime}}\right)_{\alpha+m_{1} \delta}$ for $\alpha \in \Delta$, and therefore $C(\nu)\left(t_{1}\right) \in \mathbb{k}^{\times} t_{1}$. Next $\omega(\delta)=-\delta$ and $\omega\left(\left(\overline{\mathfrak{g}^{\prime}}\right)_{\alpha}\right)=\left(\overline{\mathfrak{g}^{\prime}}\right)_{-\alpha}$ for $\alpha \in \Delta$. So, we get $\left(C(\omega)\left(t_{1}\right)\right)\left(\overline{\bar{g}^{\prime}}\right)_{\alpha}=\left(\overline{\mathfrak{g}^{\prime}}\right)_{\alpha-m_{1} \delta}$ for $\alpha \in \Delta$, hence $C(\omega)\left(t_{1}\right) \notin \mathbb{k}^{\times} t_{1}$, and therefore $C(\omega)\left(t_{1}\right) \in \mathbb{k}^{\times} t_{1}^{-1}$.

\section{LoOP ALGEBRAS OF AFFine ALGEBRAS RELATIVE TO DIAGRAM ROTATIONS}

Our goal in the next two sections is to obtain detailed information about $\mathrm{L}\left(\overline{\mathfrak{g}^{\prime}}, \sigma\right)$, where $\mathfrak{g}=\mathfrak{g}(A)$ is an affine algebra and $\sigma \in \operatorname{Aut}(A)$.

We say that $\sigma$ is transitive if the group $\langle\sigma\rangle$ acts transitively on I. Observe that by the classification of affine diagrams, $\sigma$ is transitive if and only if $\mathfrak{g}$ is of type $\mathrm{A}_{\ell}^{(1)}$, where $\ell \geq 1$, and $\sigma$ has order $\ell+1$ in the rotation group of the diagram.

We will consider two overlapping cases:

The rotation case: $\mathfrak{g}$ is of type $\mathrm{A}_{\ell}^{(1)}$, where $\ell \geq 1$ and $\sigma$ is in the rotation group of the diagram. (When $\ell=1, \sigma=(1)$ or $(01)$.)

The nontransitive case: $\sigma$ is not transitive.

Note that by the preceding observation every $\sigma$ is covered by one of these two cases.

In this section, we determine the structure of $\mathrm{L}\left(\overline{\mathfrak{g}^{\prime}}, \sigma\right)$ in the rotation case. We will consider the nontransitive case in the next section.

\subsection{The map $\iota_{m}$.}

8.1.1. (The map $\iota_{m}$ ) We next introduce a map $\iota_{m}: \mathbb{Z}_{m} \rightarrow \mathbb{Z}_{m}$ for $m \geq 1$, which will appear in our description of the structure of $\mathrm{L}\left(\overline{\mathfrak{g}^{\prime}}, \sigma\right)$ (Theorem 8.3.1). To do this, we begin by defining for each positive divisor $g$ of $m$ the set

$$
U(m, g)=\left\{\bar{k} \in \mathbb{Z}_{m} \mid \operatorname{gcd}(k, m)=g\right\} .
$$

(As is usual, we interpret $\operatorname{gcd}(0, m)$ as $m$.) So, in particular, $U(m, m)=\{\overline{0}\}$, and $U(m):=U(m, 1)$ is the group of units of the ring $\mathbb{Z}_{m}$. (Our convention is that $\overline{0}$ is a unit in $\mathbb{Z}_{1}=\{\overline{0}\}$.) Now

$$
\mathbb{Z}_{m}=\bigcup_{g \mid m} U(m, g) \text { (disjoint); }
$$

and, for each $g \mid m$, we have the bijection

$$
U\left(\frac{m}{g}\right) \stackrel{\cdot g}{\rightarrow} U(m, g)
$$

given by $\bar{k} \mapsto \overline{k g}$. We let $\iota_{m}: \mathbb{Z}_{m} \rightarrow \mathbb{Z}_{m}$ be the unique map such that, for each positive divisor $g$ of $m$, we have $\iota_{m}(U(m, g)) \subseteq U(m, g)$ and the diagram

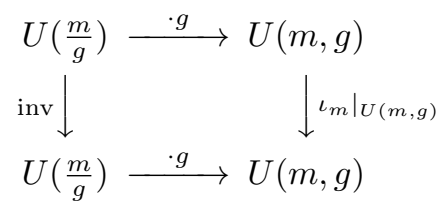

commutes, where inv is the inversion map on $U\left(\frac{m}{g}\right)$. It is clear from this definition that $\iota_{m}^{2}=1$ and hence $\iota_{m}$ is a bijection. 


\subsection{Some associative loop algebras.}

Our goal now is to realize certain associative loop algebras as matrix algebras over quantum tori. We use the notation $d_{m}(u), p_{m}(u), D_{m}(u)$ and $P_{m}(u)$ of 4.5.4.

Lemma 8.2.1. Suppose that $m \geq 1, \theta$ has order $m$ in $\mathbb{k}^{\times}$, and $q \in \mathbb{Z}$. Then, using the notation of Definition 4.1.1,

$$
\mathrm{L}_{m}\left(\mathrm{M}_{m}\left(R_{1}\right), P_{m}\left(t_{1}\right)^{q}, \theta\right) \simeq \mathrm{L}_{\boldsymbol{m}}\left(\mathrm{M}_{m}(\mathbb{k}),\left(D_{m}(\theta), P_{m}(1)^{q}\right), \boldsymbol{\theta}\right),
$$

where $\boldsymbol{m}=(m, m)$ and $\boldsymbol{\theta}=(\theta, \theta)$.

Proof. We identify $\mathrm{M}_{m}(\mathbb{k}) \otimes S_{1}=\mathrm{M}_{m}\left(S_{1}\right)$, where $S_{1}=\mathbb{k}\left[z_{1}^{ \pm 1}\right]$. Then

$$
\mathrm{L}_{m}\left(\mathrm{M}_{m}(\mathbb{k}), D_{m}(\theta), \theta\right)=\mathrm{M}_{m}\left(S_{1}\right)^{D_{m}(\theta) \otimes \eta} .
$$

where $\eta \in \operatorname{Aut}\left(S_{1}\right)$ satisfies $\eta\left(z_{1}\right)=\theta^{-1} z_{1}$ and $\mathrm{M}_{m}\left(S_{1}\right)^{D_{m}(\theta) \otimes \eta}$ is the algebra of fixed points of $D_{m}(\theta) \otimes \eta$ in $\mathrm{M}_{m}\left(S_{1}\right)$. Similarly, we have

$$
\mathrm{L}_{m}\left(\mathrm{M}_{m}(\mathbb{k}), 1, \theta\right)=\mathrm{M}_{m}\left(S_{1}\right)^{1 \otimes \eta}=\mathrm{M}_{m}\left(R_{1}\right),
$$

where $R_{1}=\mathbb{k}\left[t_{1}^{ \pm 1}\right]$ with $t_{1}=z_{1}^{m}$. But setting

$$
\varphi=D_{m}\left(z_{1}\right) \in \operatorname{Aut}\left(\mathrm{M}_{m}\left(S_{1}\right)\right)
$$

we have

$$
\begin{aligned}
\varphi(1 \otimes \eta) \varphi^{-1} & =\operatorname{Ad}\left(d_{m}\left(z_{1}\right)\right)(1 \otimes \eta) \operatorname{Ad}\left(d_{m}\left(z_{1}\right)^{-1}\right) \\
& =\operatorname{Ad}\left(d_{m}\left(z_{1}\right)\right) \operatorname{Ad}\left((1 \otimes \eta) d_{m}\left(z_{1}\right)^{-1}\right)(1 \otimes \eta) \\
& =\operatorname{Ad}\left(d_{m}\left(z_{1}\right) d_{m}\left(\eta z_{1}\right)^{-1}\right)(1 \otimes \eta)=D_{m}(\theta) \otimes \eta .
\end{aligned}
$$

It follows that $\varphi$ restricts to an isomorphism

$$
\varphi: \mathrm{L}_{m}\left(\mathrm{M}_{m}(\mathbb{k}), 1, \theta\right) \stackrel{\sim}{\rightarrow} \mathrm{L}_{m}\left(\mathrm{M}_{m}(\mathbb{k}), D_{m}(\theta), \theta\right) .
$$

So using Remark 4.2.2, we have

$$
\begin{aligned}
& \mathrm{L}_{\boldsymbol{m}}\left(\mathrm{M}_{m}(\mathbb{k}),\left(D_{m}(\theta), P_{m}(1)^{q}\right), \boldsymbol{\theta}\right) \simeq \mathrm{L}_{m}\left(\mathrm{~L}_{m}\left(\mathrm{M}_{m}(\mathbb{k}), D_{m}(\theta), \theta\right), P_{m}(1)^{q}, \theta\right) \\
& \simeq \mathrm{L}_{m}\left(\mathrm{~L}_{m}\left(\mathrm{M}_{m}(\mathbb{k}), 1, \theta\right), \varphi^{-1} P_{m}(1)^{q} \varphi, \theta\right) .
\end{aligned}
$$

But $\varphi^{-1} P_{m}(1) \varphi=\operatorname{Ad}\left(d_{m}\left(z_{1}\right)^{-1} p_{m}(1) d_{m}\left(z_{1}\right)\right)$, and a direct calculation shows that $d_{m}\left(z_{1}\right)^{-1} p_{m}(1) d_{m}\left(z_{1}\right)=z_{1}^{-1} p_{m}\left(z_{1}^{m}\right)$. So $\varphi^{-1} P_{m}(1) \varphi=P_{m}\left(z_{1}^{m}\right)$. Thus,

$$
\begin{aligned}
\mathrm{L}_{\boldsymbol{m}}\left(\mathrm{M}_{m}(\mathbb{k}),\left(D_{m}(\theta), P_{m}(1)^{q}\right), \boldsymbol{\theta}\right) & \simeq \mathrm{L}_{m}\left(\mathrm{~L}_{m}\left(\mathrm{M}_{m}(\mathbb{k}), 1, \theta\right), P_{m}\left(z_{1}^{m}\right)^{q}, \theta\right) \\
& =\mathrm{L}_{m}\left(\mathrm{M}_{m}\left(R_{1}\right), P_{m}\left(t_{1}\right)^{q}, \theta\right) .
\end{aligned}
$$

Proposition 8.2.2. Suppose that $m \geq 1, \theta$ has order $m$ in $\mathbb{k}^{\times}$and $q \in \mathbb{Z}$. Then

$$
\mathrm{L}\left(\mathrm{M}_{m}\left(R_{1}\right), P_{m}\left(t_{1}\right)^{q}, \theta\right) \simeq M_{\operatorname{gcd}(q, m)}\left(Q\left(\theta^{\iota_{m}(\bar{q})}\right)\right),
$$

where $\theta^{\iota_{m}(\bar{q})}$ has the evident well-defined interpretation.

Proof. By Lemma 8.2.1 and Lemma 4.5.6(a), we have

$$
\mathrm{L}\left(\mathrm{M}_{m}\left(R_{1}\right), P_{m}\left(t_{1}\right), \theta\right) \simeq Q(\theta) .
$$

Since $\iota_{m}(\overline{1})=\overline{1}$, this proves $(19)$ when $q=1$.

We next suppose that $q=g$, where $g$ is a positive divisor of $m$. Since $\iota_{m}(\bar{g})=\bar{g}$, we must show that

$$
\mathrm{L}_{m}\left(\mathrm{M}_{m}\left(R_{1}\right), P_{m}\left(t_{1}\right)^{g}, \theta\right) \simeq \mathrm{M}_{g}\left(Q\left(\theta^{g}\right)\right) .
$$


To see this, we regard matrices in $\mathrm{M}_{m}\left(R_{1}\right)$ as elements in $\operatorname{End}_{R_{1}}\left(R_{1}^{m}\right)$ by means of the natural left action on column vectors. Let $\left(x_{1}, \ldots, x_{m}\right)$ be the standard ordered $R_{1}$-basis for $R_{1}^{m}$ and let $k=\frac{m}{g}$. Then, relative to the new ordered $R_{1}$-basis

$$
\left(x_{1}, x_{1+g}, \ldots, x_{1+(k-1) g}, x_{2}, x_{2+g}, \ldots, x_{2+(k-1) g}, \ldots, x_{g}, x_{g+g}, \ldots, x_{g+(k-1) g}\right)
$$

of $R_{1}^{m}, p_{m}\left(t_{1}\right)^{g}$ has matrix

$$
p_{m, g}\left(t_{1}\right):=\operatorname{diag}\left(p_{k}\left(t_{1}\right), \ldots, p_{k}\left(t_{1}\right)\right)
$$

in block diagonal form with $g$ diagonal $k \times k$-blocks. So

$$
\mathrm{L}_{m}\left(\mathrm{M}_{m}\left(R_{1}\right), P_{m}\left(t_{1}\right)^{g}, \theta\right) \simeq \mathrm{L}_{m}\left(\mathrm{M}_{m}\left(R_{1}\right), P_{m, g}\left(t_{1}\right), \theta\right),
$$

where $P_{m, g}\left(t_{1}\right)=\operatorname{Ad}\left(p_{m, g}\left(t_{1}\right)\right)$. It follows that

$$
\mathrm{L}_{m}\left(\mathrm{M}_{m}\left(R_{1}\right), P_{m}\left(t_{1}\right)^{g}, \theta\right) \simeq \mathrm{M}_{g}\left(\mathrm{~L}_{m}\left(\mathrm{M}_{k}\left(R_{1}\right), P_{k}\left(t_{1}\right), \theta\right)\right) .
$$

But using Lemma 4.1.2(c) and (20), we have

$$
\mathrm{L}_{m}\left(M_{k}\left(R_{1}\right), P_{k}\left(t_{1}\right), \theta\right) \simeq \mathrm{L}_{k}\left(M_{k}\left(R_{1}\right), P_{k}\left(t_{1}\right), \theta^{g}\right) \simeq Q\left(\theta^{g}\right) .
$$

So we have (21).

Finally, suppose that $q \in \mathbb{Z}$ is arbitrary, and let $g=\operatorname{gcd}(m, q), k=\frac{m}{g}$ and $r=\frac{q}{g}$. Now $\operatorname{gcd}(r, k)=1$. Hence, since the map $\bar{s} \mapsto \bar{s}$ from $U(m)$ to $U(k)$ is surjective, we can choose $s \in \mathbb{Z}$ satisfying

$$
\operatorname{gcd}(s, m)=1 \quad \text { and } \quad s r \equiv 1 \quad(\bmod k) .
$$

Then

$$
\begin{array}{rlrl}
\mathrm{L}_{m}\left(\mathrm{M}_{m}\left(R_{1}\right), P_{m}\left(t_{1}\right)^{q}, \theta\right) & \simeq \mathrm{L}_{k}\left(\mathrm{M}_{m}\left(R_{1}\right), P_{m}\left(t_{1}\right)^{q}, \theta^{g}\right) & & (\text { by Lemma 4.1.2(c)) } \\
& =\mathrm{L}_{k}\left(\mathrm{M}_{m}\left(R_{1}\right),\left(P_{m}\left(t_{1}\right)^{g}\right)^{r}, \theta^{g}\right) & \\
& \simeq \mathrm{L}_{k}\left(\mathrm{M}_{m}\left(R_{1}\right), P_{m}\left(t_{1}\right)^{g},\left(\theta^{g}\right)^{s}\right) & & (\text { by Lemma 4.1.2(b)) } \\
& \simeq \mathrm{L}_{m}\left(\mathrm{M}_{m}\left(R_{1}\right), P_{m}\left(t_{1}\right)^{g}, \theta^{s}\right) & & (\text { by Lemma 4.1.2(c) }) \\
& \simeq \mathrm{M}_{g}\left(Q\left(\theta^{s g}\right)\right) & & (\text { by }(21)) \\
& \simeq \mathrm{M}_{g}\left(Q\left(\theta^{\iota}(\bar{q})\right) .\right. &
\end{array}
$$

\subsection{The structure of $\mathrm{L}\left(\overline{\mathfrak{g}^{\prime}}, \rho^{q}\right)$.}

We now assume that $\mathfrak{g}=\mathfrak{g}(A)$, where $A$ is the affine $G C M$ of type $A_{\ell}^{(1)}, \ell \geq 1$. We enumerate the fundamental roots $\alpha_{0}, \alpha_{1}, \ldots, \alpha_{\ell}$ in cyclic order around the Dynkin diagram as in $[\mathrm{K} 2, \S 4.8]$; and we use the notation of Section 7 for affine algebras. Let

$$
\rho=(0,1, \ldots, \ell) \in \operatorname{Aut}(A)
$$

so $\rho$ is a rotation of the Dynkin diagram by 1 position. Then a general rotation of the Dynkin diagram has the form $\rho^{q}$, where $q \in \mathbb{Z}$.

We now prove a theorem that gives realizations of the loop algebras $\mathrm{L}\left(\overline{\mathfrak{g}^{\prime}}, \rho^{q}\right)$ as multiloop algebras (see (23)) and as matrix algebras over quantum tori (see (24)).

Theorem 8.3.1. Suppose that $q \in \mathbb{Z}$. Then, we have

$$
\begin{aligned}
& \mathrm{L}\left(\overline{\mathfrak{g}^{\prime}}, \rho^{q}\right) \simeq \mathrm{L}\left(\mathfrak{s l}_{\ell+1}\left(R_{1}\right), P_{\ell+1}\left(t_{1}\right)^{q}\right), \\
& \mathrm{L}\left(\overline{\mathfrak{g}^{\prime}}, \rho^{q}\right) \simeq \mathrm{L}\left(\mathfrak{s l}_{\ell+1}(\mathbb{k}),\left(D_{\ell+1}\left(\zeta_{\ell+1}\right), P_{\ell+1}(1)^{q}\right)\right)
\end{aligned}
$$


and

$$
\mathrm{L}\left(\overline{\mathfrak{g}^{\prime}}, \rho^{q}\right) \simeq \mathfrak{s l}_{\operatorname{gcd}(q, \ell+1)}\left(Q\left(\zeta_{\ell+1}^{\ell \ell+1}(\bar{q})\right)\right) .
$$

Proof. By Theorem 6.3.1 (with $m=1$ and $t_{1}=z_{1}$ ), we can identify

$$
\overline{\mathfrak{g}^{\prime}}=\mathfrak{s l}_{\ell+1}(\mathbb{k}) \otimes R_{1}=\mathfrak{s l}_{\ell+1}\left(R_{1}\right) .
$$

Furthermore, by [K2, Thm. 7.4], this can be done with

$$
\overline{e_{0}}=t_{1} E_{\ell+1,1}, \quad \overline{f_{0}}=t_{1}^{-1} E_{1, \ell+1}, \quad \overline{e_{i}}=E_{i, i+1}, \quad \overline{f_{i}}=E_{i+1, i}
$$

for $1 \leq i \leq \ell$, where $E_{i j}$ denotes the $(i, j)$-matrix unit in $\mathrm{M}_{\ell+1}\left(R_{1}\right)$. Now, a direct calculation shows that $P_{\ell+1}\left(t_{1}^{-1}\right)\left(\overline{e_{i}}\right)=\overline{e_{i+1}}$ and $P_{\ell+1}\left(t_{1}^{-1}\right)\left(\overline{f_{i}}\right)=\overline{f_{i+1}}$ for $0 \leq i \leq \ell$, where the subscripts are interpreted modulo $\ell+1$. So $\rho=P_{\ell+1}\left(t_{1}^{-1}\right)$. Therefore $\mathrm{L}\left(\overline{\mathfrak{g}^{\prime}}, \rho^{q}\right)=\mathrm{L}\left(\mathfrak{s}_{\ell+1}\left(R_{1}\right), P_{\ell+1}\left(t_{1}^{-1}\right)^{q}\right)$. Replacing $t_{1}$ by $t_{1}^{-1}$ we have $(22)$.

Now taking the derived algebras of both sides of (18) (with $m=\ell+1$ and $\left.\theta=\zeta_{\ell+1}\right)$ we see, using Lemma 4.1.9(a), that the right hand sides of (22) and (23) are isomorphic. Similarly, taking the derived algebras both sides of (19), we see that the right hand sides of (22) and (24) are isomorphic.

Corollary 8.3.2. If $q \in \mathbb{Z}, \mathrm{L}\left(\overline{\mathfrak{g}^{\prime}}, \rho^{q}\right) \in \mathbb{M}_{2}$. Moreover, if $q$ is relatively prime to $\ell+1$, then the relative type of $\mathrm{L}\left(\overline{\mathfrak{g}^{\prime}}, \rho^{q}\right)$ is $A_{0}$; in other words $\mathrm{L}\left(\overline{\mathfrak{g}^{\prime}}, \rho^{q}\right)$ is anisotropic.

Proof. This follows from (24) and Proposition 4.5.8(b).

\section{LoOP ALGEBRAS OF AFFine ALGEBRAS RELATIVE TO NONTRANSITIVE DIAGRAM AUTOMORPHISMS}

Suppose that $\mathfrak{g}=\mathfrak{g}(A)$ is the Kac-Moody Lie algebra constructed from an affine $G C M A=\left(a_{i j}\right)_{i, j \in \mathrm{I}}$, and suppose that $\sigma \in \operatorname{Aut}(A), m$ is a positive integer and $\sigma^{m}=1$. We continue with the notation of Section 7 for affine algebras.

In this section, we recall results from [ABP1] that show that if $\sigma$ is not transitive, $\mathrm{L}\left(\overline{\mathfrak{g}^{\prime}}, \sigma\right)$ is the centreless core of an EALA which is constructed as the affinization of $\mathfrak{g}$ relative to $\sigma$. We use this and Theorem 5.5.2 about EALAs to give a description of the relative type of $\mathrm{L}\left(\overline{\mathfrak{g}^{\prime}}, \sigma\right)$ in terms of $\sigma$ and the root system of $\mathfrak{g}$.

\subsection{Affinization.}

Initially we do not assume that $\sigma$ is nontransitive.

9.1.1. Let

$$
\operatorname{Aff}_{m}(\mathfrak{g}, \sigma):=\mathrm{L}_{m}(\mathfrak{g}, \sigma) \oplus \mathbb{k} \tilde{c} \oplus \mathbb{k} \tilde{d}
$$

as a vector space, where $\tilde{c}, \tilde{d} \neq 0$, and define a skew-symmetric product on $\operatorname{Aff}_{m}(\mathfrak{g}, \sigma)$ by

$$
\begin{aligned}
& {\left[x \otimes z_{1}^{i}+r_{1} \tilde{c}+r_{2} \tilde{d}, y \otimes z_{1}^{j}+s_{1} \tilde{c}+s_{2} \tilde{d}\right]=} \\
& {[x, y] \otimes z_{1}^{i+j}+j r_{2} y \otimes z^{j}-i s_{2} x \otimes z^{i}+i \delta_{i+j, 0}(x, y) \tilde{c}}
\end{aligned}
$$

for $i, j \in \mathbb{Z}, x \in \mathfrak{g}^{\bar{\imath}}, y \in \mathfrak{g}^{\bar{\jmath}}, r_{1}, r_{2}, s_{1}, s_{2} \in \mathbb{k}$. One checks easily that $\operatorname{Aff}_{m}(\mathfrak{g}, \sigma)$ is a Lie algebra which we call the affinization of $\mathfrak{g}$ relative to $\sigma$. We define a form $(\mid)$ on $\operatorname{Aff}_{m}(\mathfrak{g}, \sigma)$ by

$$
\left(x \otimes z_{1}^{i}+r_{1} \tilde{c}+r_{2} \tilde{d} \mid y \otimes z_{1}^{j}+s_{1} \tilde{c}+s_{2} \tilde{d}\right)=\delta_{i+j, 0}(x \mid y)+r_{1} s_{2}+r_{2} s_{1} .
$$


Then, since $\sigma$ preserves the form on $\mathfrak{g}$ by Proposition 7.2.1, it follows that $(\mid)$ is a nondegenerate symmetric bilinear form on $\operatorname{Aff}_{m}(\mathfrak{g}, \sigma)$ [ABP1, Lemma 3.2]. Finally, let $\mathcal{H}=\left(\mathfrak{h}^{\sigma} \otimes 1\right) \oplus \mathbb{k} \tilde{c} \oplus \mathbb{k} \tilde{d}$, in which case $\mathcal{H}$ is an abelian subalgebra of $\operatorname{Aff}_{m}(\mathfrak{g}, \sigma)$.

To discuss the root system of $\operatorname{Aff}_{m}(\mathfrak{g}, \sigma)$ we need some further notation.

9.1.2. We have $\sigma(\mathfrak{h})=\mathfrak{h}$, so $\sigma$ acts on $\mathfrak{h}^{*}$ by the inverse dual action:

$$
(\sigma(\alpha))(h)=\alpha\left(\sigma^{-1}(h)\right)
$$

for $\alpha \in \mathfrak{h}^{*}, h \in \mathfrak{h}$. Then

$$
\sigma(\Delta)=\Delta, \quad \text { so } \quad \sigma(V)=V .
$$

Next, since $\sigma$ has finite order, we have

$$
V=V^{\sigma} \oplus(1-\sigma)(V),
$$

where $V^{\sigma}$ denotes the fixed point set of $\sigma$ acting on $V$. Let $\pi_{\sigma}: V \rightarrow V^{\sigma}$ be the projection of $V$ onto $V^{\sigma}$ relative to the decomposition (29).

Proposition 9.1.3. Suppose that $\mathfrak{g}=\mathfrak{g}(A)$ is an affine Kac-Moody Lie algebra with root system $\Delta, \sigma \in \operatorname{Aut}(A)$ is not transitive, and $\sigma^{m}=1$. Then the triple $\left(\operatorname{Aff}_{m}(\mathfrak{g}, \sigma),(\mid), \mathcal{H}\right)$ defined in 9.1.1 is an EALA of nullity 2 which is discrete if $\mathbb{k}=\mathbb{C}$. Moreover, the centreless core of this EALA is isomorphic to $\mathrm{L}_{m}\left(\overline{\mathfrak{g}^{\prime}}, \sigma\right)$.

Finally, $\overline{\pi_{\sigma}(\Delta)}$ is an irreducible finite root system in $\overline{V^{\sigma}}$ and the quotient type of $\left(\mathrm{Aff}_{m}(\mathfrak{g}, \sigma),(\mid), \mathcal{H}\right)$ is equal to the type of the finite root system $\overline{\pi_{\sigma}(\Delta)}$.

Proof. If $\mathbb{k}=\mathbb{C}$, these statements are proved in [ABP1]. (See Theorem 4.8, Lemma 3.57 and Equation (3.48) of [ABP1].) The result for general $\mathbb{k}$ is proved by straightforward modification of the arguments given there. In particular, instead of working with the $\mathbb{R}$-spans of the root systems for $\mathfrak{g}$ and $\operatorname{Aff}_{m}(\mathfrak{g}, \sigma)$, one works with the $\mathbb{Q}$ spans. Also, Lemma 3.33 of [ABP1] must be replaced by the statement that the group generated by the isotropic roots of $\operatorname{Aff}_{m}(\mathfrak{g}, \sigma)$ is finitely generated. This is easily checked using $[\mathrm{ABP} 1,(3.31)]$. The remaining changes are evident, so we omit further explanation.

\subsection{Relative type in the nontransitive case.}

Theorem 9.2.1. Suppose that $\mathfrak{g}=\mathfrak{g}(A)$ is an affine Kac-Moody Lie algebra and $\sigma \in \operatorname{Aut}(A)$ is not transitive. Then $\mathrm{L}\left(\overline{\mathfrak{g}^{\prime}}, \sigma\right) \in \mathbb{M}_{2} \cap \mathbb{E}_{2}$, and the relative type of $\mathrm{L}\left(\overline{\mathfrak{g}^{\prime}}, \sigma\right)$ is equal to the type of the finite root system $\overline{\pi_{\sigma}(\Delta)}$.

Proof. Let $\mathcal{L}=\mathrm{L}\left(\overline{\mathfrak{g}^{\prime}}, \sigma\right)$. Then $\mathcal{L}$ is fgc by Corollary 6.3.8 and Proposition 4.4.3, and $\mathcal{L} \in \mathbb{E}_{2}$ by Proposition 9.1.3. Our conclusion now follows from Proposition 9.1.3 and Theorem 5.5.2.

9.2.2. We will return in Section 12 to study the finite root system $\overline{\pi_{\sigma}(\Delta)}$ and see how to calculate its type.

\section{Characterizations of algebras in $\mathbb{M}_{2}$}

\subsection{Characterizations.}

Our first main theorem gives three characterizations of the algebras in $\mathbb{M}_{2}$.

Theorem 10.1.1. For a Lie algebra $\mathcal{L}$, the following statements are equivalent:

(a) $\mathcal{L} \in \mathbb{M}_{2}$. 
(b) $\mathcal{L} \simeq \mathrm{L}\left(\overline{\mathfrak{g}^{\prime}}, \sigma\right)$, where $\mathfrak{g}$ is an untwisted affine Kac-Moody Lie algebra and $\sigma$ is a diagram automorphism of $\overline{\mathfrak{g}^{\prime}}$.

(c) $\mathcal{L} \simeq \mathrm{L}\left(\overline{\mathfrak{g}^{\prime}}, \sigma\right)$, where $\mathfrak{g}$ is an affine Kac-Moody Lie algebra and $\sigma$ is a finite order automorphism of $\overline{\mathfrak{g}^{\prime}}$ of first kind.

(d) $\mathcal{L} \in \mathbb{I}_{2}$ and $\mathrm{C}(\mathcal{L})$ is isomorphic to $R_{2}$.

Proof. (c) and (d) are equivalent by Corollary 6.3.8 and Proposition 7.3.1. It remains therefore to show that (a), (b) and (c) are equivalent.

"(a) $\Rightarrow(\mathrm{b})$ ": We have $\mathcal{L} \simeq \mathrm{L}(\dot{\mathfrak{g}}, \boldsymbol{\sigma})$, where $\dot{\mathfrak{g}}$ is a finite dimensional simple Lie algebra and $\boldsymbol{\sigma}=\left(\sigma_{1}, \sigma_{2}\right)$ is a pair of commuting finite order automorphisms of $\dot{\mathfrak{g}}$. If $P=\left[\begin{array}{ll}a & b \\ c & d\end{array}\right] \in \mathrm{GL}_{2}(\mathbb{Z})$, we write $\boldsymbol{\sigma}^{P}=\left(\sigma_{1}^{a} \sigma_{2}^{c}, \sigma_{1}^{b} \sigma_{2}^{d}\right)$. Then, $\mathrm{L}\left(\dot{\mathfrak{g}}, \boldsymbol{\sigma}^{P}\right) \simeq \mathrm{L}(\dot{\mathfrak{g}}, \boldsymbol{\sigma})$ [GP1, Lemma 5.3], so we can replace $\boldsymbol{\sigma}$ by $\boldsymbol{\sigma}^{P}$ as needed.

Next $\dot{\mathfrak{g}}=\mathfrak{g}(\dot{A})$ for some GCM $\dot{A}$ of finite type, so, by Proposition 6.1.5, we have $\operatorname{Aut}(\dot{\mathfrak{g}})=\operatorname{Aut}^{0}(\dot{\mathfrak{g}}) \rtimes \operatorname{Aut}(\dot{A})$, since $\operatorname{Out}(\dot{A})=\operatorname{Aut}(\dot{A})$. We let $\dot{p}: \operatorname{Aut}(\dot{\mathfrak{g}}) \rightarrow \operatorname{Aut}(\dot{A})$ be the projection onto the second factor relative to this decomposition.

We claim that for suitable $P=\left[\begin{array}{ll}a & b \\ c & d\end{array}\right] \in \mathrm{GL}_{2}(\mathbb{Z})$, we have

$$
\dot{p}\left(\sigma_{1}^{a} \sigma_{2}^{c}\right)=1 \text {. }
$$

Now $\operatorname{Aut}(\dot{A})$ is isomorphic to $S_{3}, \mathbb{Z} / 2 \mathbb{Z}$ or $\{1\}$. So, since $\dot{p}\left(\sigma_{1}\right)$ and $\dot{p}\left(\sigma_{2}\right)$ commute, one of these is a power of the other. If $\dot{p}\left(\sigma_{2}\right)=\dot{p}\left(\sigma_{1}\right)^{k}$, then (30) holds with $P=\left[\begin{array}{cc}-k & 1 \\ 1 & 0\end{array}\right]$. On the other hand, if $\dot{p}\left(\sigma_{1}\right)=\dot{p}\left(\sigma_{2}\right)^{k}$, then (30) holds with $P=\left[\begin{array}{cc}1 & 0 \\ -k & 1\end{array}\right]$. This demonstrates the claim.

So we can replace $\boldsymbol{\sigma}$ by $\boldsymbol{\sigma}^{P}$ and assume that $\dot{p}\left(\sigma_{1}\right)=1$. Hence, using Theorem 6.2.2(a) (applied to $\dot{\mathfrak{g}})$ and Corollary 6.3.2, we have $\mathrm{L}\left(\dot{\mathfrak{g}}, \sigma_{1}\right) \simeq \mathrm{L}\left(\dot{\mathfrak{g}}, \dot{p}\left(\sigma_{1}\right)\right)=$ $\mathrm{L}(\dot{\mathfrak{g}}, 1) \simeq \overline{\mathfrak{g}^{\prime}}$, where $\mathfrak{g}=\mathfrak{g}(A)$ is an untwisted affine algebra. Therefore,

$$
\mathcal{L} \simeq \mathrm{L}(\dot{\mathfrak{g}}, \boldsymbol{\sigma}) \simeq \mathrm{L}\left(\mathrm{L}\left(\dot{\mathfrak{g}}, \sigma_{1}\right),\left.\sigma_{2} \otimes 1\right|_{\mathrm{L}\left(\dot{\mathfrak{g}}, \sigma_{1}\right)}\right) \simeq \mathrm{L}\left(\overline{\mathfrak{g}^{\prime}}, \tau\right),
$$

for some automorphism $\tau$ of $\overline{\mathfrak{g}^{\prime}}$ of finite order.

Next, by Proposition 4.1.7, the centroid $\mathrm{C}(\mathcal{L})$ of $\mathcal{L}$ is isomorphic to $R_{2}$. Thus, by Proposition 7.3.1, $\tau$ is of first kind. So using the notation of 6.1.6, $\bar{p}(\tau) \in \operatorname{Aut}(A)$. Finally, by Theorem 6.2.2(c), we have $\mathcal{L} \simeq \mathrm{L}\left(\overline{\mathfrak{g}^{\prime}}, \tau\right) \simeq \mathrm{L}\left(\overline{\mathfrak{g}^{\prime}}, \bar{p}(\tau)\right)=\mathrm{L}\left(\overline{\mathfrak{g}^{\prime}}, \sigma\right)$.

"(b) $\Rightarrow$ (c)": This is trivial.

"(c) $\Rightarrow$ (a)": Suppose $\mathcal{L} \simeq \mathrm{L}\left(\overline{\mathfrak{g}^{\prime}}, \sigma\right)$, where $\mathfrak{g}=\mathfrak{g}(A)$ is an affine Kac-Moody Lie algebra and $\sigma$ is a finite order automorphism of $\overline{\mathfrak{g}^{\prime}}$ of first kind. By Theorem 6.2.2(c) again, we have $\mathcal{L} \simeq \mathrm{L}\left(\overline{\mathfrak{g}^{\prime}}, \bar{p}(\sigma)\right)$; so replacing $\sigma$ by $\bar{p}(\sigma)$, we can assume that $\sigma \in \operatorname{Aut}(A)$. Then $\mathcal{L} \in \mathbb{M}_{2}$ by Corollary 8.3.2 and Theorem 9.2.1.

Remark 10.1.2. If $\mathcal{L} \simeq \mathrm{L}\left(\overline{\mathfrak{g}^{\prime}}, \sigma\right)$, where $\sigma$ is a diagram automorphism of an affine algebra of type $\mathrm{X}_{k}^{(1)}$, it follows from Theorem 10.1.1 and Proposition 4.1.10 that $\mathcal{L} \simeq \mathrm{L}(\dot{\mathfrak{g}}, \boldsymbol{\tau})$, where $\boldsymbol{\tau}$ is a 2-tuple of commuting finite order automorphisms of a finite dimensional simple Lie algebra $\dot{\mathfrak{g}}$ of type $\mathrm{X}_{k}$. It would be interesting to obtain a simple algorithm to find such a $\boldsymbol{\tau}$ in each case. This may be possible with a careful examination of the steps in the proof of Theorem 10.1.1.

We now look more closely at the relationship between the class $\mathbb{M}_{2}$ and the classes $\mathbb{E}_{2}$ and $\mathbb{I}_{2}$. This is presented in a sequence of corollaries of Theorem 10.1.1.

Corollary 10.1.3. For a Lie algebra $\mathcal{L}$, the following statements are equivalent:

(a) $\mathcal{L} \in \mathbb{M}_{2} \cap \mathbb{E}_{2}$.

(b) $\mathcal{L} \in \mathbb{I}_{2} \cap \mathbb{E}_{2}$. 
(c) $\mathcal{L} \in \mathbb{E}_{2}$ and $\mathcal{L}$ is fgc.

(d) $\mathcal{L} \in \mathbb{M}_{2}$ and $\mathcal{L}$ is isotropic.

(e) $\mathcal{L} \simeq \mathrm{L}\left(\overline{\mathfrak{g}^{\prime}}, \sigma\right)$, where $\mathfrak{g}$ is an untwisted affine Kac-Moody Lie algebra and $\sigma$ is a nontransitive diagram automorphism of $\overline{\mathfrak{g}^{\prime}}$.

Proof. "(a) $\Rightarrow$ (b)" holds since $\mathbb{M}_{2} \subseteq \mathbb{I}_{2}$, "(b) $\Rightarrow$ (c)" follows from Proposition 4.4.3, and "(c) $\Rightarrow$ (d)" follow from Theorem 5.5.2.

"(d) $\Rightarrow(\mathrm{e})$ ": By Theorem 10.1.1, we have $\mathcal{L} \simeq \mathrm{L}\left(\overline{\mathfrak{g}^{\prime}}, \sigma\right)$, where $\mathfrak{g}=\mathfrak{g}(A)$ is an untwisted affine algebra and $\sigma \in \operatorname{Aut}(A)$. Then, since $\mathcal{L}$ is isotropic, it follows from Corollary 8.3.2 that $\sigma$ is not transitive.

"(e) $\Rightarrow$ (a)": This holds by Theorem 9.2.1.

Corollary 10.1.4. For a Lie algebra $\mathcal{L}$, the following statements are equivalent:

(a) $\mathcal{L} \in \mathbb{M}_{2} \backslash \mathbb{E}_{2}$.

(b) $\mathcal{L} \in \mathbb{M}_{2}$ and $\mathcal{L}$ is anisotropic.

(c) $\mathcal{L} \simeq \mathrm{L}\left(\overline{\mathfrak{g}^{\prime}}, \sigma\right)$, where $\mathfrak{g}$ is an untwisted affine Kac-Moody Lie algebra and $\sigma$ is a transitive diagram automorphism of $\overline{\mathfrak{g}^{\prime}}$.

(d) $\mathcal{L} \simeq \mathfrak{s l}_{1}(Q(\theta))$, where $\theta \neq 1$ is a root of unity in $\mathbb{k}^{\times}$.

Proof. First "(a) $\Rightarrow$ (c)" by Theorem 10.1.1 and Corollary 10.1.3; second "(c) $\Rightarrow$ (b)" by Corollary 8.3.2; and third "(b) $\Rightarrow$ (a)" by Corollary 10.1.3. Therefore, (a), (b) and (c) are equivalent. Finally (d) and (c) are equivalent by (24), since any root of unity $\neq 1$ in $\mathbb{k}^{\times}$equals $\zeta_{\ell+1}^{r}$ for some $\ell \geq 1$ and some $r$ relatively prime to $\ell+1$.

Remark 10.1.5. We have described the algebras in $\mathbb{M}_{2} \backslash \mathbb{E}_{2}$ as matrix algebras. This can also be done for the algebras in $\mathbb{E}_{2} \backslash \mathbb{M}_{2}$ using the coordinatization theorems for algebras in $\mathbb{E}_{n}$ (see 5.3.6). In nullity 2 , it turns out that the only algebras in $\mathbb{E}_{2}$ that are not fgc, and hence not in $\mathbb{M}_{2}$, are the algebras isomorphic to $\mathfrak{s l}_{g}(Q(\theta))$, for some $g \geq 2$ and some $\theta$ of infinite order in $\mathbb{k}^{\times}$. (See [Neh2] for a more general theorem.) We will not use this fact in this paper except to include it in Figure 1 below.

Corollary 10.1.6. For a Lie algebra $\mathcal{L}$, the following statements are equivalent:

(a) $\mathcal{L} \in \mathbb{I}_{2} \backslash \mathbb{M}_{2}$.

(b) $\mathcal{L} \simeq \mathrm{L}\left(\overline{\mathfrak{g}^{\prime}}, \omega \sigma\right)$, where $\mathfrak{g}$ is an untwisted affine Kac-Moody Lie algebra, $\omega$ is the Chevalley automorphism and $\sigma$ is a diagram automorphism of $\overline{\mathfrak{g}^{\prime}}$.

(c) $\mathcal{L} \simeq \mathrm{L}\left(\overline{\mathfrak{g}^{\prime}}, \sigma\right)$, where $\mathfrak{g}$ is an affine Kac-Moody Lie algebra and $\sigma$ is a finite order automorphism of second kind of $\overline{\mathfrak{g}^{\prime}}$.

Proof. "(a) $\Rightarrow$ (b)" By Corollary 6.3.8, $\mathcal{L} \simeq \mathrm{L}\left(\overline{\mathfrak{g}^{\prime}}, \sigma\right)$ for some affine Lie algebra $\mathfrak{g}=\mathfrak{g}(A)$ and some finite order $\sigma \in \operatorname{Aut}(\mathfrak{g})$. By Theorem 6.2.2(c), we can assume that $\sigma \in \operatorname{Out}(A)$. Finally, $\sigma \notin \operatorname{Aut}(A)$, by Theorem 10.1.1.

"(b) $\Rightarrow(\mathrm{c})$ " is trivial.

"(c) $\Rightarrow$ (a)" By Corollary 6.3.8, $\mathcal{L} \in \mathbb{I}_{2}$. Further, if $\mathcal{L} \in \mathbb{M}_{2}$, then $\mathrm{C}(\mathcal{L}) \simeq R_{2}$ by Proposition 4.1.7, which implies that $\sigma$ is of first kind by Proposition 7.3.1.

10.1.7. Much of the information from this section is summarized in Figure 1 below. In the figure, $\mathbb{M}_{2}$ has a bold line border, $\mathbb{I}_{2}$ is the tall vertical rectangle, and $\mathbb{E}_{2}$ is the wide horizontal rectangle. 


\begin{tabular}{|c|c|}
\hline $\begin{array}{l}\mathbb{I}_{2} \backslash \mathbb{M}_{2} \\
=\left\{\mathrm{L}\left(\overline{\mathfrak{g}}^{\prime}, \omega \sigma\right) \mid \mathfrak{g} \text { untwisted affine, } \sigma \in \operatorname{Aut}(A)\right\} \\
=\left\{\text { algebras in } \mathbb{I}_{2} \text { with centroid } \nsucceq R_{2}\right\}\end{array}$ & \\
\hline $\begin{array}{l}\mathbb{M}_{2} \backslash \mathbb{E}_{2} \\
=\left\{\mathrm{L}\left(\overline{\mathfrak{g}^{\prime}}, \sigma\right) \mid \mathfrak{g} \text { untwisted affine, }\right. \\
=\{\in \operatorname{Aut}(A) \text { transitive }\} \\
=\left\{\text { anisotropic algebras in } \mathbb{M}_{2}\right\} \\
=\left\{\mathfrak{s l}_{1}(Q(\theta)) \mid \theta \text { a root of unity } \neq 1\right\}\end{array}$ & $\mathbb{M}_{2}$ \\
\hline 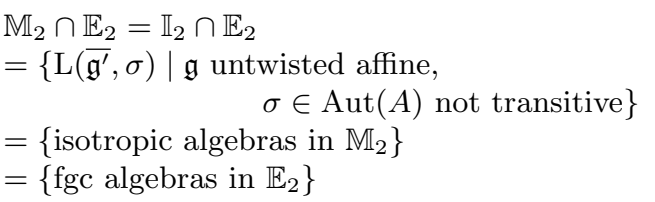 & $\begin{array}{l}\mathbb{E}_{2} \backslash \mathbb{M}_{2} \\
=\text { non-fgc algebras in } \mathbb{E}_{2} \\
=\left\{\mathfrak{s l}_{g}(Q(\theta)) \mid g \geq 2,\right. \\
\quad \quad \theta \text { not a root of unity }\}\end{array}$ \\
\hline
\end{tabular}

Figure 1 . The classes $\mathbb{M}_{2}, \mathbb{I}_{2}$ and $\mathbb{E}_{2}$

10.1.8. There is another interesting class of algebras related to multiloop algebras which has been investigated in [GP1] using cohomological methods. This is the class, which we denote here by $\mathbb{F}_{n}$, of $R_{n}$-forms of finite dimensional simple Lie algebras. (If $\mathfrak{g}$ is an algebra, an $R_{n}$-form of $\mathfrak{g}$ is an algebra $\mathcal{L}$ over $R_{n}$ such that $\mathcal{L} \otimes_{R_{n}} S \simeq_{S} \mathfrak{g} \otimes S$ for some faithfully flat and finitely presented extension $S / R_{n}$ of unital commutative associative algebras over $\mathbb{k}$.) For any $n \geq 1, \mathbb{F}_{n}$ contains $\mathbb{M}_{n}$ [GP1, §5.1]; if $n=1, \mathbb{F}_{1}=\mathbb{M}_{1}[\mathrm{P} 2]$, so $\mathbb{F}_{1}=\mathbb{M}_{1}=\mathbb{I}_{1}=\mathbb{E}_{1}$; but already if $n=2$, an example of B. Margaux shows that $\mathbb{F}_{2}$ properly contains $\mathbb{M}_{2}$ [GP1, Example 5.7]. Furthermore, algebras in $\mathbb{F}_{2}$ are fgc and have centroids isomorphic to $R_{2}$ [GP1, Lemma 4.6], so $\mathbb{F}_{2} \cap \mathbb{I}_{2}=\mathbb{M}_{2}$ and $\mathbb{F}_{2} \cap \mathbb{E}_{2}=\mathbb{M}_{2} \cap \mathbb{E}_{2}$. We will not discuss the class $\mathbb{F}_{n}$ further here, and instead refer the reader to [GP1] for more information.

\section{ISOMORPHISM CONDITIONS FOR MATRIX ALGEBRAS OVER QUANTUM TORI}

In the next two sections we develop the results we need to determine when two algebras of the form $\mathrm{L}\left(\overline{\mathfrak{g}^{\prime}}, \sigma\right)$, where $\mathfrak{g}$ is an affine algebra and $\sigma$ is a diagram automorphism of $\overline{\mathfrak{g}^{\prime}}$, are isomorphic. We do this for the rotation case in this section and for the nontransitive case in the next section.

Recall that $R_{2}=\mathbb{k}\left[t_{1}^{ \pm 1}, t_{2}^{ \pm 1}\right]$. We let

$$
K_{2}=\mathbb{k}\left(t_{1}, t_{2}\right)
$$

be the quotient field of $R_{2}$; that is $K_{2}$ is the field of rational functions in the variables $t_{1}, t_{2}$ over $\mathbb{k}$.

Recall also from 4.5.3 that if $\theta$ is an element of $\mathbb{k}^{\times}$of finite order $m$ and $Q(\theta)$ is the quantum torus determined by $\theta$ with distinguished generators $x_{1}, x_{2}, x_{1}^{-1}, x_{2}^{-1}$, then we have identified $R_{2}$ with the centre of $Q(\theta)$ by setting $t_{1}=x_{1}^{m}$ and $t_{2}=x_{2}^{m}$.

11.1. Comparing $Q(\theta)$ and $Q\left(\theta^{-1}\right)$.

Suppose that $\theta \in \mathbb{k}^{\times}$has order $m$. 
11.1.1. Note that $Q(\theta) \simeq Q\left(\theta^{-1}\right)$ under the isomorphism that exchanges the distinguished generators $x_{1}$ and $x_{2}$. However, it is not true that $Q(\theta) \simeq_{R_{2}} Q\left(\theta^{-1}\right)$ unless $m=1$ or 2 , as we'll see below in Lemma 11.3.1.

11.1.2. If $\mathcal{A}$ is an arbitrary algebra, we denote the opposite algebra of $\mathcal{A}$, with underlying vector space $\mathcal{A}$ and multiplication $(x, y) \mapsto y x$, by $\mathcal{A}^{\text {op }}$.

Lemma 11.1.3. Suppose that $g \geq 1$. Then

$$
\begin{gathered}
\mathrm{M}_{g}(Q(\theta)) \simeq \simeq_{R_{2}} \mathrm{M}_{g}\left(Q\left(\theta^{-1}\right)\right)^{o p} \\
\mathfrak{g l}_{g}(Q(\theta)) \simeq_{R_{2}} \mathfrak{g l}_{g}\left(Q\left(\theta^{-1}\right)\right) \quad \text { and } \quad \mathfrak{s l}_{g}(Q(\theta)) \simeq_{R_{2}} \mathfrak{s l}_{g}\left(Q\left(\theta^{-1}\right)\right) .
\end{gathered}
$$

Proof. Let $*$ denote the product in $Q\left(\theta^{-1}\right)^{\mathrm{op}}$. Then in $Q\left(\theta^{-1}\right)^{\mathrm{op}}$, we have $x_{1} * x_{2}=$ $x_{2} x_{1}=\theta x_{1} x_{2}=\theta x_{2} * x_{1}$. Hence, we have a $\mathbb{k}$-algebra isomorphism of $Q(\theta)$ onto $Q\left(\theta^{-1}\right)^{\text {op }}$ with $x_{i} \mapsto x_{i}$. It is clear that this is an $R_{2}$-algebra isomorphism, so $Q(\theta) \simeq_{R_{2}} Q\left(\theta^{-1}\right)^{\text {op }}$. Thus,

$$
\mathrm{M}_{g}(Q(\theta)) \simeq_{R_{2}} \mathrm{M}_{g}\left(Q\left(\theta^{-1}\right)^{\mathrm{op}}\right) \simeq_{R_{2}} \mathrm{M}_{g}\left(Q\left(\theta^{-1}\right)\right)^{\mathrm{op}},
$$

where the last isomorphism is the transpose map; and

$$
\mathfrak{g l}_{g}(Q(\theta)) \simeq_{R_{2}} \mathfrak{g l}_{g}\left(Q\left(\theta^{-1}\right)^{\mathrm{op}}\right) \simeq_{R_{2}} \mathfrak{g l}_{g}\left(Q\left(\theta^{-1}\right)\right),
$$

where the last isomorphism is additive inversion. Taking derived algebras now finishes the proof.

Lemma 11.1.4. If $\varphi \in \operatorname{Aut}\left(R_{2}\right)$, then there exists a $\mathbb{k}$-algebra isomorphism of $Q(\theta)$ onto either $Q(\theta)$ or $Q\left(\theta^{-1}\right)$ that extends $\varphi$.

Proof. It is well known that there exists a unique $P=\left[\begin{array}{ll}p_{11} & p_{12} \\ p_{21} & p_{22}\end{array}\right] \in \mathrm{GL}_{2}(\mathbb{Z})$ such that

$$
\varphi\left(t_{i}\right) \sim t_{1}^{p_{i 1}} t_{2}^{p_{i 2}}
$$

for $i=1,2$, where $\sim$ means that the first element is obtained from the second by multiplying by an element of $\mathbb{k}^{\times}$. Let $\varepsilon=\operatorname{det}(P) \in\{ \pm 1\}$. We will show that $\varphi$ extends to an isomorphism from $Q(\theta)$ to $Q\left(\theta^{\varepsilon}\right)$. Let $\psi_{\text {ex }}: Q(\theta) \rightarrow Q\left(\theta^{-1}\right)$ be the isomorphism mentioned in 11.1.1. Replacing $\varphi$ by $\left.\psi_{\mathrm{ex}}\right|_{R_{2}} \varphi$ if necessary, we can assume that $\varepsilon=1$. But then, since $P \in \mathrm{SL}_{2}(\mathbb{Z})$, there exists $\psi_{P} \in \operatorname{Aut}(Q(\theta))$ such that $\psi_{P}\left(x_{i}\right) \sim x_{1}^{p_{i 1}} x_{2}^{p_{i 2}}$ for $i=1,2$ [Neeb, Lemmas IV.3 and IV.6]. Then $\psi_{P}\left(t_{i}\right) \sim t_{i}^{p_{i 1}} t_{2}^{p_{i 2}}$, so $\left(\psi_{P}^{-1} \varphi\right)\left(t_{i}\right) \sim t_{i}$ for $i=1,2$. Hence, replacing $\varphi$ by $\left.\psi_{P}^{-1}\right|_{R_{2}} \varphi$, we can assume that $\varphi\left(t_{i}\right) \sim t_{i}$ for $i=1,2$. Since $\mathbb{k}^{\times}=\left(\mathbb{k}^{\times}\right)^{m}$, the proof in this case is clear.

\subsection{Cyclic algebras and quantum tori.}

Suppose that $\theta \in \mathbb{k}^{\times}$has order $m$.

11.2.1. (Cyclic algebras) Suppose that $F$ is a unital commutative associative $\mathbb{k}$-algebra and $u_{1}, u_{2}$ are units in $F$. We let

$$
\mathcal{A}=\left(u_{1}, u_{2} ; m, F, \theta\right)
$$

denote the algebra over $F$ presented by the generators $y_{1}, y_{2}$ subject to the relations $y_{1} y_{2}=\zeta y_{2} y_{1}, y_{1}^{m}=u_{1}$ and $y_{2}^{m}=u_{2}$. We call $\mathcal{A}$ the cyclic algebra over $F$ determined by $u_{1}, u_{2}$ and $\theta$, and we call $y_{1}, y_{2}$ the distinguished generators of $\mathcal{A}$ over $F$. It is easy to see that $\mathcal{A}$ is a free $F$-module of rank $m^{2}$ with basis $\left\{y_{1}^{k_{1}} y_{2}^{k_{2}} \mid 0 \leq k_{1}, k_{2} \leq m-1\right\}$. If $F$ is a field, it is known that $\mathcal{A}$ is a central simple algebra over $F$ [D, Theorem 11.1], and we denote the element represented by $\mathcal{A}$ in the Brauer group $\operatorname{Br}(F)$ of $F$ by $\left[u_{1}, u_{2} ; m, F, \theta\right]$. 


\section{Lemma 11.2.2.}

(a) $Q(\theta) \simeq_{R_{2}}\left(t_{1}, t_{2} ; m, R_{2}, \theta\right)$

(b) $Q(\theta) \otimes_{R_{2}} K_{2} \simeq K_{2}\left(t_{1}, t_{2} ; m, K_{2}, \theta\right)$

(c) $Q(\theta) \otimes_{R_{2}} K_{2}$ is a central division algebra of dimension $m^{2}$ over $K_{2}$.

(d) $\left[t_{1}, t_{2} ; m, K_{2}, \theta\right]$ has order $m$ in $\operatorname{Br}\left(K_{2}\right)$.

Proof. (a) follows easily from the presentations for the two algebras, and (b) follows from (a).

(c) is well known (see for example the arguments in [Y1, §2-3]). For the reader's convenience, we briefly recall the proof. In view of (b) and the remarks in 11.2.1, it suffices to show that $Q(\theta) \otimes_{R_{2}} K_{2}$ is a division algebra. Since $Q(\theta) \otimes_{R_{2}} K_{2}$ is finite dimensional over $K_{2}$, it is enough to show that $Q(\theta) \otimes_{R_{2}} K_{2}$ is a domain, and thus it suffices to show that $Q(\theta)$ is a domain. This is easily seen looking at components of highest degree (relative to the lexicographic ordering) in the $\mathbb{Z}^{2}$-grading for $Q(\theta)$.

To prove (d), let $r$ be the order of $\left[t_{1}, t_{2} ; m, K_{2}, \theta\right]$ in $\operatorname{Br}\left(K_{2}\right)$. Since the algebra $\left(t_{1}, t_{2} ; m, K_{2}, \theta\right)$ is central simple of dimension $m^{2}$ over $K_{2}$, we know $r$ divides $m$. But by [D, Lemma 11.6], we have $r\left[t_{1}, t_{2} ; m, K_{2}, \theta\right]=\left[t_{1}, t_{2} ; \frac{m}{r}, K_{2}, \theta^{r}\right]$, so

$$
\left[t_{1}, t_{2} ; \frac{m}{r}, K_{2}, \theta^{r}\right]=0 \text {. }
$$

Also, by (b), $\left(t_{1}, t_{2} ; \frac{m}{r}, K_{2}, \theta^{r}\right) \simeq_{R_{2}} Q\left(\theta^{r}\right) \otimes_{R_{2}} K_{2}$, which is a division algebra of dimension $\left(\frac{m}{r}\right)^{2}$ over $K_{2}$ by (c). Hence, $\frac{m}{r}=1$, so $r=m$.

Remark 11.2.3. It is known that the homomorphism $\operatorname{Br}\left(R_{2}\right) \mapsto \operatorname{Br}\left(K_{2}\right)$ induced by base ring extension is an injection [Mi, Cor. IV.2.6], where $\operatorname{Br}\left(R_{2}\right)$ is the Brauer group of the ring $R_{2}$. Using this fact, Lemma 11.2.2(d) is equivalent to the statement, proved in [GP1, Prop. 3.16] in a different way, that $\left(t_{1}, t_{2} ; m, R_{2}, \theta\right)$ represents an element of order $m$ in $\operatorname{Br}\left(R_{2}\right)$.

\subsection{Isomorphism conditions.}

Lemma 11.3.1. Suppose that $\theta_{1}$ and $\theta_{2}$ are elements of $\mathbb{k}^{\times}$of finite order. If $Q\left(\theta_{1}\right) \otimes_{R_{2}} K_{2} \simeq_{K_{2}} Q\left(\theta_{2}\right) \otimes_{R_{2}} K_{2}$, then $\theta_{1}=\theta_{2}$.

Proof. By Lemma 11.2.2(c), $\theta_{1}$ and $\theta_{2}$ have the same order $m$ in $\mathbb{k}^{\times}$. So we can write $\theta_{2}=\theta_{1}^{q}$, where $q \in \mathbb{Z}$ and $\operatorname{gcd}(q, m)=1$. Then, by Lemma 11.2.2(b), we have $\left[t_{1}, t_{2} ; m, K_{2}, \theta_{1}\right]=\left[t_{1}, t_{2} ; m, K_{2}, \theta_{1}^{q}\right]$ in $\operatorname{Br}\left(K_{2}\right)$. So $q\left[t_{1}, t_{2} ; m, K_{2}, \theta_{1}\right]=$ $q\left[t_{1}, t_{2} ; m, K_{2}, \theta_{1}^{q}\right]$. But, by [D, Lemma 11.5], the right hand side is equal to $\left[t_{1}, t_{2} ; m, K_{2}, \theta_{1}\right]$. So $(q-1)\left[t_{1}, t_{2} ; m, K_{2}, \theta_{1}\right]=0$, and therefore, by Lemma 11.2.2(d), we have $q \equiv 1(\bmod m)$. Thus $\theta_{1}=\theta_{2}$.

Theorem 11.3.2. Suppose that $g_{1}$ and $g_{2}$ are positive integers, and that $\theta_{1}$ and $\theta_{2}$ are elements of $\mathbb{k}^{\times}$of finite order. Then, the following statements are equivalent:

(a) $\mathfrak{s l}_{g_{1}}\left(Q\left(\theta_{1}\right)\right) \simeq \mathfrak{s l}_{g_{2}}\left(Q\left(\theta_{2}\right)\right)$

(b) $g_{1}=g_{2}$ and $\theta_{1}=\theta_{2}^{ \pm 1}$.

(c) $\mathrm{M}_{g_{1}}\left(Q\left(\theta_{1}\right)\right) \simeq \mathrm{M}_{g_{2}}\left(Q\left(\theta_{2}\right)\right)$

Proof. For simplicity, we write $R=R_{2}$ and $K=K_{2}$.

"(c) $\Rightarrow(\mathrm{a})$ " is clear, and, since $Q\left(\theta_{1}\right) \simeq Q\left(\theta_{1}^{-1}\right)$, "(b) $\Rightarrow(\mathrm{c})$ " is also clear. So all that must be proved is that " $(\mathrm{a}) \Rightarrow(\mathrm{b})$ ".

Let $\mathcal{L}_{i}=\mathfrak{s l}_{g_{i}}\left(Q\left(\theta_{i}\right)\right)$ for $i=1,2$, and suppose that $\rho: \mathcal{L}_{1} \rightarrow \mathcal{L}_{2}$ is an isomorphism of $\mathbb{k}$-algebras. Let $m_{i}$ be the order of $\theta_{i}$ for $i=1,2$. If $g_{1} m_{1}=1$, then $g_{1}=1$ and $\theta_{1}=1$. Thus $\mathcal{L}_{1}=0$, so $\mathcal{L}_{2}=0$. Therefore, $g_{2}=1$ and $\theta_{2}=1$, so (b) 
holds. Hence, we can assume that $g_{1} m_{1}>1$ and similarly $g_{2} m_{2}>1$. Thus, by Lemma 4.5.6(e), the natural homomorphism $R \rightarrow C\left(\mathcal{L}_{i}\right)$ is an isomorphism which we regard as an identification for $i=1,2$. So we have the induced automorphism $\varphi=C(\rho)$ of $R$ satisfying $\rho(r x)=\varphi(r) \rho(x)$ for $r \in R, x \in \mathcal{L}_{1}$ (see 3.1.3). Then, by Lemma 11.1.4, there exists an isomorphism $\psi: Q\left(\theta_{1}\right) \rightarrow Q\left(\theta_{1}^{\varepsilon}\right)$, where $\varepsilon= \pm 1$, such that $\left.\psi\right|_{R}=\varphi^{-1}$. Further, by the last isomorphism in Lemma 11.1.3, we can assume that $\varepsilon=1$. Now $\psi \in \operatorname{Aut}\left(Q\left(\theta_{1}\right)\right)$ induces $\tilde{\psi} \in \mathrm{M}_{g_{1}}\left(Q\left(\theta_{1}\right)\right)$, which restricts to $\left.\tilde{\psi}\right|_{\mathcal{L}_{1}} \in \operatorname{Aut}\left(\mathcal{L}_{1}\right)$. Then, replacing $\rho$ by $\left.\rho \tilde{\psi}\right|_{\mathcal{L}_{1}}$, we can assume that $\rho$ is $R$-linear. Thus, $\mathcal{L}_{1} \simeq_{R} \mathcal{L}_{2}$, so $\mathcal{L}_{1} \otimes_{R} K \simeq_{K} \mathcal{L}_{2} \otimes_{R} K$.

Now since $K / R$ is a flat extension of commutative $k$-algebras [Bo1, Chap I, $\S 2.4$, Thm 1], we have $\left(\mathcal{M} \otimes_{R} K\right)^{\prime} \simeq_{K} \mathcal{M}^{\prime} \otimes_{R} K$ for any Lie algebra $\mathcal{M}$ over $R$. Thus,

$$
\begin{aligned}
\mathfrak{s l}_{g_{i}}\left(Q\left(\theta_{i}\right) \otimes_{R} K\right) & \simeq_{K}\left(\mathfrak{g l}_{g_{i}}\left(Q\left(\theta_{i}\right) \otimes_{R} K\right)\right)^{\prime} \simeq_{K}\left(\mathfrak{g l}_{g_{i}}\left(Q\left(\theta_{i}\right)\right) \otimes_{R} K\right)^{\prime} \\
& \simeq_{K}\left(\mathfrak{g l}_{g_{i}}\left(Q\left(\theta_{i}\right)\right)\right)^{\prime} \otimes_{R} K \simeq_{K} \mathfrak{s l}_{g_{i}}\left(Q\left(\theta_{i}\right)\right) \otimes_{R} K \simeq \mathcal{L}_{i} \otimes_{R} K
\end{aligned}
$$

for $i=1,2$. So

$$
\mathfrak{s l}_{g_{1}}\left(Q\left(\theta_{1}\right) \otimes_{R} K\right) \simeq_{K} \mathfrak{s l}_{g_{2}}\left(Q\left(\theta_{2}\right) \otimes_{R} K\right) .
$$

Now, by Lemma 11.2.2, $Q\left(\theta_{i}\right) \otimes_{R} K$ is an $m_{i}^{2}$-dimensional central division algebra over $K$ for $i=1,2$. Thus, by the isomorphism theorem for central simple Lie algebras of type $A$ [J, Thm. X.10], (31) implies that $\mathrm{M}_{g_{1}}\left(Q\left(\theta_{1}\right) \otimes_{R} K\right)$ is isomorphic as an algebra over $K$ to either $\mathrm{M}_{g_{2}}\left(Q\left(\theta_{2}\right) \otimes_{R} K\right)$ or $\left(\mathrm{M}_{g_{2}}\left(Q\left(\theta_{2}\right) \otimes_{R} K\right)\right)^{\mathrm{op}}$. Now, by Lemma 11.1.3, we can replace $\theta_{2}$ by $\theta_{2}^{-1}$ if necessary and assume that

$$
\mathrm{M}_{g_{1}}\left(Q\left(\theta_{1}\right) \otimes_{R} K\right) \simeq_{K} \mathrm{M}_{g_{2}}\left(Q\left(\theta_{2}\right) \otimes_{R} K\right)
$$

By the uniqueness part of Wedderburn's structure theorem [Pi, Thm. 3.5(ii)], it follows that $g_{1}=g_{2}$ and $Q\left(\theta_{1}\right) \otimes_{R} K \simeq_{K} Q\left(\theta_{2}\right) \otimes_{R} K$. Thus, $\theta_{1}=\theta_{2}$ by Lemma 4.1.9.

\section{Calculating the Relative type in the nontransitive Case}

Suppose that $\mathfrak{g}=\mathfrak{g}(A)$ is the Kac-Moody Lie algebra constructed from an affine $G C M A=\left(a_{i j}\right)_{i, j \in \mathrm{I}}$, where $\mathrm{I}=\{0, \ldots, \ell\}$ with $\ell \geq 1$, and that $\sigma \in \operatorname{Aut}(A)$ is not transitive. We continue with the notation of Section 7 for affine algebras, and we choose a positive integer $m$ such that $\sigma^{m}=1$.

We saw in Theorem 9.2.1 that the relative type of $\mathrm{L}\left(\overline{\mathfrak{g}^{\prime}}, \sigma\right)$ is the type of the finite root system $\overline{\pi_{\sigma}(\Delta)}$. In this section we use methods from [FSS] and [Bau] to compute the type of $\overline{\pi_{\sigma}(\Delta)}$.

\subsection{Calculating $\pi_{\sigma}(\Delta)^{\times}$.}

12.1.1. Recall from 9.1.2 that $\sigma$ acts on $\mathfrak{h}^{*}$ and that $\sigma(\Delta)=\Delta$, so $\sigma(V)=V$. Also, by (16), we have $\sigma\left(\alpha_{i}\right)=\alpha_{\sigma(i)}$ for $i \in \mathrm{I}$. Further, since $\sigma$ preserves the form $(\mid)$ on $\mathfrak{h}^{*}$ by Proposition 7.2.1, $\sigma$ also preserves the form $(\mid)$ on $V$.

Recall next that $V=V^{\sigma} \oplus(1-\sigma)(V)$. In fact, since $\sigma$ preserves the form ( | ) this decomposition is orthogonal:

$$
V=V^{\sigma} \perp(1-\sigma)(V) .
$$


As in 9.1.2, let $\pi_{\sigma}: V \rightarrow V^{\sigma}$ be the projection of $V$ onto $V^{\sigma}$ relative to the decomposition (32). Then, since $\sigma$ has period $m$, we have

$$
\pi_{\sigma}(\alpha)=\frac{1}{m} \sum_{i=0}^{m-1} \sigma^{i}(\alpha)
$$

for $\alpha \in V$.

12.1.2. Although we will not use this fact, it is clear that $\mathfrak{h}=\mathfrak{g}^{\sigma} \perp(1-\sigma) \mathfrak{h}$, $\left.\pi_{\sigma}(\alpha)\right|_{\mathfrak{h}^{\sigma}}=\left.\alpha\right|_{\mathfrak{h}^{\sigma}}$ and $\left.\pi_{\sigma}(\alpha)\right|_{(1-\sigma) \mathfrak{h}}=0$. In this way, $\pi_{\sigma}(\alpha)$ can be identified with $\left.\alpha\right|_{\mathfrak{h}^{\sigma}}$. This is the point of view taken in [Bau] and [ABP1].

12.1.3. Let

$$
\breve{\mathrm{I}}=\left\{i \in \mathrm{I} \mid \sigma^{k}(i) \geq i \text { for } k \in \mathbb{Z}\right\} .
$$

For $i \in \breve{\mathrm{I}}$, let $\mathcal{O}(i)$ denote the orbit containing $i$ under the action of the group $\langle\sigma\rangle$. Then the sets $\mathcal{O}(i), i \in \breve{\mathrm{I}}$, are the distinct orbits for the action of $\langle\sigma\rangle$ on $\mathrm{I}$; and, for $i \in \mathrm{I}, i$ is the least element of $\mathcal{O}(i)$.

For $i \in \breve{\mathrm{I}}$, set

$$
\mu_{i}=\pi_{\sigma}\left(\alpha_{i}\right) .
$$

Then $\pi_{\sigma}\left(\alpha_{p}\right)=\mu_{i}$ for $i \in \breve{\mathrm{I}}$ and $p \in \mathcal{O}(i)$. Also $\left\{\mu_{i}\right\}_{i \in \breve{\mathrm{I}}}$ is a $\mathbb{Q}$-basis for $V^{\sigma}$, so $\operatorname{dim}_{\mathbb{Q}}\left(V^{\sigma}\right)=\operatorname{card}(\breve{I})$.

12.1.4. Since $\sigma\left(\alpha_{i}\right)=\alpha_{\sigma(i)}$ for $i \in \mathrm{I}$, we have $\sigma(\delta)=\delta$ by (12) and (13). So $\delta \in V^{\sigma}$, and hence $\operatorname{dim}_{\mathbb{Q}}\left(\overline{V^{\sigma}}\right)=\operatorname{card}(\breve{\mathrm{I}})-1$.

The next lemma was observed in the proof of Proposition III.3.4 of [Bau] (see also [FSS, §2.5]). It is proved by checking the claim for each possible affine matrix $A$ and each $\sigma \in \operatorname{Aut}(A)$ that is not transitive.

Lemma 12.1.5. If $i \in \breve{\mathrm{I}}$, then exactly one of the following holds:

(a) The elements of $\mathcal{O}(i)$ are pairwise orthogonal.

(b) $\mathcal{O}(i)=\left\{\alpha_{p}, \alpha_{q}\right\}$, where $p, q \in \breve{\mathrm{I}}, p \neq q$ and $a_{p q}=a_{q p}=-1$.

12.1.6. For $i \in \breve{\mathrm{I}}$, we now define

$$
s_{i}= \begin{cases}1, & \text { if (a) holds in Lemma 12.1.5; } \\ 2, & \text { if (b) holds in Lemma 12.1.5. }\end{cases}
$$

Lemma 12.1.7. [FSS, §2.1] Let $i \in \breve{\mathrm{I}}$. Then $s_{i}\left(3-s_{i}\right)=2$ and

$$
s_{i}=3-\sum_{p \in \mathcal{O}(i)} a_{p i} .
$$

Proof. This follows immediately from Lemma 12.1.5 and the definition of $s_{i}$.

We now calculate the Cartan integers for the set $\left\{\mu_{i}\right\}_{i \in \breve{I}}$.

Proposition 12.1.8. Suppose that $i, j \in \breve{I}$. Then

(a) $\left(\mu_{i} \mid \mu_{i}\right) \neq 0$, so $\mu_{i} \in \pi_{\sigma}(\Delta)^{\times}$.

(b) If $\alpha \in V$, we have $\frac{\left(\mu_{i} \mid \pi_{\sigma}(\alpha)\right)}{\left(\mu_{i} \mid \mu_{i}\right)}=s_{i} \sum_{p \in \mathcal{O}(i)} \frac{\left(\alpha_{p} \mid \alpha\right)}{\left(\alpha_{p} \mid \alpha_{p}\right)}$.

(c) $2 \frac{\left(\mu_{i} \mid \mu_{j}\right)}{\left(\mu_{i} \mid \mu_{i}\right)}=s_{i} \sum_{p \in \mathcal{O}(i)} a_{p j} \in s_{i} \mathbb{Z}$. 
Proof. Suppose that $\alpha \in V$. Then

$$
\begin{aligned}
\left(\mu_{i} \mid \pi_{\sigma}(\alpha)\right) & =\left(\mu_{i} \mid \frac{1}{m} \sum_{p=0}^{m-1} \sigma^{p}(\alpha)\right)=\left(\mu_{i} \mid \alpha\right) \quad\left(\text { since } \mu_{i} \in V^{\sigma}\right) \\
& =\frac{1}{m}\left(\sum_{p=0}^{m-1} \sigma^{p}\left(\alpha_{i}\right) \mid \alpha\right)=\frac{1}{m} \frac{m}{\operatorname{card}(\mathcal{O}(i))}\left(\sum_{p \in \mathcal{O}(i)} \alpha_{p} \mid \alpha\right) \\
& =\frac{\left(\alpha_{i} \mid \alpha_{i}\right)}{\operatorname{card}(\mathcal{O}(i))} \sum_{p \in \mathcal{O}(i)} \frac{\left(\alpha_{p} \mid \alpha\right)}{\left(\alpha_{i} \mid \alpha_{i}\right)}=\frac{\left(\alpha_{i} \mid \alpha_{i}\right)}{\operatorname{card}(\mathcal{O}(i))} \sum_{p \in \mathcal{O}(i)} \frac{\left(\alpha_{p} \mid \alpha\right)}{\left(\alpha_{p} \mid \alpha_{p}\right)} .
\end{aligned}
$$

Now putting $\alpha=\alpha_{i}$ in (35), we get

$$
\left(\mu_{i} \mid \mu_{i}\right)=\frac{\left(\alpha_{i} \mid \alpha_{i}\right)}{2 \operatorname{card}(\mathcal{O}(i))} \sum_{p \in \mathcal{O}(i)} a_{p i}=\frac{\left(\alpha_{i} \mid \alpha_{i}\right)}{2 \operatorname{card}(\mathcal{O}(i))}\left(3-s_{i}\right)=\frac{\left(\alpha_{i} \mid \alpha_{i}\right)}{\operatorname{card}(\mathcal{O}(i))} \frac{1}{s_{i}} .
$$

using Lemma 12.1.7. This implies (a). Also, dividing (35) by (36), we obtain (b). Finally, setting $\alpha=\alpha_{j}$ in (b) yields (c).

12.1.9. Let $\breve{A}=\left(\breve{a}_{i j}\right)_{i, j \in \breve{\mathrm{I}}} \in \mathrm{M}_{\text {card }(\breve{\mathrm{I}})}(\mathbb{Q})$, where

$$
\breve{a}_{i j}=2 \frac{\left(\mu_{i} \mid \mu_{j}\right)}{\left(\mu_{i} \mid \mu_{i}\right)}
$$

for $i, j \in \breve{\mathrm{I}}$. By Proposition 12.1.8(c), we have

$$
\breve{a}_{i j}=s_{i} \sum_{p \in \mathcal{O}(i)} a_{p j} \in s_{i} \mathbb{Z}
$$

for $i, j \in \breve{\mathrm{I}}$, so in particular $\breve{A} \in \mathrm{M}_{\text {card }(\breve{\mathrm{I}})}(\mathbb{Z})$.

The next result is a special case of a result proved in [FSS] about diagram automorphisms of symmetrizable GCM's (see also [Bau, Prop.III.3.3]).

Proposition 12.1.10. $\breve{A}$ is an affine $G C M$.

Proof. In [FSS], $s_{i}$ and $\breve{a}_{i j}$ are defined by (34) and (38) respectively. With those definitions the proposition is proved in [FSS, §2.3].

Remark 12.1.11. It follows from (38) that the Dynkin diagram for $\breve{A}$ is obtained from the diagram for $A$ as follows. If $i, j \in \breve{\mathrm{I}}$,

Multiplicity of the arrow

from $\beta_{j}$ to $\beta_{i}$ in the $\quad=s_{i} \sum_{p \in \mathcal{O}(i)}$ including multiplicity, in the
diagram for $\breve{A}$

(If the sum on the right is zero, there is no arrow from $\beta_{j}$ to $\beta_{i}$.) See $\mathbf{1 2 . 2 . 2}$ for an example.

Remark 12.1.12. The type of the matrix $\breve{A}$ for each $A$ and each $\sigma$ (up to conjugacy in $\operatorname{Aut}(A))$ is recorded in [Bau, List II, $\S I I I .4]$ and in [FSS, Table 2.24].

12.1.13. If $\alpha \in V^{\times}$, we define the orthogonal reflection $r_{\alpha}: V \rightarrow V$ along $\alpha$ as usual as

$$
r_{\alpha}(\beta)=\beta-2 \frac{(\alpha \mid \beta)}{(\alpha \mid \alpha)} \alpha
$$

for $\beta \in V$. Then $r_{\alpha}^{2}=1$ and $r_{\alpha}$ is in the orthogonal group of the form $(\mid)$ on $V$. Let

$$
\breve{W}=\left\langle r_{\mu_{i}} \mid i \in \mathrm{I}\right\rangle \leq \mathrm{GL}(V) .
$$


Note that each $r_{\mu_{i}}$ fixes $(1-\sigma)(V)$ pointwise, so we can, by restriction, identify $\breve{W}$ with a subgroup of $\mathrm{GL}\left(V^{\sigma}\right)$.

12.1.14. Let $\breve{\Delta}$ be the set of roots (including 0) of the affine Kac-Moody Lie algebra $\mathfrak{g}(\breve{A})$, and let $\breve{V}=\operatorname{span}_{\mathbb{Q}}(\breve{\Delta})$. It follows from Proposition 12.1 .10 and (37) that we can identify $V^{\sigma}$ with $\breve{V}$ so that $\left\{\mu_{i}\right\}_{i \in \breve{I}}$ is the standard base for $\breve{\Delta}$ and the form ( | ) on $V^{\sigma}$ is a nonzero rational multiple of the standard form on $\breve{V}$. We make this identification from this point on. Then, $\breve{W}$ is the Weyl group of $\breve{\Delta}$. Hence, by (15) applied to $\mathfrak{g}(\breve{A})$, we have

$$
\breve{\Delta}^{\times}=\cup_{i \in \breve{\mathrm{I}}} \breve{W} \mu_{i} .
$$

12.1.15. In [Bau, pp. 33-37], J. Bausch proved the next proposition in the case when $\mathbb{k}=\mathbb{C}$ by constructing a subalgebra of $\mathfrak{g}^{\sigma}$ that is isomorphic to $\mathfrak{g}(\breve{A})$ and then using a characterization [K2, Prop. 5.8(a)] of the Tits cone of $\mathfrak{g}(\breve{A})$. The result for arbitrary $\mathbb{k}$ can likely be proved along the same lines. Instead, for the convenience of the reader, we present a proof by induction on the height of an element of $\pi_{\sigma}(\Delta)$.

Proposition 12.1.16. If $s_{i}=1$ for $i \in \breve{\mathrm{I}}$, then

$$
\pi_{\sigma}(\Delta)^{\times}=\breve{\Delta}^{\times} .
$$

Proof. We first show as in $[\mathrm{Bau}]$ that

$$
\breve{W}\left(\pi_{\sigma}(\Delta)\right) \subseteq \pi_{\sigma}(\Delta) .
$$

To prove this, we must show that $r_{\mu_{i}}\left(\pi_{\sigma}(\Delta)\right) \subseteq \pi_{\sigma}(\Delta)$ for $i \in \breve{\mathrm{I}}$. Indeed, since $s_{i}=1$, the refections $r_{\alpha_{p}}, p \in \mathcal{O}(i)$, commute. Set $w=\prod_{p \in \mathcal{O}(i)} r_{\alpha_{p}} \in W$. Then, for $\alpha \in \Delta$, we have, using Proposition 12.1.8(b),

$$
\begin{aligned}
r_{\mu_{i}}\left(\pi_{\sigma}(\alpha)\right) & =\pi_{\sigma}(\alpha)-2 \frac{\left(\mu_{i} \mid \pi_{\sigma}(\alpha)\right)}{\left(\mu_{i} \mid \mu_{i}\right)} \mu_{i}=\pi_{\sigma}(\alpha)-2\left(\sum_{p \in \mathcal{O}(i)} \frac{\left(\alpha_{p} \mid \alpha\right)}{\left(\alpha_{p} \mid \alpha_{p}\right)}\right) \mu_{i} \\
& =\pi_{\sigma}\left(\alpha-\sum_{p \in \mathcal{O}(i)} 2 \frac{\left(\alpha_{p} \mid \alpha\right)}{\left(\alpha_{p} \mid \alpha_{p}\right)} \alpha_{p}\right)=\pi_{\sigma}(w(\alpha)) \in \pi_{\sigma}(\Delta) .
\end{aligned}
$$

This proves (42); and, since reflections preserve the form $(\mid)$, it follows that

$$
\breve{W}\left(\pi_{\sigma}(\Delta)^{\times}\right) \subseteq \pi_{\sigma}(\Delta)^{\times} .
$$

The inclusion " $\supseteq$ " in (41) now follows from (40) and (43). For the inclusion "ᄃ $\subseteq$ ", observe first that any nonzero element $\nu$ of $\pi_{\sigma}(\Delta)$ can be written uniquely in the form $\nu=\sum_{i \in \breve{I}} n_{i} \mu_{i}$, where the $n_{i}$ are integers which are all either nonnegative or nonpositive. We say that $\nu$ is positive or negative accordingly, and we define the height of $\nu$ to be $\sum_{i \in \breve{I}} n_{i}$. We must show that

$$
\nu \in \pi_{\sigma}(\Delta)^{\times} \Longrightarrow \nu \in \breve{\Delta}^{\times} .
$$

To do this, we can assume that $\nu$ is positive and induct on the height of $\nu$, the case of height 1 being clear. Write $\nu=\sum_{i \in \breve{I}} n_{i} \mu_{i}$, where each $n_{i}$ is nonnegative. Then, since $(\nu \mid \nu)>0$, we have $\left(\nu \mid \mu_{i}\right)>0$ for some $i \in \breve{\mathrm{I}}$ with $n_{i}>0$. If $r_{\mu_{i}}(\nu)$ is positive, we're done by induction. So we can assume that $r_{\mu_{i}}(\nu)$ is negative. Then $\nu=q \mu_{i}$, where $q$ is a positive integer. Thus, we have $\nu=\pi_{\sigma}(\alpha)$, where $\alpha=\sum_{p \in \mathcal{O}(i)} m_{p} \alpha_{p} \in \Delta$ and $\sum_{p \in \mathcal{O}(i)} m_{p}=q$. Since $s_{i}=1$ for $i \in \breve{\mathrm{I}}$, this implies that $q=1$. 


\subsection{Calculating the relative type.}

Theorem 12.2.1. Suppose that $\mathfrak{g}=\mathfrak{g}(A)$ is an affine Kac-Moody Lie algebra and $\sigma \in \operatorname{Aut}(A)$ is not transitive. If $s_{i}=1$ for all $i \in \overline{\mathrm{I}}$ then the relative type of $\mathrm{L}\left(\overline{\mathfrak{g}^{\prime}}, \sigma\right)$ is equal to the quotient type of $\mathfrak{g}(\breve{A})$, where $\breve{A}$ is obtained from $A$ using (38); whereas if $s_{i}=2$ for some $i \in \breve{\mathrm{I}}$ then the relative type of $\mathrm{L}\left(\overline{\mathfrak{g}^{\prime}}, \sigma\right)$ is equal to $B C_{\text {card }(\breve{\mathrm{I}})-1}$. (Here the integers $s_{i}, i \in \breve{\mathrm{I}}$, are defined in 12.1.6.)

Proof. By Theorem 9.2.1, the relative type of $\mathrm{L}\left(\overline{\mathfrak{g}^{\prime}}, \sigma\right)$ is the type of the finite root system $\overline{\pi_{\sigma}(\Delta)}$.

If $s_{i}=1$ for all $i \in \breve{\mathrm{I}}$, then by (41) we have $\pi_{\sigma}(\Delta)^{\times}=\breve{\Delta}^{\times}$, and hence

$$
\overline{\pi_{\sigma}(\Delta)}=\overline{\pi_{\sigma}(\Delta)} \times\{0\}=\overline{\pi_{\sigma}(\Delta)^{\times}} \cup\{0\}=\overline{\breve{\Delta} \times} \cup\{0\}=\overline{\breve{\Delta}}^{\times} \cup\{0\}=\bar{\Delta} .
$$

Suppose finally that $s_{i}=2$ for some $i \in \breve{\mathrm{I}}$. Then, $\mathcal{O}(i)=\left\{\alpha_{p}, \alpha_{q}\right\}$, where $p, q \in$ $\breve{\mathrm{I}}, p \neq q$ and $a_{p q}=a_{q p}=-1$. So $\alpha_{p}+\alpha_{q} \in \Delta$, and hence $2 \mu_{i}=\pi_{\sigma}\left(\alpha_{p}+\alpha_{q}\right) \in \pi_{\sigma}(\Delta)$. Thus $\mu_{i}$ and $2 \mu_{i}$ are elements of $\pi_{\sigma}(\Delta)^{\times}$. So $\overline{\mu_{i}}$ and $2 \overline{\mu_{i}}$ are nonzero elements of $\overline{\pi_{\sigma}(\Delta)}$. Therefore $\overline{\pi_{\sigma}(\Delta)}$ is a nonreduced irreducible finite root system in $\overline{V^{\sigma}}$. Thus, by the classification of irreducible finite root systems [Bo2, Chap. VI, §4, no. 14], $\overline{\pi_{\sigma}(\Delta)}$ has type $\mathrm{BC}_{r}$, where $r=\operatorname{dim} \overline{V^{\sigma}}=\operatorname{card}(\breve{\mathrm{I}})-1$, using 12.1.4.

Example 12.2.2. In this example let $\mathfrak{g}=\mathfrak{g}(A)$, where $A$ has type $\mathrm{D}_{5}^{(1)}$ with Dynkin diagram

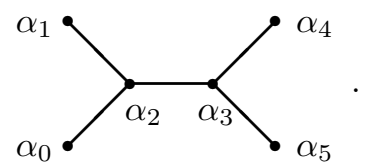

If $\sigma \in \operatorname{Aut}(A)$, we know from Corollary 6.3 .8 that the absolute type of $\mathrm{L}\left(\overline{\mathfrak{g}^{\prime}}, \sigma\right) \in \mathbb{M}_{2}$ is $\mathrm{D}_{5}$. We now use Theorem 12.2.1 to calculate the relative type of $\mathrm{L}\left(\overline{\mathfrak{g}^{\prime}}, \sigma\right)$ in two cases.

(a) Suppose first that $\sigma=(0,1)(4,5) \in \operatorname{Aut}(A)$. Then $\breve{I}=\{0,2,3,4\}$ and

$$
\mathcal{O}(0)=\{0,1\}, \quad \mathcal{O}(2)=\{2\}, \quad \mathcal{O}(3)=\{3\}, \quad \mathcal{O}(4)=\{4,5\} .
$$

Since $a_{01}=0$, we have $s_{0}=1$, and similarly $s_{2}=s_{3}=s_{4}=1$. We now use Remark 12.1.11 to calculate the Dynkin diagram for $\breve{A}$. We get

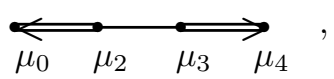

which is the diagram for the affine matrix of type $\mathrm{D}_{4}^{(2)}$. So, by Table 1 , the quotient type of $\mathfrak{g}(\breve{A})$ is $\mathrm{B}_{3}$; hence, by Theorem $12.2 .1, \mathrm{~L}\left(\overline{\mathfrak{g}^{\prime}}, \sigma\right)$ has relative type $\mathrm{B}_{3}$.

(b) Suppose next that $\sigma=(0,5)(1,4)(2,3) \in \operatorname{Aut}(A)$. Then $\breve{\mathrm{I}}=\{0,1,2\}$, and

$$
\mathcal{O}(0)=\{0,5\}, \quad \mathcal{O}(1)=\{1,4\}, \quad \mathcal{O}(2)=\{2,3\} .
$$

Since $a_{23}=a_{32}=-1$, we have $s_{2}=2$. Thus, by Theorem 12.2.1, $\mathrm{L}\left(\overline{\mathfrak{g}^{\prime}}, \sigma\right)$ has relative type $\mathrm{BC}_{2}$.

\section{Classification of algebras in $\mathbb{M}_{2}$}

In this section, we obtain a classification of the algebras in $\mathbb{M}_{2}$ up to isomorphism. 


\subsection{Tables of relative types.}

13.1.1. If $A$ is an affine GCM and $\sigma \in \operatorname{Aut}(A)$, the relative type of $\mathrm{L}\left(\overline{\mathfrak{g}(A)^{\prime}}, \sigma\right)$ can be calculated using the method described in Example 12.2.2 when $\sigma$ is not transitive and using Corollary 8.3.2 when $\sigma$ is transitive. In Tables 2 and 3 below, we record this relative type for all $A$ up to isomorphism and all $\sigma \in \operatorname{Aut}(A)$ up to conjugacy. Table 2 covers the cases when $\mathfrak{g}(A)$ is untwisted, whereas Table 3 covers the twisted cases. Our enumeration of the fundamental roots of the root system corresponding to $A$ follows [K2, $§ 4.8]$.

The first column of the tables contains the type $\mathrm{X}_{k}^{(m)}$ of $A$; and the second column contains $\sigma$ (when $\sigma$ is uniquely determined up to conjugacy in $\operatorname{Aut}(A)$ by its order, we simply list its order). The third column contains a label that we assign to the algebra $\mathrm{L}\left(\overline{\mathfrak{g}(A)^{\prime}}, \sigma\right)$ for ease of reference; and the fourth column contains the relative type of $\mathrm{L}\left(\overline{\mathfrak{g}(A)^{\prime}}, \sigma\right)$. When the entry in column 4 depends on the parity of $k$, the first expression in the column applies when $k$ is even and the second when $k$ is odd.

The labels in column 3 were chosen to indicate the method of construction of the algebras as iterated loop algebras. For example, we used the labels $\mathrm{D}_{k}^{(1,2 a)}, \mathrm{D}_{k}^{(1,2 b)}$ and $\mathrm{D}_{k}^{(1,2 c)}$ in lines 9,10 and 11 of Table 2 , since these algebras are constructed starting from a finite dimensional Lie algebra of type $\mathrm{D}_{k}$ using a loop construction relative to an automorphism of order 1 followed by a loop construction relative to an automorphism of order 2. The one exception to this scheme is the label $\mathrm{A}_{k}^{(1, \operatorname{rot}(q))}$ in Line 1 of Table 2, where we needed to convey more information with the label.

If $A$ and $\sigma$ are chosen from columns 1 and 2 of Tables 2 or 3, we will often denote the algebra $\mathrm{L}\left(\overline{\mathfrak{g}(A)^{\prime}}, \sigma\right)$ by the corresponding label from column 3.

Remark 13.1.2. It is important to recall that the absolute type of $\mathrm{L}\left(\overline{\mathfrak{g}(A)^{\prime}}, \sigma\right)$ can also be read from Tables 2 and 3. Indeed if $A$ has type $\mathrm{X}_{k}^{(m)}$, then $\mathrm{L}\left(\overline{\mathfrak{g}(A)^{\prime}}, \sigma\right)$ has absolute type $\mathrm{X}_{k}$ by Corollary 6.3.8.

13.1.3. Note that by (24) the algebras occurring in row 1 of Table 2 have matrix realizations:

$$
\mathrm{A}_{k}^{(1, \operatorname{rot}(q))} \simeq \mathfrak{s l}_{\operatorname{gcd}(q, k+1)}\left(Q\left(\zeta_{k+1}^{\iota_{k+1}(\bar{q})}\right)\right)
$$

$0 \leq q \leq\left\lfloor\frac{k+1}{2}\right\rfloor$. (Recall that if $q=0, \operatorname{gcd}(q, k+1)$ is interpreted as $k+1$.)

The following proposition follows from Theorem 10.1.1 and Table 2.

Proposition 13.1.4. Let $\mathcal{L} \in \mathbb{M}_{2}$. Then $\mathcal{L}$ satisfies condition (AA) (described in the introduction) if and only if $\mathcal{L} \simeq A_{k}^{(1, \operatorname{rot}(q))}$ for some $k \geq 1$ and some $0 \leq q \leq$ $\left\lfloor\frac{k+1}{2}\right\rfloor$.

\subsection{Isomorphism.}

We know from Theorem 10.1.1 that the algebras in $\mathbb{M}_{2}$ are, up to isomorphism, the algebras of the form $\mathrm{L}\left(\overline{\mathfrak{g}^{\prime}}, \sigma\right)$, where $\mathfrak{g}$ is an untwisted affine algebra and $\sigma$ is a diagram automorphism of $\overline{\mathfrak{g}^{\prime}}$. The next theorem give necessary and sufficient conditions for two algebras of this form to be isomorphic.

\section{Theorem 13.2.1.}

(a) If $\mathfrak{g}_{i}$ is an untwisted affine Kac-Moody Lie algebra and $\sigma_{i}$ is a diagram automorphism of $\overline{\mathfrak{g}_{i}^{\prime}}, i=1,2$, then $\mathrm{L}\left(\overline{\mathfrak{g}_{1}^{\prime}}, \sigma_{1}\right) \simeq \mathrm{L}\left(\overline{\mathfrak{g}_{2}^{\prime}}, \sigma_{2}\right)$ implies that $\mathfrak{g}_{1} \simeq \mathfrak{g}_{2}$. 


\begin{tabular}{|c|c|c|c|}
\hline$A$ & $\sigma$ & Label & Relative type \\
\hline \multirow[t]{3}{*}{$\mathrm{A}_{k}^{(1)}, k \geq 1$} & $(0,1, \ldots, k)^{q}, 0 \leq q \leq\left\lfloor\frac{k+1}{2}\right\rfloor$ & $\mathrm{A}_{k}^{(1, \operatorname{rot}(q))}$ & $\mathrm{A}_{\operatorname{gcd}(q, k+1)-1}$ \\
\hline & $\Pi_{i=1}^{\left\lfloor\frac{k}{2}\right\rfloor}(i, k+1-i), k \geq 2$ & $\mathrm{~A}_{k}^{(1,2 a)}$ & $\mathrm{BC}_{\frac{k}{2}}, \mathrm{C}_{\frac{k+1}{2}}$ \\
\hline & $\Pi_{i=0}^{\frac{k-1}{2}}(i, k-i), k$ odd $\geq 3$ & $\mathrm{~A}_{k}^{(1,2 b)}$ & $\mathrm{BC}_{\frac{k-1}{2}}$ \\
\hline \multirow[t]{2}{*}{$\mathrm{B}_{k}^{(1)}, k \geq 3$} & (1) & $\mathrm{B}_{k}^{(1,1)}$ & $\mathrm{B}_{k}$ \\
\hline & $|\sigma|=2$ & $\mathrm{~B}_{k}^{(1,2)}$ & $\mathrm{B}_{k-1}$ \\
\hline \multirow[t]{2}{*}{$\mathrm{C}_{k}^{(1)}, k \geq 2$} & $(1)$ & $\mathrm{C}_{k}^{(1,1)}$ & $\mathrm{C}_{k}$ \\
\hline & $|\sigma|=2$ & $\mathrm{C}_{k}^{(1,2)}$ & $\mathrm{C}_{\frac{k}{2}}, \quad \mathrm{BC}_{\frac{k-1}{2}}$ \\
\hline \multirow[t]{6}{*}{$\mathrm{D}_{k}^{(1)}, k \geq 4$} & (1) & $\mathrm{D}_{k}^{(1,1)}$ & $\mathrm{D}_{k}$ \\
\hline & $(k-1, k)$ & $\mathrm{D}_{k}^{(1,2 a)}$ & $\mathrm{B}_{k-1}$ \\
\hline & $(0,1)(k-1, k)$ & $\mathrm{D}_{k}^{(1,2 b)}$ & $\mathrm{B}_{k-2}$ \\
\hline & $\Pi_{i=0}^{\left\lfloor\frac{k-1}{2}\right\rfloor}(i, k-i), k \geq 5$ & $\mathrm{D}_{k}^{(1,2 c)}$ & $\mathrm{C}_{\frac{k}{2}}, \mathrm{BC}_{\frac{k-1}{2}}$ \\
\hline & $|\sigma|=3, k=4$ & $\mathrm{D}_{4}^{(1,3)}$ & $\mathrm{G}_{2}$ \\
\hline & $|\sigma|=4$ & $\mathrm{D}_{k}^{(1,4)}$ & $\mathrm{BC}_{\left\lfloor\frac{k-2}{2}\right\rfloor}$ \\
\hline \multirow[t]{3}{*}{$\overline{\mathrm{E}_{6}^{(1)}}$} & (1) & $\mathrm{E}_{6}^{(1,1)}$ & $\mathrm{E}_{6}$ \\
\hline & $|\sigma|=2$ & $\mathrm{E}_{6}^{(1,2)}$ & $\mathrm{F}_{4}$ \\
\hline & $|\sigma|=3$ & $\mathrm{E}_{6}^{(1,3)}$ & $\mathrm{G}_{2}$ \\
\hline \multirow[t]{2}{*}{$\overline{\mathrm{E}_{7}^{(1)}}$} & (1) & $\mathrm{E}_{7}^{(1,1)}$ & $\mathrm{E}_{7}$ \\
\hline & $|\sigma|=2$ & $\mathrm{E}_{7}^{(1,2)}$ & $\mathrm{F}_{4}$ \\
\hline $\mathrm{E}_{8}^{(1)}$ & (1) & $\mathrm{E}_{8}^{(1,1)}$ & $E_{8}$ \\
\hline $\mathrm{F}_{4}^{(1)}$ & (1) & $\mathrm{F}_{4}^{(1,1)}$ & $\mathrm{F}_{4}$ \\
\hline $\mathrm{G}_{2}^{(1)}$ & $(1)$ & $\mathrm{G}_{2}^{(1,1)}$ & $\mathrm{G}_{2}$ \\
\hline
\end{tabular}

TABle 2. Relative type of $\mathrm{L}\left(\overline{\mathfrak{g}(A)^{\prime}}, \sigma\right)$ for untwisted $A$

\begin{tabular}{|c|c|c|c|}
\hline$A$ & $\sigma$ & Label & Relative type \\
\hline \multirow{2}{*}{$\mathrm{A}_{k}^{(2)}, k \geq 2, k \neq 3$} & $(1)$ & $\mathrm{A}_{k}^{(2,1)}$ & $\mathrm{BC}_{\frac{k}{2}}, \mathrm{C}_{\frac{k+1}{2}}$ \\
\cline { 2 - 4 } & $|\sigma|=2, k$ odd & $\mathrm{A}_{k}^{(2,2)}$ & $\mathrm{BC}_{\frac{k-1}{2}}$ \\
\hline \multirow{2}{*}{$\mathrm{D}_{k}^{(2)}, k \geq 3$} & $(1)$ & $\mathrm{D}_{k}^{(2,1)}$ & $\mathrm{B}_{k-1}$ \\
\cline { 2 - 4 } & $\Pi_{i=0}^{\left\lfloor\frac{k-2}{2}\right\rfloor}(i, k-1-i)$ & $\mathrm{D}_{k}^{(2,2)}$ & $\mathrm{BC}_{\left\lfloor\frac{k-1}{2}\right\rfloor}$ \\
\hline $\mathrm{D}_{4}^{(3)}$ & $(1)$ & $\mathrm{D}_{4}^{(3,1)}$ & $\mathrm{G}_{2}$ \\
\hline $\mathrm{E}_{6}^{(2)}$ & $(1)$ & $\mathrm{E}_{6}^{(2,1)}$ & $\mathrm{F}_{4}$ \\
\hline
\end{tabular}

TABLE 3. Relative type of $\mathrm{L}\left(\overline{\mathfrak{g}(A)^{\prime}}, \sigma\right)$ for twisted $A$ 
(b) If $\mathfrak{g}$ is an affine Kac-Moody Lie algebra (twisted or untwisted) and $\sigma_{i}$ is a diagram automorphism of $\overline{\mathfrak{g}^{\prime}}, i=1,2$, then $\mathrm{L}\left(\overline{\mathfrak{g}^{\prime}}, \sigma_{1}\right) \simeq \mathrm{L}\left(\overline{\mathfrak{g}^{\prime}}, \sigma_{2}\right)$ if and only if $\sigma_{1}$ and $\sigma_{2}$ are conjugate in the group of diagram automorphisms of $\overline{\mathfrak{g}^{\prime}}$.

Proof. (a) follows from Corollary 6.3.8, so it remains to prove (b). The implication " $\Leftarrow$ " in (b) follows from Lemma 4.1.2(a).

To prove the implication " $\Rightarrow$ " in (b), suppose that $\mathcal{L}_{1} \simeq \mathcal{L}_{2}$, where $\mathcal{L}_{i}=\mathrm{L}\left(\overline{\mathfrak{g}^{\prime}}, \sigma_{i}\right)$ for $i=1,2$. Now $\mathfrak{g}=\mathfrak{g}(A)$, where $A$ is one of the affine matrices in column 1 of Tables 2 or 3. Further, replacing each $\sigma_{i}$ by a conjugate in $\operatorname{Aut}(A)$, we can, by Lemma 4.1.2(a), assume that $\sigma_{1}$ and $\sigma_{2}$ are among the automorphisms listed in column 2 of Tables 2 or 3 .

Case 1: Suppose that $\mathcal{L}_{1}$, and hence also $\mathcal{L}_{2}$, satisfies Condition (AA). Then by Proposition 13.1.4, $A$ has type $\mathrm{A}_{k}^{(1)}$ for some $k \geq 1$ and $\sigma_{i}=(0,1, \ldots, k)^{q_{i}}$, where $0 \leq q_{i} \leq\left\lfloor\frac{k+1}{2}\right\rfloor, i=1,2$. So $\mathcal{L}_{i}=\mathrm{L}\left(\overline{\mathfrak{g}^{\prime}},(0,1, \ldots, k)^{q_{i}}\right)$ for $i=1,2$. Thus, since $\mathcal{L}_{1} \simeq \mathcal{L}_{2}$, we have

$$
\mathfrak{s l}_{\operatorname{gcd}\left(q_{1}, k+1\right)}\left(Q\left(\zeta_{k+1}^{\iota_{k+1}\left(\bar{q}_{1}\right)}\right)\right) \simeq \mathfrak{s l}_{\operatorname{gcd}\left(q_{2}, k+1\right)}\left(Q\left(\zeta_{k+1}^{\iota_{k+1}\left(\bar{q}_{2}\right)}\right)\right)
$$

by (24). Hence, by Theorem 11.3.2, we have $\iota_{k+1}\left(\bar{q}_{1}\right)= \pm \iota_{k+1}\left(\bar{q}_{2}\right)$ in $\mathbb{Z}_{k+1}$, and therefore $\bar{q}_{1}= \pm \bar{q}_{2}$. So $q_{1}=q_{2}$, and $\sigma_{1}=\sigma_{2}$.

Case 2: Suppose that $\mathcal{L}_{1}$, and hence also $\mathcal{L}_{2}$, does not satisfy Condition (AA). If $\mathfrak{g}$ is untwisted, then $\sigma_{1}$ and $\sigma_{2}$ appear in one of the rows after row 1 of Table 2. Since the relative type of $\mathcal{L}_{1}$ is the same as the relative type of $\mathcal{L}_{2}$, it follows checking Table 2 that $\sigma_{1}$ and $\sigma_{2}$ appear in the same row. Thus, $\sigma_{1}=\sigma_{2}$. The argument in the twisted case is the same using Table 3 .

13.2.2. As a corollary of this theorem, we next prove that the converse in Theorem $6.2 .2(\mathrm{c})$ is valid for an affine algebra $\mathfrak{g}=\mathfrak{g}(A)$ and automorphisms of first kind. Before doing so, we note that by the classification of affine matrices, $\operatorname{Aut}(A)$ is one of the following: a cyclic group of order 1 or 2 , a dihedral group of order $\geq 6$, or a symmetric group $S_{3}$ or $S_{4}$. Hence, any element of $\operatorname{Aut}(A)$ is conjugate to its inverse, so the relation $\sim \operatorname{in} \operatorname{Aut}(A)$ described prior to Theorem 6.2.2 is simply conjugacy.

Corollary 13.2.3. Suppose that $\mathfrak{g}=\mathfrak{g}(A)$ is an affine Kac-Moody Lie algebra and $\sigma_{1}$ and $\sigma_{2}$ are finite order automorphisms of first kind of $\overline{\mathfrak{g}^{\prime}}$. Then $\mathrm{L}\left(\overline{\mathfrak{g}^{\prime}}, \sigma_{1}\right) \simeq$ $\mathrm{L}\left(\overline{\mathfrak{g}^{\prime}}, \sigma_{2}\right)$ if and only if $\bar{p}\left(\sigma_{1}\right)$ is conjugate to $\bar{p}\left(\sigma_{2}\right)$ in $\operatorname{Aut}(A)$.

Proof. By Theorem 6.2.2(c), we only need to prove the implication " $\Rightarrow$ ". Suppose that $\mathrm{L}\left(\overline{\mathfrak{g}^{\prime}}, \sigma_{1}\right) \simeq \mathrm{L}\left(\overline{\mathfrak{g}^{\prime}}, \sigma_{2}\right)$. By Theorem 6.2.2(c), we have $\mathrm{L}\left(\overline{\mathfrak{g}^{\prime}}, \sigma_{i}\right) \simeq \mathrm{L}\left(\overline{\mathfrak{g}^{\prime}}, \bar{p}\left(\sigma_{i}\right)\right)$, for $i=1,2$. Hence, replacing $\sigma_{i}$ by $\bar{p}\left(\sigma_{i}\right)$, we can assume that $\sigma_{i} \in \operatorname{Aut}(A), i=1,2$. The result now follows from Theorem 13.2.1(b).

\subsection{Classification.}

Our second main theorem gives a classification of the algebras in $\mathbb{M}_{2}$.

Theorem 13.3.1. If $A$ and $\sigma$ are chosen from Columns 1 and 2 of Table 2, then $\mathrm{L}\left(\overline{\mathfrak{g}(A)^{\prime}}, \sigma\right)$ is in $\mathbb{M}_{2}$. Conversely, any algebra in $\mathbb{M}_{2}$ is isomorphic to exactly one such algebra.

Proof. This follows from Theorems 10.1.1 and Theorems 13.2.1.

13.3.2. Using the labels in column 3 of Table 2, Theorem 13.3.1 states that a nonredundant list of all algebras in $\mathbb{M}_{2}$ up to isomorphism is $A_{k}^{(1, \operatorname{rot}(q))}, \ldots, \mathrm{G}_{2}^{(1,1)}$. 
The following corollary follows from Proposition 13.1.4, Theorem 13.3.1 and Table 2.

Corollary 13.3.3. Two algebras in $\mathbb{M}_{2}$ that do not satisfy Condition (AA) are isomorphic if and only if they have the same absolute type and the same relative type.

13.3.4. Any algebra appearing in Table 3 is in $\mathbb{M}_{2}$ by Theorem 10.1.1, and hence it is isomorphic to an algebra in Table 2. But any such algebra does not satisfy Condition (AA) (by Table 3), so to match it with an algebra in Table 2, we just have to match the relative and absolute types. In this way, we see that

$$
\begin{gathered}
\mathrm{A}_{k}^{(2,1)} \simeq \mathrm{A}_{k}^{(1,2 a)} \quad \text { if } k \geq 2, k \neq 3, \\
\mathrm{~A}_{k}^{(2,2)} \simeq \mathrm{A}_{k}^{(1,2 b)} \quad \text { if } k \geq 5, k \text { odd, } \\
\mathrm{D}_{k}^{(2,1)} \simeq \begin{cases}\mathrm{D}_{k}^{(1,2 a)}, & \text { if } k \geq 4 ; \\
\mathrm{A}_{3}^{(1,2 a)}, & \text { if } k=3,\end{cases} \\
\mathrm{D}_{k}^{(2,2)} \simeq \begin{cases}\mathrm{D}_{k}^{(1,4)}, & \text { if } k \geq 4, k \text { even; } \\
\mathrm{D}_{k}^{(1,2 c)}, & \text { if } k \geq 5, k \text { odd; } \\
\mathrm{A}_{3}^{(1,2 b)}, & \text { if } k=3, \\
\mathrm{D}_{4}^{(3,1)} \simeq \mathrm{D}_{4}^{(1,3)} \quad \text { and } \quad \mathrm{E}_{6}^{(2,1)} \simeq \mathrm{E}_{6}^{(1,2)} .\end{cases}
\end{gathered}
$$

The isomorphisms $\mathrm{A}_{k}^{(2,1)} \simeq \mathrm{A}_{k}^{(1,2 a)}, \mathrm{D}_{k}^{(2,1)} \simeq \mathrm{D}_{k}^{(1,2 a)}, \mathrm{D}_{4}^{(3,1)} \simeq \mathrm{D}_{4}^{(1,3)}$ and $\mathrm{E}_{6}^{(2,1)} \simeq$ $\mathrm{E}_{6}^{(1,2)}$ are of course not surprising and can be proved directly without our classification results.

\section{LINKS TO SOME RELATED WORK.}

In this section, we consider two invariants associated to an algebra $\mathcal{L}$ in $\mathbb{M}_{2}$, the index and the Saito extended affine root system, thereby linking our results to the work of several other researchers.

\subsection{The index of a prime perfect fgc Lie algebra.}

In order to define the index of a prime perfect fgc Lie algebra, we first discuss the corresponding notion for algebraic groups.

14.1.1. Suppose that $F$ is a field, $F_{s}$ is a separable closure of $F$, and $\Gamma=$ $\operatorname{Gal}\left(F_{s} / F\right)$.

(a) Starting from a semisimple algebraic group $G$ defined over $F$, one can construct a triple $\left(\Pi, \Pi_{0}, *\right)$, called the index of $G[\mathrm{~T}, \S 2.3]$. This triple, which is often also called the Tits index of $G$, consists of a finite Dynkin diagram $\Pi$, a (possibly empty) subset $\Pi_{0}$ of $\Pi$, and a homomorphism $\sigma \stackrel{*}{\rightarrow} \sigma^{*}$ (called the $*$-action) from $\Gamma$ into Aut( $\Pi)$ satisfying $\sigma^{*}\left(\Pi_{0}\right)=\Pi_{0}$ for $\sigma \in \Gamma$. (Here Aut( $\left.\Pi\right)$ is the group of diagram automorphisms of $\Pi$.) We will not need to describe the construction of $\left(\Pi, \Pi_{0}, *\right)$, but instead recall it below in $\mathbf{1 4 . 1 . 3}(\mathrm{b})$ in the special case of interest here.

(b) If $G_{i}$ is a semisimple algebraic group defined over $F$ with index $\left(\Pi_{i}, \Pi_{i 0}, *\right)$ for $i=1,2$, we say that $\left(\Pi_{1}, \Pi_{10}, *\right)$ and $\left(\Pi_{2}, \Pi_{20}, *\right)$ are isomorphic if there is a diagram isomorphism from $\Pi_{1}$ onto $\Pi_{2}$ which maps $\Pi_{10}$ onto $\Pi_{20}$ and commutes with the $*$-actions. As a special case of [T, Prop. 2.6.3], we know that if $G_{1}$ and 
$G_{2}$ are $F$-isomorphic then their indices are isomorphic. In particular, the index is independent up to isomorphism of the choices made in its construction.

14.1.2. Besides isomorphism, there is a another notion of equivalence for indices which is useful. To describe this, suppose that for $i=1,2, F_{i}$ is a field, $\left(F_{i}\right)_{s}$ is a separable closure of $F_{i}, \Gamma_{i}=\operatorname{Gal}\left(\left(F_{i}\right)_{s} / F_{i}\right)$, and $\left(\Pi_{i}, \Pi_{i 0}, *\right)$ is the index for a semisimple algebraic group $G_{i}$ defined over $F_{i}$. We say that the indices $\left(\Pi_{1}, \Pi_{10}, *\right)$ and $\left(\Pi_{2}, \Pi_{20}, *\right)$ are similar if there is a diagram isomorphism $\lambda: \Pi_{1} \rightarrow \Pi_{2}$ and a group isomorphism $\mu:\left(\Gamma_{1}\right)^{*} \rightarrow\left(\Gamma_{2}\right)^{*}$ such that $\lambda\left(\Pi_{10}\right)=\Pi_{20}$ and $\lambda(g \alpha)=$ $\mu(g) \lambda(\alpha)$ for $g \in\left(\Gamma_{1}\right)^{*}$ and $\alpha \in \Pi$. Clearly, if $F_{1}=F_{2}$, isomorphic indices are similar.

In the famous Table 2 of [T], Tits listed and labelled all indices up to similarity that can occur for some absolutely simple algebraic group defined over some $F$. We will use those labels ${ }^{1} \mathrm{~A}_{n, r}^{d}, \ldots, \mathrm{G}_{2,2}^{0}$ in Section 14.2 below.

14.1.3. Suppose next that $\mathcal{L}$ is a simple fgc Lie algebra. Let $F=\mathrm{C}(\mathcal{L})$, which is a field extension of $\mathbb{k}$.

(a) Recall from 3.1.2 that $\mathcal{L}$ is a finite dimensional central simple Lie algebra over $F$, and hence $\mathbf{A u t}^{0}(\mathcal{L})$ is a simple algebraic group over $F$, where $\mathbf{A u t}^{0}(\mathcal{L})$ denotes the connected component of the automorphism group $\operatorname{Aut}(\mathcal{L})$ of $\mathcal{L}$ over $F$. We define the index of $\mathcal{L}$ to be the index of $\operatorname{Aut}^{0}(\mathcal{L})$.

(b) Since $\operatorname{char}(\mathbb{k})=0$, the index of $\mathcal{L}$ can equivalently be defined purely in terms of the structure of $\mathcal{L}$. For the convenience of the reader, we do this here following $[\mathrm{T}, \S 2.3]$, [Sat, $\S 4]$ and [Se1] (in the Lie algebra case). Let $\bar{F}\left(=F_{s}\right)$ be an algebraic closure of $F$ with $\Gamma=\operatorname{Gal}(\bar{F} / F)$, let $\mathcal{L}_{\bar{F}}=\mathcal{L} \otimes_{F} \bar{F}$, and identify $\mathcal{L}$ as usual as an $F$-subalgebra of $\mathcal{L}_{\bar{F}}$. Let $\mathcal{T}$ be a MAD $F$-subalgebra of $\mathcal{L}$, and let $\mathcal{H}$ be a Cartan $F$-subalgebra of $\mathcal{L}$ containing $\mathcal{T}$ [Se2, Prop. I.1]. Then $\mathcal{H}_{\bar{F}}=\mathcal{H} \otimes_{F} \bar{F}$ is a MAD $\bar{F}$-subalgebra of $\mathcal{L}_{\bar{F}}$. Let $\Delta$ be the root system (including 0 ) of $\mathcal{L}_{\bar{F}}$ relative to $\mathcal{H}_{\bar{F}}$, in which case the restriction map $\alpha \mapsto \alpha_{\mathcal{J}}$ maps $\Delta$ onto the root system of $\mathcal{L}$ with respect to $\mathcal{T}$. Choose an additive linear order $>$ on the root lattice $Q(\Delta)$ that is compatible with restriction (that is if $\alpha_{1}, \alpha_{2} \in Q(\Delta),\left.\alpha_{1}\right|_{\mathcal{T}}=\left.\alpha_{2}\right|_{\mathcal{T}}$ and $\alpha_{1}>0$ then $\left.\alpha_{2}>0\right)$. Let $\Pi$ be the base for $\Delta$ determined by $>$, and let $\Pi_{0}=\left\{\alpha \in \Pi:\left.\alpha\right|_{\mathcal{T}}=0\right\}$. Finally, $\Gamma$ acts on $\mathcal{H}_{\bar{F}}$ (trivially on $\mathcal{H}$ and naturally on $\bar{F}$ ), and this action transfers as usual to an action of $\Gamma$ on $\mathcal{H}_{\bar{F}}^{*}=\operatorname{Hom}_{\bar{F}}\left(\mathcal{H}_{\bar{F}}, \bar{F}\right)$ which stabilizes $\Delta$. For $\sigma \in \Gamma$, we define $\sigma^{*} \in \operatorname{Aut}(\Pi)$, by $\sigma^{*}(\alpha)=w_{\sigma} \sigma(\alpha)$, where $w_{\sigma}$ is the element of the Weyl group of $\Delta$ such that $w_{\sigma} \sigma(\Pi)=\Pi$. Then $\left(\Pi, \Pi_{0}, *\right)$ is the index of $\mathcal{L}$

(c) If follows from the definition in (b) (or (a)) that the absolute type of $\mathcal{L}$ is the type of the Dynkin diagram $\Pi$. (This type is used as the "base" for the label of the index of $\mathcal{L}$.) Moreover, Tits described in $[\mathrm{T}, \S 2.5 .2]$ an algorithm for calculating the relative type of $\mathcal{L}$ given its index up to similarity. Hence, both the absolute and relative type of $\mathcal{L}$ are determined by its index up to similarity. For example if $\mathcal{L}$ has index $\mathrm{E}_{7,4}^{9}$, then $\mathcal{L}$ has absolute type $\mathrm{E}_{7}$ and, using the algorithm, relative type $\mathrm{F}_{4}$.

Definition 14.1.4. Suppose finally that $\mathcal{L}$ is an prime perfect fgc Lie algebra. We know from Proposition 3.3.2 that the central closure $\widetilde{\mathcal{L}}:=\mathcal{L} \otimes_{\mathrm{C}(\mathcal{L})} \widetilde{C}(\mathcal{L})$ is a simple fgc Lie algebra. We define the index of $\mathcal{L}$ to be the index of $\widetilde{\mathcal{L}}$.

Lemma 14.1.5. Suppose that $\mathcal{L}_{1}$ and $\mathcal{L}_{2}$ are prime perfect fgc Lie algebras that are isomorphic (as $\mathbb{k}$-algebras). Then the indices of $\mathcal{L}_{1}$ and $\mathcal{L}_{2}$ are similar. 
Proof. We sketch this proof; the details are straightforward and left to the reader. As in the proof of Lemma 3.3.4, we can assume that $\mathcal{L}_{1}$ and $\mathcal{L}_{2}$ are simple. Choose $F_{i}, \bar{F}_{i}$ and $\Gamma_{i}$ for $\mathcal{L}_{i}$ as in 14.1.3(b). Let $\varphi: \mathcal{L}_{1} \rightarrow \mathcal{L}_{2}$ be an isomorphism. Let $\gamma=\mathrm{C}(\varphi): F_{1} \rightarrow F_{2}$ which we extend to an isomorphism $\bar{\gamma}: \bar{F}_{1} \rightarrow \bar{F}_{2}$. Choose $\mathcal{T}_{1}$, $\mathcal{H}_{1}$ and $>$ for $\mathcal{L}_{1}$ as in 14.1.3(b), and use these to compute the index $\left(\Pi_{1}, \Pi_{10}, *\right)$ for $\mathcal{L}_{1}$. Next using $\varphi$ and $\bar{\gamma}$, we can transfer $\mathcal{T}_{1}, \mathcal{H}_{1}$ and $>$ to corresponding $\mathcal{T}_{2}$, $\mathcal{H}_{2}$ and $>$ for $\mathcal{L}_{2}$, and use these to compute $\left(\Pi_{2}, \Pi_{20}, *\right)$ for $\mathcal{L}_{2}$. (This is permitted by 14.1.1(b).) Then we can show that there exists a diagram isomorphism $\lambda$ : $\Pi_{1} \rightarrow \Pi_{2}$ and a group isomorphism $\nu: \Gamma_{1} \rightarrow \Gamma_{2}$ (conjugation by $\bar{\gamma}$ ) such that $\lambda\left(\sigma^{*} \alpha\right)=\nu(\sigma)^{*} \lambda(\alpha)$ for $\sigma \in \Gamma_{1}, \alpha \in \Pi_{1}$. This clearly implies similarity.

\subsection{The index of algebras in $\mathbb{M}_{2}$.}

14.2.1. If $\mathcal{L} \in \mathbb{M}_{n}$, then $\mathcal{L}$ is prime perfect and fgc by Proposition 4.4.3, and hence the index of $\mathcal{L}$ is defined.

Theorem 14.2.2. Two algebras in $\mathbb{M}_{2}$ that do not satisfy Condition (AA) are isomorphic if and only if they have similar indices.

Proof. The implication " $\Longrightarrow$ " follows from Lemma 14.1.5. The converse follows from 14.1.3(c) and Corollary 13.3.3.

14.2.3. Theorem 14.2 .2 provides a positive answer to Conjecture 6.4 in [GP1]. ${ }^{4}$

14.2.4. In rest of this section, we describe how to calculate the index of an algebra $\mathcal{L}$ in $\mathbb{M}_{2}$. We omit most of the details here, but rather sketch three examples that will be enough for the interested reader to complete the details.

We summarize our results in Table 4. In that table, column 1 lists our label from Table 3 for the algebra $\mathcal{L}$, and column 2 lists the index of $\mathcal{L}$. (Column 3 contains information that we will discuss in Section 14.4.) As before, when an entry in columns 2 or 3 depends on the parity of $k$, the first expression in the column applies when $k$ is even and the second when $k$ is odd.

Example 14.2.5. To give our first example, suppose that $\mathcal{L}=\mathrm{E}_{7}^{(1,2)}$. Then, $\mathcal{L}$ has absolute type $\mathrm{E}_{7}$ and relative type $\mathrm{F}_{4}$. But using the list of indices in Table 2 of $[\mathrm{T}]$ and the algorithm (see 14.1.3(c)) for calculating the relative type from the index, we find that there is only one possible index, namely $\mathrm{E}_{7,4}^{9}$, yielding these relative and absolute types. (In fact this is true over any field $F$.) So we conclude that $\mathcal{L}$ has index $\mathrm{E}_{7,4}^{9}$.

14.2.6. The same simple method (which with some practice goes quite quickly) works for most of the algebras in $\mathbb{M}_{2}$. The exceptions are the following algebras:

$$
\begin{gathered}
\mathrm{A}_{k}^{(1, \operatorname{rot}(q))}, k \geq 1,0 \leq q \leq\left\lfloor\frac{k+1}{2}\right\rfloor, \operatorname{gcd}(q, k+1)=1 ; \\
\mathrm{C}_{k}^{(1,2)}, k \text { odd }, k \geq 3 ; \quad \mathrm{D}_{k}^{(1,2 b)}, k \geq 4 ; \quad \mathrm{D}_{4}^{(1,3)} ; \quad \mathrm{D}_{k}^{(1,4)}, k \geq 4 ; \quad \text { and } \mathrm{E}_{6}^{(1,1)} .
\end{gathered}
$$

To handle each of these cases, we construct a matrix model for the algebra and then use the Descriptions in Table 2 of [T] (or a direct calculation) to identify the index.

\footnotetext{
${ }^{4}$ A partial proof of this conjecture for nullity two algebras of classical absolute type of "sufficiently large" rank was given by A. Steinmetz-Zikesch in [S-Z]. His proof is in fact rather explicit, and the algebras in question are described in full detail. Another proof of a more general version of the conjecture will appear in [GP2].
} 


\begin{tabular}{|c|c|c|}
\hline Label for $\mathcal{L}$ & Index & SEARS \\
\hline $\begin{array}{l}\mathrm{A}_{k}^{(1, \operatorname{rot}(q))}, k \geq 1 \\
0 \leq q \leq\left\lfloor\frac{k+1}{2}\right\rfloor\end{array}$ & $\begin{array}{c}{ }^{1} \mathrm{~A}_{k, r}^{\left(\frac{k+1}{r+1}\right)}, \\
r=\operatorname{gcd}(q, k+1)-1\end{array}$ & $\begin{array}{l}\mathrm{A}_{r}^{(1,1)} \\
\text { if } r \geq 1\end{array}$ \\
\hline $\mathrm{A}_{k}^{(1,2 a)}, k \geq 2$ & ${ }^{2} \mathrm{~A}_{k, \frac{k}{2}}^{(1)},{ }^{2} \mathrm{~A}_{k, \frac{k+1}{2}}^{(1)}$ & $\mathrm{BC}_{\frac{k}{2}}^{(2,1)}, \quad \mathrm{C}_{\frac{k+1}{2}}^{(1,2)}$ \\
\hline $\mathrm{A}_{k}^{(1,2 b)}, k$ odd $\geq 3$ & ${ }^{2} \mathrm{~A}_{k, \frac{k-1}{2}}^{(1)}$ & $\mathrm{BC}_{\frac{k-1}{2}}^{(2,2)}(2)$ \\
\hline $\mathrm{B}_{k}^{(1,1)}, k \geq 3$ & $\mathrm{~B}_{k, k}$ & $\mathrm{~B}_{k}^{(1,1)}$ \\
\hline $\mathrm{B}_{k}^{(1,2)}, k \geq 3$ & $\mathrm{~B}_{k, k-1}$ & $\mathrm{~B}_{k-1}^{(2,2) *}$ \\
\hline $\mathrm{C}_{k}^{(1,1)}, k \geq 2$ & $\mathrm{C}_{k, k}^{(1)}$ & $\mathrm{C}_{k}^{(1,1)}$ \\
\hline $\mathrm{C}_{k}^{(1,2)}, k \geq 2$ & $\mathrm{C}_{k, \frac{k}{2}}^{(2)}, \mathrm{C}_{k, \frac{k-1}{2}}^{(2)}$ & $\mathrm{C}_{\frac{k}{2}}^{(1,1) *,} \quad \mathrm{BC}_{\frac{k-1}{2}}^{(1,1)}$ \\
\hline $\mathrm{D}_{k}^{(1,1)}, k \geq 4$ & ${ }^{1} \mathrm{D}_{k, k}^{(1)}$ & $\mathrm{D}_{k}^{(1,1)}$ \\
\hline $\mathrm{D}_{k}^{(1,2 a)}, k \geq 4$ & ${ }^{2} \mathrm{D}_{k, k-1}^{(1)}$ & $\mathrm{B}_{k-1}^{(1,2)}$ \\
\hline $\mathrm{D}_{k}^{(1,2 b)}, k \geq 4$ & ${ }^{1} \mathrm{D}_{k, k-2}^{(1)}$ & $\mathrm{B}_{k-2}^{(2,2)}$ \\
\hline $\mathrm{D}_{k}^{(1,2 c)}, k \geq 5$ & ${ }^{1} \mathrm{D}_{k, \frac{k}{2}}^{(2)},{ }^{2} \mathrm{D}_{k, \frac{k-1}{2}}^{(2)}$ & $\mathrm{C}_{\frac{k}{2}}^{(2,2)}, \quad \mathrm{BC}_{\frac{k-1}{2}}^{(2,2)}(1)$ \\
\hline $\mathrm{D}_{4}^{(1,3)}$ & ${ }^{3} \mathrm{D}_{4,2}^{2}$ & $\mathrm{G}_{2}^{(1,3)}$ \\
\hline $\mathrm{D}_{k}^{(1,4)}, k \geq 4$ & ${ }^{2} \mathrm{D}_{k, \frac{k-2}{2}}^{(2)},{ }^{1} \mathrm{D}_{k, \frac{k-3}{2}}^{(2)}$ & $\mathrm{BC}_{\frac{k-2}{2}}^{(2,4)}, \quad \mathrm{BC}_{\frac{k-3}{2}}^{(4,4)}$ \\
\hline $\mathrm{E}_{6}^{(1,1)}$ & ${ }^{1} \mathrm{E}_{6,6}^{0}$ & $\mathrm{E}_{6}^{(1,1)}$ \\
\hline $\mathrm{E}_{6}^{(1,2)}$ & ${ }^{2} \mathrm{E}_{6,4}^{2}$ & $\mathrm{~F}_{4}^{(1,2)}$ \\
\hline $\mathrm{E}_{6}^{(1,3)}$ & ${ }^{1} \mathrm{E}_{6,2}^{16}$ & $\mathrm{G}_{2}^{(3,3)}$ \\
\hline $\mathrm{E}_{7}^{(1,1)}$ & $\mathrm{E}_{7,7}^{0}$ & $\mathrm{E}_{7}^{(1,1)}$ \\
\hline $\mathrm{E}_{7}^{(1,2)}$ & $\mathrm{E}_{7,4}^{9}$ & $\mathrm{~F}_{4}^{(2,2)}$ \\
\hline $\mathrm{E}_{8}^{(1,1)}$ & $\mathrm{E}_{8,8}^{0}$ & $\mathrm{E}_{8}^{(1,1)}$ \\
\hline $\mathrm{F}_{4}^{(1,1)}$ & $\mathrm{F}_{4,4}^{0}$ & $\mathrm{~F}_{4}^{(1,1)}$ \\
\hline $\mathrm{G}_{2}^{(1,1)}$ & $\mathrm{G}_{2,2}^{0}$ & $\mathrm{G}_{2}^{(1,1)}$ \\
\hline
\end{tabular}

TABLE 4. The index and the SEARS of $\mathcal{L} \in \mathbb{M}_{2}$

Example 14.2.7. Suppose that $\mathcal{L}=\mathrm{A}_{k}^{(1, \operatorname{rot}(q))}$, with $k$ and $q$ as in (46). Then, by (45), we can identify $\mathcal{L}=\mathfrak{s l}_{1}(Q(\theta))$, where $\theta$ has order $m=k+1$ in $\mathbb{k}^{\times}$. Then, we identify the centroid of $\mathcal{L}$ with $R_{2}$ as in Lemma 4.5.6(e); and, since $K_{2} / R_{2}$ is flat, we have $\widetilde{\mathcal{L}}=\mathfrak{s l}_{1}(Q(\theta)) \otimes_{R_{2}} K_{2} \simeq \mathfrak{s l}_{1}\left(Q(\theta) \otimes_{R_{2}} K_{2}\right)$. But, by Lemma 11.2.2, $Q(\theta) \otimes_{R_{2}} K_{2}$ is a central division algebra of degree $k+1$ over $K_{2}$. Thus, by Table 2 of $[\mathrm{T}]$ (see the first Description on p. 55 of $[\mathrm{T}]$ ), $\mathcal{L}$ has index ${ }^{1} \mathrm{~A}_{k, 0}^{(k+1)}$.

We are left with the algebras in (47). For these we use the easy part (the construction) in the coordinatization theorems for centerless Lie tori (see 5.3.6).

Example 14.2.8. Suppose that $\mathcal{L}=\mathrm{D}_{k}^{(1,4)}$ with $k \geq 4$ and $k$ even. Then, $\mathcal{L}$ has relative type $\mathrm{BC}_{\frac{k-1}{2}}$. To describe a construction of $\mathcal{L}$, let $\mathcal{A}=Q(-1)$, the unital associative algebra presented by the generators $x_{1}, x_{2}, x_{1}^{-1}, x_{2}^{-1}$ subject to 
the inverse relations and the relation $x_{1} x_{2}=-x_{2} x_{1}$. We identify the centre of $\mathcal{A}$ with $R_{2}$ as in 4.5.3 with $m=2$. Let $*$ be the $R_{2}$-linear involution on $\mathcal{A}$ such that $x_{i}^{*}=x_{i}$ for $i=1,2$. Let $J_{k-2}$ be the matrix in $\mathrm{M}_{k-2}(\mathcal{A})$ whose $(i, j)$ entry is $\delta_{i, k-1-j}$, and let $G=\operatorname{diag}\left(J_{k-2}, 1, x_{1}\right)$ (in block diagonal form) in $\mathcal{E}=\mathrm{M}_{k}(\mathcal{A})$. Note that $G$ is a unit in the $R_{2}$-algebra $\mathcal{E}$ and $\left(G^{*}\right)^{t}=G$, so we may define an $R_{2}$-linear involution $\tau$ on $\mathcal{E}$ by $\tau(T)=G^{-1}\left(T^{*}\right)^{t} G$. We set

$$
\mathcal{S}=\{T \in \mathcal{E} \mid \tau(T)=-T\},
$$

in which case $\mathcal{S}$ is a Lie algebra over $R_{2}$ under the commutator product.

We chose to look at $\mathcal{S}$ since it arises in [AB, Theorem 6.3.1], which is the coordinatization theorem for centreless $(\Lambda, \Delta)$-tori for $\Delta$ of type $\mathrm{BC}_{r}$, where in our case $r=\frac{k-1}{2}$. Indeed the first part of that theorem tells us that $\mathcal{S}$ is a centreless Lie $(\Lambda, \Delta)$-torus, where $\Lambda$ has rank 2 and $\Delta$ is of type $\mathrm{BC}_{\frac{k-1}{2}}$. (See also 7.2.3 of $[\mathrm{AB}]$.) Moreover, since $*$ is nontrivial, $\mathcal{S}$ has full root support [AB, Remark 8.1.1]. Also, it follows from Propositions 4.10 and 6.21 of $[\mathrm{AB}]$ that the centroid of $\mathcal{S}$ is identified with $R_{2}$ using the natural action of $R_{2}$ on $\mathcal{S}$. Since it is clear that $\mathcal{S}$ is finitely generated as an $R_{2}$-module, $\mathcal{S}$ is fgc. Hence, by Theorem 5.5.1, $\mathcal{S} \in \mathbb{M}_{2}$ and the relative type of $\mathcal{S}$ is $\mathrm{BC}_{\frac{k-1}{2}}$.

We next look at the central closure of $\mathcal{S}$. For this we set $\widetilde{\mathcal{A}}=\mathcal{A} \otimes_{R_{2}} K_{2}$, $\widetilde{\mathcal{E}}=\mathcal{E} \otimes_{R_{2}} K_{2}$, and $\widetilde{\mathcal{S}}=\mathcal{S} \otimes_{R_{2}} K_{2}$; and we extend $*$ and $\tau$ to $K_{2}$-linear involutions of $\widetilde{\mathcal{A}}$ and $\widetilde{\mathcal{E}}$ respectively. One checks that

$$
\widetilde{\mathcal{S}}=\{T \in \widetilde{\mathcal{E}} \mid \tau(T)=-T\} .
$$

Further, we know from Lemma 11.2.2 that $\mathcal{A}$ is a central division algebra of degree 2 over $K_{2}$; and its clear that $*$ is an orthogonal involution on $\mathcal{A}$. Thus, by $[\mathrm{J}$, Theorem X.9], the absolute type of $\mathcal{S}$ is $\mathrm{D}_{k}$. Since we have matched relative and absolute types, we see that $\mathcal{L} \simeq \mathcal{S}$.

Finally from the information in the previous paragraph and from Table 2 of $[\mathrm{T}]$ (see the Descriptions on p. 57 of $[\mathrm{T}]$ ), it follows that $\mathcal{S}$ has index ${ }^{t} \mathrm{D}_{k, \frac{k-1}{2}}^{(2)}$, where $t=1$ or 2 . Moreover, $t=1$ if and only if the discriminant $\operatorname{disc}(\widetilde{\mathcal{E}}, \tau)$ of the algebra with involution $(\widetilde{\mathcal{E}}, \tau)$ over $K_{2}$ is trivial in $K_{2}^{\times} /\left(K_{2}^{\times}\right)^{2}$. Finally one calculates using $\left[\right.$ KMRT, 7.2] that $\operatorname{disc}(\widetilde{\mathcal{E}}, \tau)=-t_{1}^{k+1} t_{2}^{k}\left(K_{2}^{\times}\right)^{2} \neq 1\left(K_{2}^{\times}\right)^{2}$, since $k$ is even. So $\mathcal{L} \simeq \mathcal{S}$ has index ${ }^{2} \mathrm{D}_{k, \frac{k-1}{2}}^{(2)}$.

14.2.9. The remaining cases in (47) can be handled in a similar fashion. For the algebras $\mathrm{C}_{k}^{(1,2)}\left(k\right.$ odd), $\mathrm{D}_{k}^{(1,2 b)}$, and $\mathrm{D}_{k}^{(1,4)}(k$ odd), one again uses the construction from $\left[\mathrm{AB}\right.$, Theorem 6.3.1]. (That theorem covers quotient types $\mathrm{BC}_{r}$ and $\mathrm{B}_{r}$.) Finally for the algebras $\mathrm{D}_{4}^{(1,3)}$ and $\mathrm{E}_{6}^{(1,3)}$, one uses the construction from [AG, Thm. 5.63] which covers quotient type $\mathrm{G}_{2}{ }^{5}$

\subsection{Saito's extended affine root systems.}

In this section and the next, we assume for convenience that $\mathbb{k}=\mathbb{C}$, although it is very likely that with minor modifications the results mentioned carry over to the general case.

14.3.1. Let $V_{\mathbb{R}}$ be a finite dimensional real vector space with positive definite real valued symmetric bilinear form $(\mid)$. For $\alpha \in V_{\mathbb{R}}$ with $(\alpha, \alpha) \neq 0$, let $r_{\alpha}$ be the

\footnotetext{
${ }^{5}$ A different "cohomological" approach to computing the index will be described in [GP2].
} 
orthogonal reflection along $\alpha$. We will call a subset $\Sigma$ of $V_{\mathbb{R}}$ a Saito extended affine root system, or SEARS for short, if the following conditions hold: the natural map $\operatorname{span}_{\mathbb{Z}}(\Sigma) \otimes_{\mathbb{Z}} \mathbb{R} \rightarrow V_{\mathbb{R}}$ is bijective, $(\alpha, \alpha) \neq 0$ for $\alpha \in \Sigma, r_{\alpha}(\Sigma)=\Sigma$ for $\alpha \in \Sigma$, $2 \frac{(\alpha, \beta)}{(\alpha, \alpha)} \in \mathbb{Z}$ for $\alpha, \beta \in \Sigma$, and $\Sigma$ is irreducible in the usual sense. In that case, we say that $\Sigma$ is reduced if $\alpha \in \Sigma$ implies $2 \alpha \notin \Sigma$, and we define the null dimension of $\Sigma$ to be the the dimension of the radical of $V_{\mathbb{R}}$. If $\Sigma$ is a SEARS, then the image of $\Sigma$ in $V_{\mathbb{R}}$ modulo the radical of $(\mid)$ is the set of nonzero roots of an irreducible finite root system called the finite quotient root system of $\Sigma$ [Sai, Example 1.3 and Assertion 1.8]. Two SEARS $\Sigma$ in $V_{\mathbb{R}}$ and $\Sigma^{\prime}$ in $V_{\mathbb{R}}^{\prime}$ are said to be isomorphic if there is a vector space isomorphism from $V_{\mathbb{R}}$ onto $V_{\mathbb{R}}^{\prime}$ which maps $\Sigma$ onto $\Sigma^{\prime}$, in which case the forms are preserved up to nonzero scalar by the isomorphism [Sai, Lemma 1.4].

14.3.2. SEARS of null dimension $n$ were introduced by Saito in [Sai, §1.3], where they were called $n$-extended affine root systems. His motivation came from the study of elliptic singularities of complex analytic surfaces.

14.3.3. SEARS also play an important role in the theory of EALAs, because the set of nonisotropic roots $\Phi^{\times}$of a discrete EALA $(\mathfrak{g},(\mid), \mathfrak{h})$ of nullity $n$ is a reduced SEARS of null dimension $n$ (by [AABGP, Thm. 2.16] and [Az, Lemma 1.4]). Here $V_{\mathbb{R}}$ is the real span of $\Phi^{\times}$with symmetric form ( | ) induced and suitably normalized from the given form on $\mathcal{E}$.

14.3.4. (Classification of SEARS) In [Sai, §'s 5.2 and 5.4], Saito classified up to isomorphism all reduced SEARS of null dimension 2 that satisfy an additional condition, the existence of a marking $G$ such that $\Sigma / G$ is reduced. In [Az], Azam completed the classification of reduced SEARS of null dimension 2 by establishing a relationship between reduced SEARS and the root systems studied in [AABGP, Chap. 2]. He showed that, in addition to the root systems in Saito's classification, there are two infinite families that we will denote here by $\mathrm{BC}_{\ell}^{(1,1)}, \ell \geq 1$, and $\mathrm{BC}_{\ell}^{(4,4)}, \ell \geq 1$, following the notational conventions in [Sai]. They are

$$
\begin{gathered}
\mathrm{BC}_{\ell}^{(1,1)}=\left(\Delta_{\mathrm{sh}}+\Lambda\right) \cup\left(\Delta_{\mathrm{lg}}+\Lambda\right) \cup\left(\Delta_{\mathrm{ex}}+\Lambda \backslash(2 \Lambda)\right) \quad \text { and } \\
\mathrm{BC}_{\ell}^{(4,4)}=\left(\Delta_{\mathrm{sh}}+\Lambda \backslash(2 \Lambda+a)\right) \cup\left(\Delta_{\mathrm{lg}}+2 \Lambda\right) \cup\left(\Delta_{\mathrm{ex}}+4 \Lambda+2 a\right),
\end{gathered}
$$

where $\Delta_{\mathrm{sh}}, \Delta_{\mathrm{lg}}$ and $\Delta_{\mathrm{ex}}$ are the sets of roots of length 1,2 and 4 respectively in the irreducible finite root system of type $\mathrm{BC}_{\ell}, a, b$ is a basis for the radical of $(\mid)$, and $\Lambda=\mathbb{Z} a \oplus \mathbb{Z} b$. Note that the second term in each of these unions is empty if $\ell=1$. The complete nonredundant list of reduced SEARS of null dimension 2 up to isomorphism is then

$$
\begin{gathered}
\mathrm{A}_{\ell}^{(1,1)}(\ell \geq 1), \quad \mathrm{A}_{1}^{(1,1) *}, \\
\mathrm{~B}_{\ell}^{(1,1)}(\ell \geq 3), \quad \mathrm{B}_{\ell}^{(1,2)}(\ell \geq 3), \quad \mathrm{B}_{\ell}^{(2,2)}(\ell \geq 2), \quad \mathrm{B}_{\ell}^{(2,2) *}(\ell \geq 2), \\
\mathrm{C}_{\ell}^{(1,1)}(\ell \geq 2), \quad \mathrm{C}_{\ell}^{(1,2)}(\ell \geq 2), \quad \mathrm{C}_{\ell}^{(2,2)}(\ell \geq 3), \quad \mathrm{C}_{\ell}^{(1,1) *}(\ell \geq 2), \\
\mathrm{BC}_{\ell}^{(2,1)}(\ell \geq 1), \quad \mathrm{BC}_{\ell}^{(2,4)}(\ell \geq 1), \quad \mathrm{BC}_{\ell}^{(2,2)}(1)(\ell \geq 2), \quad \mathrm{BC}_{\ell}^{(2,2)}(2)(\ell \geq 1), \\
\mathrm{BC}_{\ell}^{(1,1)}(\ell \geq 1), \quad \mathrm{BC}_{\ell}^{(4,4)}(\ell \geq 1), \\
\mathrm{D}_{\ell}^{(1,1)}(\ell \geq 4), \quad \mathrm{E}_{\ell}^{(1,1)}(\ell=6,7,8), \\
\mathrm{F}_{4}^{(1,1)}, \quad \mathrm{F}_{4}^{(1,2)}, \quad \mathrm{F}_{4}^{(2,2)}, \quad \mathrm{G}_{2}^{(1,1)}, \quad \mathrm{G}_{2}^{(1,3)} .
\end{gathered}
$$


(See [Sai, §'s 5.2] and (48) above for the definitions of these root systems.) Note that the type of the finite quotient root system of a reduced SEARS $\Sigma$ of null dimension 2 is used as the "base" in the notation for $\Sigma$. For example, the reduced SEARS of null dimension 2 with finite quotient root system of type $\mathrm{BC}_{\ell}$ occur in Rows 4 and 5 of (49).

\subsection{The SEARS of an isotropic algebra in $\mathbb{M}_{2}$.}

Suppose that $\mathbb{k}=\mathbb{C}$ and that the primitive roots of unity are chosen as $\zeta_{m}=$ $e^{\frac{2 \pi \sqrt{-1}}{m}}$ for $m \geq 1$. Let $\mathcal{L}$ be an isotropic algebra in $\mathbb{M}_{2}$.

14.4.1. By Theorem 13.3.1, there exists a unique affine GCM $A$ and a unique $\sigma \in \operatorname{Aut}(A)$ such that $(A, \sigma)$ appears in columns 1 and 2 of Table 2 and $\mathcal{L} \simeq \mathrm{L}\left(\overline{\mathfrak{g}^{\prime}}, \sigma\right)$, where $\mathfrak{g}=\mathfrak{g}(A)$. We note that the assumption that $\mathcal{L}$ is anisotropic precisely rules out the case in Row 1 of Table 2 where $\operatorname{gcd}(q, k+1)=1$. We set $\mathfrak{g}=\mathfrak{g}(A)$ and use the above isomorphism to identify

$$
\mathcal{L}=\mathrm{L}_{m}\left(\overline{\mathfrak{g}^{\prime}}, \sigma\right),
$$

where $m$ is the order of $\sigma$. Since $\sigma$ is not transitive, we can follow 9.1.1 and construct a discrete EALA

$$
\mathcal{E}=\mathrm{L}_{m}(\mathfrak{g}, \sigma) \oplus \mathbb{k} \tilde{c} \oplus \mathbb{k} \tilde{d},
$$

with nondegenerate symmetric bilinear form $(\mid)$ and ad-diagonalizable abelian subalgebra $\mathcal{H}=\mathfrak{h}^{\sigma} \oplus \mathbb{k} \tilde{c} \oplus \mathbb{k} \tilde{d}$. Then, by Proposition 9.1.3, $\mathcal{E}$ is a discrete EALA of nullity 2 with centreless core isomorphic to $\mathcal{L}$. Let $\Phi$ be the root system for $\mathcal{E}$ relative to $\mathcal{H}$. By 14.3.3, $\Phi^{\times}$is a reduced SEARS of null dimension 2 which we call the $S E A R S$ of $\mathcal{L}$.

Remark 14.4.2. The SEARS of $\mathcal{L}$ just defined depends only on the isomorphism class of $\mathcal{L}$ because of the uniqueness of $A$ and $\sigma$ in the above discussion. Indeed, once these have been selected, $\mathcal{E}, \mathcal{H}$ and $(\mid)$ are defined in terms of $A$ and $\sigma$. It would be desirable to have a more intrinsic proof of the invariance of the SEARS of $\mathcal{L}$.

Proposition 14.4.3. Let $\Phi^{\times}$be the $S E A R S$ of $\mathcal{L}$, and let $X_{\ell}$ be the type of the finite quotient root system of $\Phi^{\times}$. Then, $\mathcal{L}$ has relative type $X_{\ell}$.

Proof. Let $\mathcal{E}$ be the EALA described in described in 14.4.1. It follows from Remark 5.1.6 that $X_{\ell}$ is the quotient type of $\mathcal{E}$. But, by Proposition 9.1.3, we have $\mathcal{E}_{c c} \simeq \mathcal{L}$, so our conclusion follows from Corollary 5.5.2(a).

14.4.4. We now use the work of U. Pollmann to list, in Column 3 of Table 4, the SEARS of each isotropic $\mathcal{L}$ in $\mathbb{M}_{2}$.

Indeed Pollmann did most of the work. For each algebra $\mathrm{L}\left(\overline{\mathfrak{g}^{\prime}}, \sigma\right)$ in Table 2, except those in Row 1 , she calculated the root system $\Phi$ of the EALA $\mathcal{E}$ described in 14.4.1. (She actually worked with a central quotient of $\mathcal{E}$ rather than $\mathcal{E}$, but this does not change the root system.) In view of Lemma 5.1.7(b), this determines $\Phi^{\times}$as well.

This leaves us with the case when $\mathcal{L}=\mathrm{A}_{k}^{(1, \operatorname{rot}(q))}$, where $k \geq 1,0 \leq q \leq\left\lfloor\frac{k+1}{2}\right\rfloor$, and $r:=\operatorname{gcd}(q, k+1)-1 \geq 1$. Then, by Table $2, \mathcal{L}$ has relative type $\mathrm{A}_{r}$. Now, if $r \geq 2$, there is only one reduced SEARS of null dimension 2 with finite quotient root system $\mathrm{A}_{r}$, so by Proposition 14.4.3, $\Phi^{\times} \simeq \mathrm{A}_{r}^{(1,1)}$. It remains to consider the case when $r=1$. In this case, the root system $\Phi$ (and hence $\Phi^{\times}$) can be found by 
direct calculation using the realization $(45)$ of $\mathcal{L}$ in terms of a quantum torus, and we obtain $\Phi^{\times} \simeq \mathrm{A}_{1}^{(1,1)}$. The details of this calculation are somewhat delicate but straightforward, and we leave them to the reader.

Remark 14.4.5. In Row 7 , Column 3 of Table $4, \mathrm{C}_{\frac{k}{2}}^{(1,1) *}$ should be interpreted as $\mathrm{A}_{1}^{(1,1) *}$ when $k=2$.

A comparison of Column 3 of Table 4 with the list (49) now yields our final result.

Theorem 14.4.6. Every reduced SEARS of null dimension 2 arises as the SEARS of some isotropic algebra in $\mathbb{M}_{2}$. Moreover, if $\mathcal{L}$ and $\mathcal{L}^{\prime}$ are algebras in $\mathbb{M}_{2}$ that do not satisfy Condition $(A A)$ (and in particular are isotropic), then $\mathcal{L}$ and $\mathcal{L}^{\prime}$ are isomorphic if and only if their SEARS are isomorphic.

\section{REFERENCES}

[A] B. Allison, Some isomorphism invariants for Lie tori, in preparation.

[AB] B Allison and G. Benkart, Unitary Lie algebras and Lie tori of type $B C_{r}, r \geq 3$, in Proceedings of the Conference on Quantum Affine Algebras, Extended Affine Lie Algebras and Applications, Banff, Canada, 2008, Contemporary Mathematics 506 (2010), 1-47, Amer. Math Soc., Providence, RI.

[AABGP] B. Allison, S. Azam, S. Berman, Y. Gao and A. Pianzola, Extended affine Lie algebras and their root systems, Mem. Amer. Math. Soc. 126 (603), 1997.

[ABFP1] B. Allison, S. Berman, J. Faulkner, A. Pianzola, Realization of graded-simple algebras as loop algebras, Forum Mathematicum, 20 (2008), 395-432

[ABFP2] B. Allison, S. Berman, J. Faulkner, A. Pianzola, Multiloop realization of extended affine Lie algebras and Lie tori, Trans. Amer. Math. Soc. 361 (2009), 4807-4842.

[ABGP] B. Allison, S. Berman, Y. Gao and A. Pianzola, A characterization of affine Kac-Moody Lie algebras, Comm. Math. Phys. 185 (1997), 671-688.

[ABP1] B. Allison, S. Berman and A. Pianzola, Covering algebras I: Extended affine Lie algebras, J. Algebra 250 (2002), 485-516.

[ABP2] B. Allison, S. Berman and A. Pianzola, Covering algebras II: Isomorphism of loop algebras, J. Reine Angew. Math. 571 (2004), 39-71.

[ABP2.5] B. Allison, S. Berman and A. Pianzola, Iterated loop algebras, Pacific J. Math. 227 (2006), 1-42.

[ABPOb] B. Allison, S. Berman and A. Pianzola, Multiloop algebras and extended affine Lie algebras of nullity 2, Oberwolfach Report 55 (2006), 3280-3283.

[AF] B. Allison and J. Faulkner, Isotopy for extended affine Lie algebras and Lie tori, arXiv:math/0709.1181v2.

[AG] B. Allison and Y. Gao, The root system and the core of an extended affine Lie algebra, Selecta Math. (N.S.) 7 (2001), no. 2, 149-212.

[Az] S. Azam, Extended affine root systems, J. Lie Theory 12 (2002), 515-527.

[Bau] J. Bausch, Étude et classification des automorphismes d'ordre fini et de première espèce des algèbres de Kac-Moody affines, in Algèbres de Kac-Moody affines, 5-124, Univ. Nancy, Nancy.

[BN] G. Benkart and E. Neher, The centroid of extended affine and root graded Lie algebras, J. Pure Appl. Algebra 205 (2006), no. 1, 117-145.

[BGK] S. Berman, Y. Gao and Y. Krylyuk, Quantum tori and the structure of elliptic quasisimple Lie algebras, J. Funct. Anal. 135 (1996), no. 2, 339-389.

[Bo1] N. Bourbaki, Commutative algebra. Chapters 1-7, Translated from the French. Reprint of the 1972 edition. Elements of Mathematics (Berlin). Springer-Verlag, Berlin, 1989.

[Bo2] N. Bourbaki, Lie groups and Lie algebras. Chapters 4-6, Translated from the French, Elements of Mathematics, Springer-Verlag, Berlin, 2002, MR1890629 (2003a:17001).

[D] P.K. Draxl, Skew fields, Cambridge Univ. Press, Cambridge, 1983.

[EMY] S. Eswara Rao, R. Moody and T. Yokonuma, Toroidal Lie algebras and vertex representations, Geom. Ded. 35 (1990), 283-307. 
[FSS] J. Fuchs, B. Schellekens and C. Schweigert, From Dynkin diagram symmetries to fixed point structures, Commun. Math. Phys. 180 (1996), 39-97.

[GP1] P. Gille and A. Pianzola, Galois cohomology and forms of algebras over Laurent polynomial rings, Math. Ann. 338 (2007), 497-543.

[GP2] P. Gille and A. Pianzola, Galois cohomology and forms of algebras over Laurent polynomial rings $I I$, in preparation.

[H-KT] Høegh-Krohn and B. Torresani, Classification and construction of quasi-simple Lie algebras, J. Funct. Anal. 89 (1990), 106-136.

[J] N. Jacobson, Lie algebras, Dover, New York, 1979.

[K1] V. Kac, Simple irreducible graded Lie algebras of finite growth, Izv. Akad. Nauk SSSR Ser. Mat. 32 (1968), 1323-1367. English translation: Math. USSR-Izvestija 2 (1968), $1271-1311$

[K2] V. Kac, Infinite dimensional Lie algebras, third edition, Cambridge University Press, Cambridge, 1990.

[KMRT] M.-A. Knus, A. Merkurjev, M. Rost and J.-P. Tignol, The book of involutions, Amer. Math. Soc. Colloquium Publications, 44, Amer. Math. Soc., Providence, RI, 1998.

$[\mathrm{KW}] \quad$ V. Kac and S.P. Wang, On automorphisms of Kac-Moody algebras and groups, Advances in Math. 92 (1992), 129-195.

[LN] O. Loos and E. Neher, Reflection systems and partial root systems, Jordan theory preprint archives, http://homepage.uibk.ac.at/ c70202/jordan/, 2005.

[Ma] Y.I. Manin, Topics in Noncommutative Geometry, Princeton University Press, 1991.

[Mi] J.S. Milne, Étale cohomology, Princeton University Press, 1980.

[Mo] R.V. Moody, Euclidean Lie algebras, Canad. J. Math. 21 (1969), 1432-1454.

[MP] R.V. Moody and A. Pianzola, Lie algebras with triangular decomposition, John Wiley, New York, 1995.

[Na] K. Naoi, Multiloop Lie algebras and the construction of extended affine Lie algebras, preprint, arXiv:0807.2019v1.

[Neeb] K.-H. Neeb, On the classification of rational quantum tori and the structure of their automorphism groups, Canad. Math. Bull. 51 (2008), 261-282.

[Neh1] E. Neher, Lie tori, C. R. Math. Acad. Sci. Soc. R. Can. 26 (2004), no. 3, 84-89.

[Neh2] E. Neher, Extended affine Lie algebras, C. R. Math. Acad. Sci. Soc. R. Can. 26 (2004), no. 3, 90-96.

[Neh3] E. Neher, Extended affine Lie algebras and other generalizations of affine Lie algebras - a survey, arXiv:0805.3460.

[P1] A. Pianzola, Affine Kac-Moody Lie algebras and torsors over the punctured line, Indag. Mathem., N.S. 13 (2002), 249-257.

[P2] A. Pianzola, Vanishing of $H^{1}$ for Dedekind rings and applications to loop algebras, C.R. Acad. Sci. Paris, Ser I 340 (2005), 633-638.

[PK] D.H. Peterson and V. Kac, Infinite flag varieties and conjugacy theorems, Proc. Natl. Acad. Sci. USA 80 (1983), 1778-1782.

[Pi] R. Pierce, Associative algebras, Springer-Verlag, Berlin, 1982.

[Po] U. Pollmann, Realisation der biaffinen Wurzelsysteme von Saito in Lie-Algebren, Hamburger Beiträge zur Mathematik aus dem Mathematischen Seminar, Heft 29 (1994), $1-105$.

[Sai] K. Saito, Extended affine root systems 1 (Coxeter transformations, Publ. RIMS., Kyoto Univ. 21 (1985), 75-179.

[Sat] I Satake, On the theory of reductive algebraic groups over a perfect field, J. Math. Soc. Japan 15 (1963), 210-235.

[Se1] G.B. Seligman, Topics in Lie Algebras, Mimeographed notes, Yale University, New Haven, Conn., 1969.

[Se2] G.B. Seligman, Rational methods in Lie algebras, Lect. Notes in Pure and Applied Math. 27, Marcel Dekker, New York, 1976.

[S-Z] A. Steinmetz-Zikesch, Algèbres de Lie de dimension infinie et théorie de la descente, Thèse, Université Paris-Sud, 2009.

[T] J. Tits, Classification of algebraic semisimple groups, Algebraic Groups and Discontinuous Subgroups (Proc. Sympos. Pure Math., Boulder, Colo., 1965), pp. 33-62, Amer. Math. Soc., Providence, R.I., 1966.

[vdL] J. van de Leur, Twisted toroidal Lie algebras, preprint, arXiv math/0106119 v1, 2001. 
[W] M. Wakimoto, Extended affine Lie algebras and a certain series of Hermitian representations, preprint (1985).

[Y1] Y. Yoshii, Coordinate algebras of extended affine Lie algebras of type $\mathrm{A}_{1}$, J. Algebra 234 (2000), 128-168.

[Y2] Y. Yoshii, Lie tori-A simple characterization of extended affine Lie algebras, Publ. Res. Inst. Math. Sci. 42 (2006), no. 3, 739-762.

(Bruce Allison) Department of Mathematics and Statistics, University of Victoria, PO BOX 3060 STN CSC, Victoria BC CANADA V8W 3R4

E-mail address: ballison@uvic.ca

(Stephen Berman) Saskatoon, Saskatchewan, Canada

E-mail address: sberman@shaw.ca

(Arturo Pianzola) Department of Mathematical and Statistical Sciences, University of Alberta, Edmonton, Alberta, Canada T6G 2G1

(Arturo Pianzola) Instituto Argentino de Matemtica, SaAvedra 15, 1083 Buenos Aires, Argentina

E-mail address: a.pianzola@ualberta.ca 